\title{
Targeted lentiviral vectors pseudotyped with the Tupaia paramyxovirus glycoproteins
}

\author{
Dissertation
}

submitted to the

Combined Faculties for the Natural Sciences and for Mathematics of the Ruperto-Carola University of Heidelberg, Germany for the degree of Doctor of Natural Sciences

presented by

Diplom-Biologin Theresa Enkirch born in Hadamar, Germany 



\title{
Dissertation
}

submitted to the

Combined Faculties for the Natural Sciences and for Mathematics

of the Ruperto-Carola University of Heidelberg, Germany

for the degree of

Doctor of Natural Sciences

\author{
presented by \\ Diplom-Biologin Theresa Enkirch \\ born in Hadamar, Germany
}

Oral examination: 
Targeted lentiviral vectors pseudotyped with the Tupaia paramyxovirus glycoproteins 



\section{DECLARATION BY THE CANDIDATE}

I hereby declare that this thesis is my own work and effort. Where other sources of information have been used, they have been indicated or acknowledged.

Signature:

Date: 02.11 .2011 


\section{Acknowledgement}

I would like to express my gratitude to all those who supported me during my thesis.

First acknowledgement goes to my supervisor Dr. Dr. Christoph Springfeld for the opportunity to work on these projects, for his guidance, his scientific encouragement and the opportunity to perform my $\mathrm{PhD}$ thesis in his research group.

I would also thank Prof. Dr. Bartenschlager for taking the task of the first reviewer of my PhD thesis and for the review of this work. Special thanks go to Guy, Sascha, Chris, Mathias, Martin, Karim, Christine and Jessica from the group 'Viro-Therapy' for their advice and scientific discussions. I would also like to thank Petra, Iris, Sabine, Dieter, Yvonne and Solveigh for the warm working atmosphere as well as the fun we had during the time we spent together. An extra special thank you goes to Birgit Hoyler for all the work she put into my project, the great help she gave me during the last few months of my $\mathrm{PhD}$ and for being such a great lab mate. Without her everything would have taken so much longer!!! I am also grateful to Prof. Dr. Valerie Bosch who was member of my thesis advisory committee. Many thanks go to all members of the lab of Michael Pawlita, for permanent support and great times at lab excursions and outside the lab.

I am incredibly grateful to all members of the section 6/5 Viral Gene Transfer Medicinal Products' of the Paul-Ehrlich-Institut, Anke, Sabrina, Patricia, Irene, Robert, Thorsten, Sarah, Iris, Qi, Tobi, Katharina, Julia, Gundi and Manu. I really enjoyed working with them and the time we spend together outside the lab! Especially, I would like to thank Prof. Dr. Christian J. Buchholz for taking the task of the second reviewer, for welcoming me into his lab and giving me his inspiration and support throughout my project.

I thank Prof. Suat Özbek and Dr. Freddy Frischknecht for reading and evaluating my thesis.

Many thanks go to my friends Allan, Vanessa, Anna und Bernd for all their support! Finally I would like to thank my parents, Marita und Manfred and my brother Tobias for supporting me during the last years.

You were all a great help! 
Parts of the presented work have been published:

Enkirch T., Kneissl S., Hoyler B., Stremmel W., Buchholz C.J., Springfeld C.: Development and characterization of targeted lentiviral vectors pseudotyped with the Tupaia paramyxovirus glycoproteins; $4^{\text {th }}$ European Congress of Virology, Cernobbio, Italy, April 2010; oral presentation

Enkirch T., Kneissl S., Hoyler B., Stremmel W., Buchholz C.J., Springfeld C.: Development and characterization of targeted lentiviral vectors pseudotyped with the Tupaia paramyxovirus glycoproteins; $21^{\text {st }}$ Annual Meeting of the Society for Virology, Freiburg, Germany, March 2011; Poster presentation

Enkirch T., Kneissl S., Hoyler B., Stremmel W., Buchholz C.J., Springfeld C.: Development and characterization of targeted lentiviral vectors pseudotyped with the Tupaia paramyxovirus glycoproteins; $14^{\text {th }}$ Annual Meeting of the American Society of Gene an Cell Therapy, Seattle, WA, USA, May 2011; Poster presentation

T. Enkirch, S. Kneissl, B. Hoyler, G. Ungerechts, W. Stremmel, C. J. Buchholz, C. Springfeld: Targeted lentiviral vectors pseudotyped with the Tupaia paramyxovirus glycoproteins; Gene Therapy; Manuscript in revision 


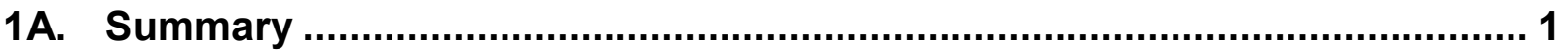

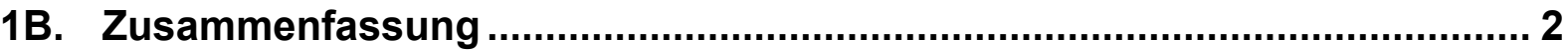

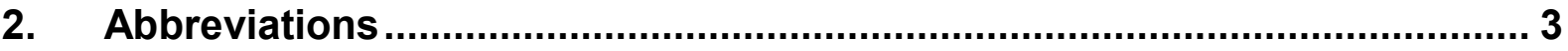

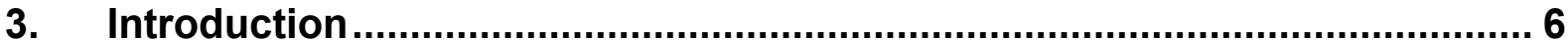

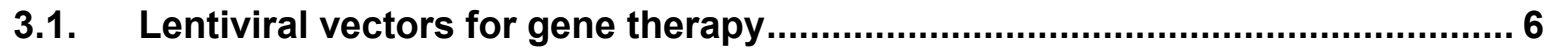

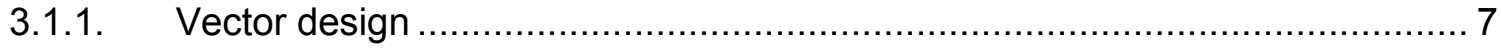

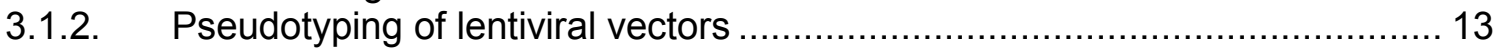

3.1.3. Targeting strategies for lentiviral vectors ............................................ 15

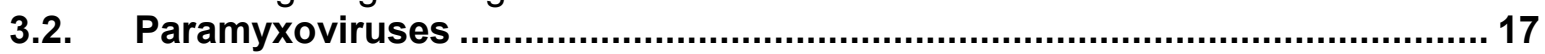

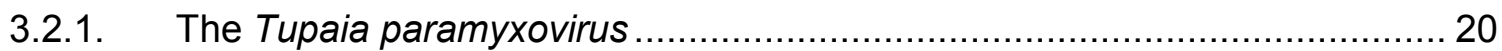

3.2.2. Glycoproteins of the Tupaia paramyxovirus.......................................... 22

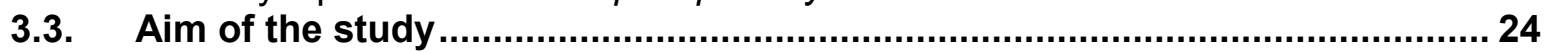

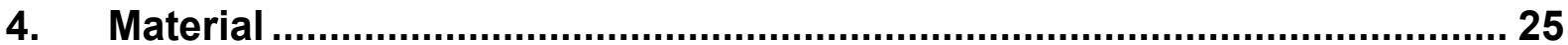

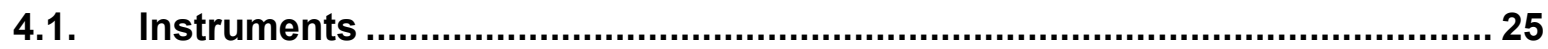

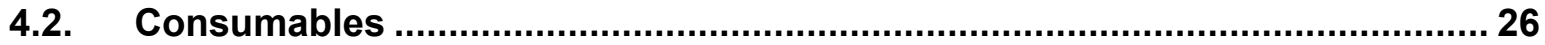

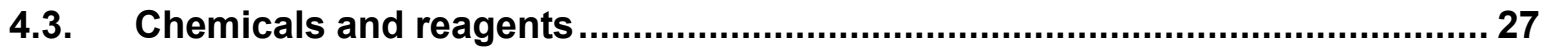

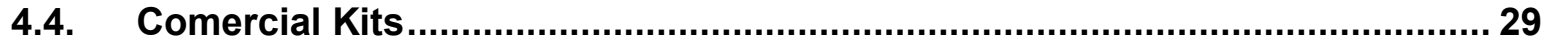

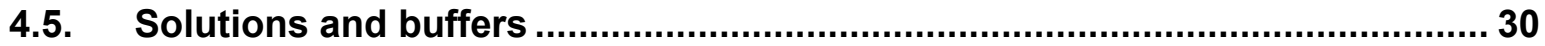

4.6. Molecular components (enzymes, cytokines, oligonucleotides, plasmids,

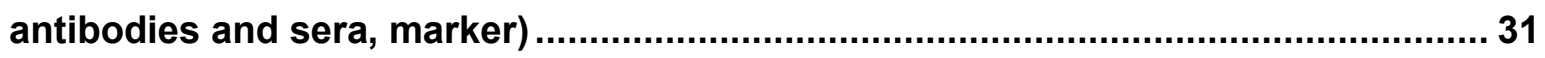

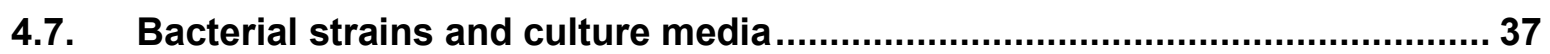

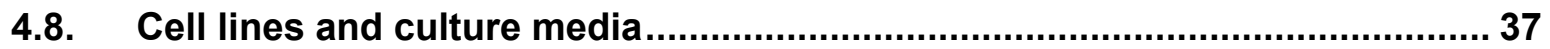

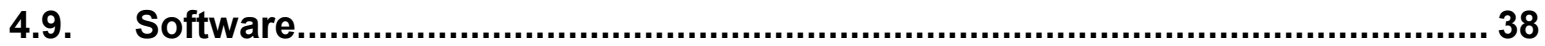

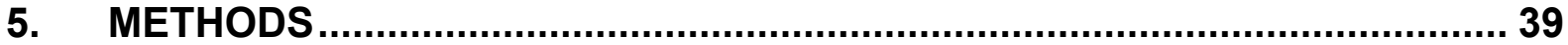

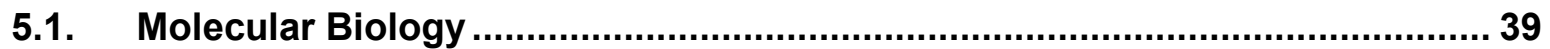

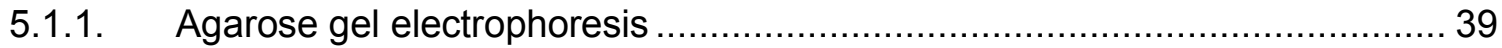

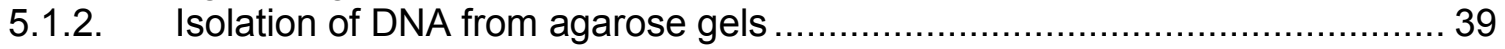

5.1.3. Restriction analysis of DNA fragments or plasmids ................................. 39

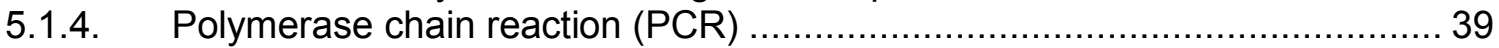

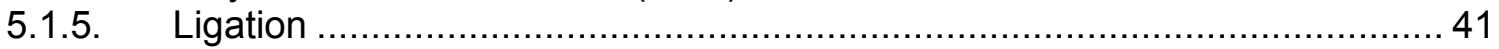

5.1.6. Transformation of bacteria ............................................................... 41

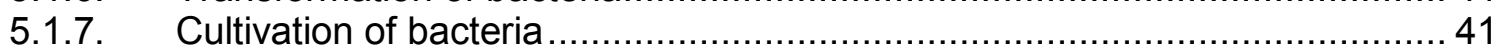

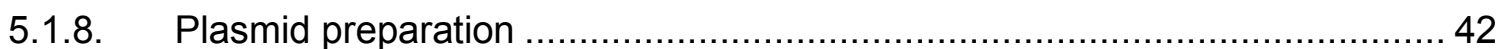

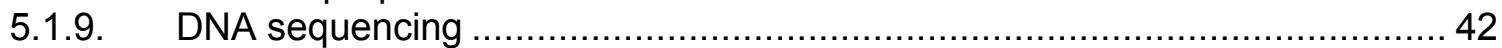

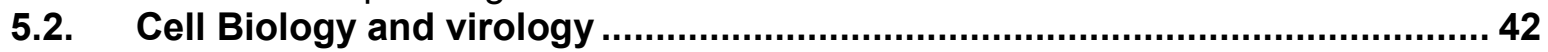

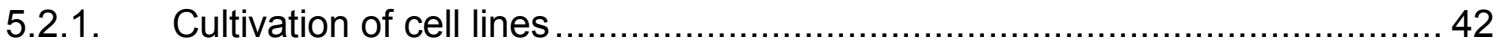

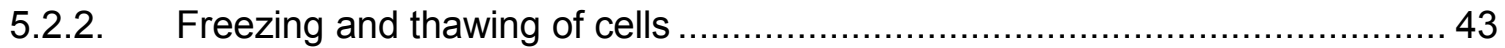

5.2.3. Transient transfection of cells ........................................................... 43

5.2.4. Isolation of human peripheral blood mononuclear cells (PBMCs) ................. 43

5.2.5. Isolation and activation of primary human B cells .................................... 44

5.2.6. Generation and concentration of lentiviral vectors ................................. 44

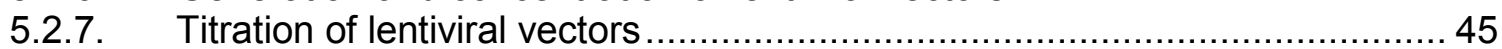

5.2.8. Transduction of adherent and suspension cells ................................... 46

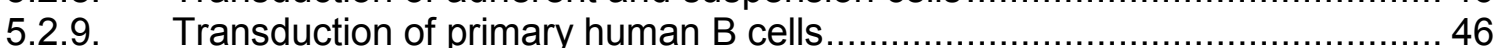

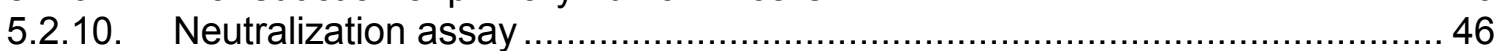


5.2.11. Cultivation and concentration of the Tupaia paramyxovirus ........................ 47

5.2.12. Fluorescent activated cell sorting (FACS) ........................................... 47

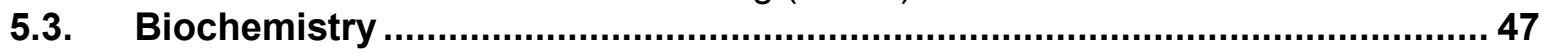

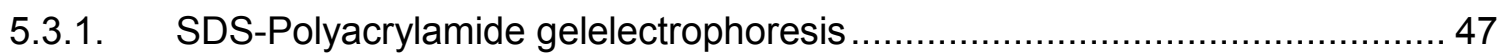

5.3.2. Western Blot analysis .................................................................... 48

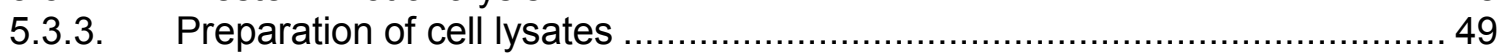

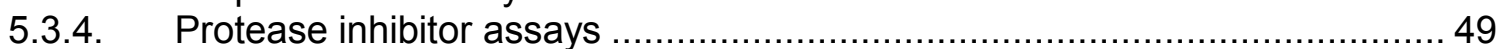

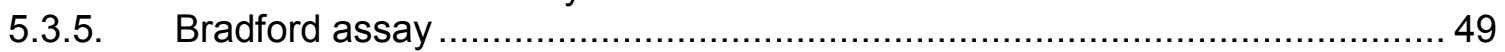

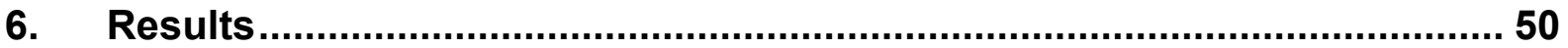

6.1. Generation and characterization of modified TPMV glycoproteins............... 50

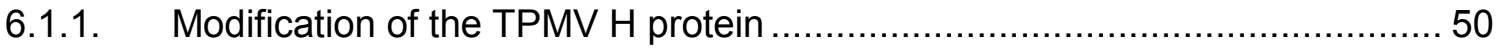

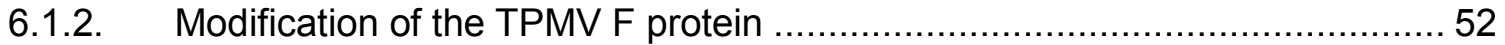

6.1.3. Surface expression of the modified TPMV H proteins................................ 53

6.1.4. Functional analysis of the truncated TPMV protein variants ........................55

6.2. Identification of a novel TPMV fusion protein fragment................................ 57

6.2.1. Further characterization of the TPMV $F$ protein with the novel antibody $F_{\text {ecto }} 57$

6.2.2. The novel F1a fragment is transported along the secretory pathway and

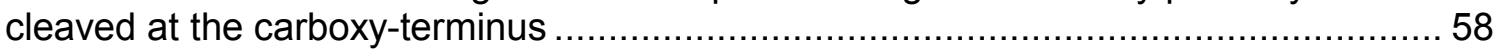

6.2.3. The TPMV F protein ectodomain is shed into the supernatant of cells..........61 61

6.2.4. The TPMV F1a fragment is present in TPMV virions ................................... 62

6.3. Characterization of the newly identified cleavage site ..................................63

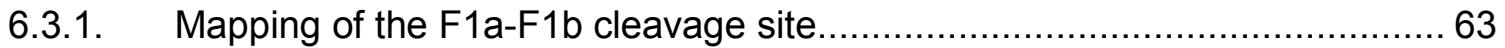

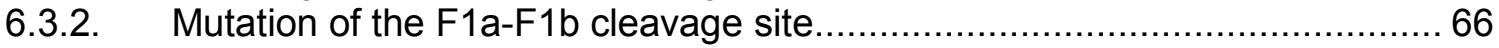

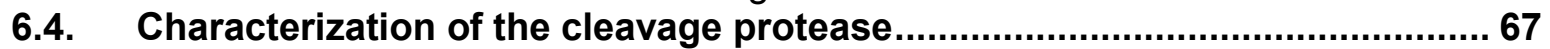

6.4.1. Screening of different protease inhibitors ................................................ 68

6.4.2. $\quad F$ protein cleavage is necessary for cell fusion ...................................... 71

6.5. Pseudotyping of lentiviral vectors with the modified TPMV proteins ............73

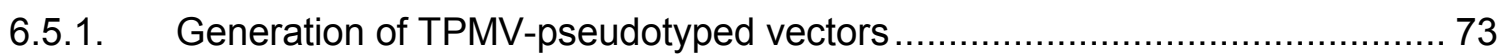

6.5.2. Screening of all TPMV $\mathrm{H}$ and $\mathrm{F}$ protein variants ....................................... 75

6.5.3. Determination of the optimal $\mathrm{H}$ to $\mathrm{F}$ ratio ................................................ 77

6.5.4. Confirmation of incorporation of the modified TPMV proteins into LVs ..........78

6.5.5. Targeting of different CD20-positive and negative cell lines ......................... 79

6.6. Stability assays of TPMV-pseudotyped lentiviral vectors .............................. 80

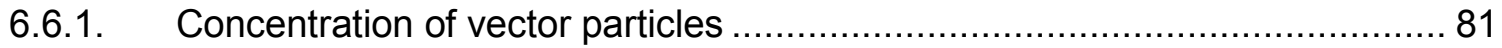

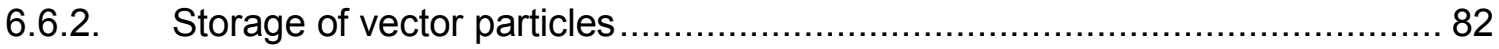

6.7. TPMV-pseudotyped vectors selectively transduce CD20-positive cells ........ 85

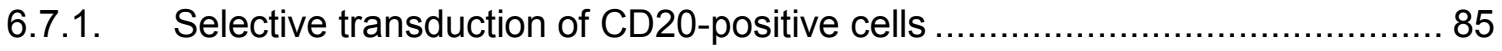

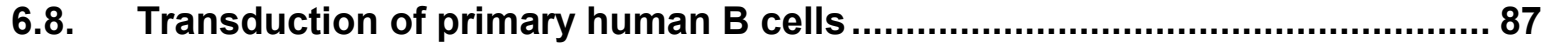

6.8.1. Transduction of activated primary human B cells................................ 87

6.8.2. Transduction of quiescent primary human $B$ cells................................. 89

6.8.3. Neutralization assays with retargeted LV-pseudotypes ............................ 91

7. Discussion......................................................................................... 93

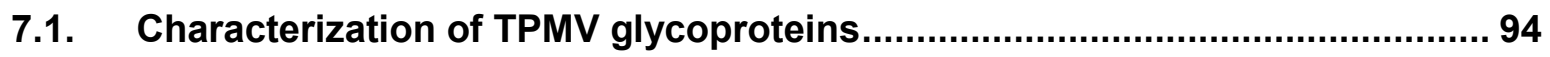

7.2. Pseudotyping of lentiviral vectors with TPMV glycoproteins ........................ 99

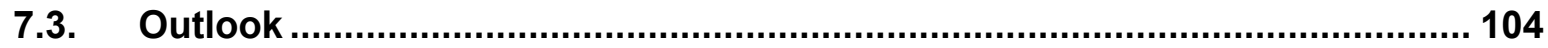

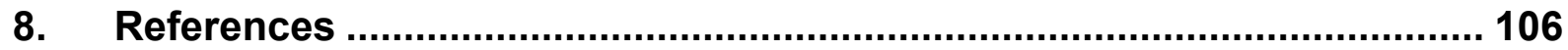




\section{A. SUMMARY}

Lentiviral vectors (LVs) are vectors of choice for many gene therapy applications since they mediate long term gene expression and can transduce dividing and nondividing cells. Recently, efficient targeting of LVs pseudotyped with the measles virus (MV) glycoproteins has been reported. However, MV antibodies in patients might limit the clinical use of these vectors. Thus, aim of this study was the development of targeted LVs pseudotyped with the glycoproteins of Tupaia paramyxovirus (TPMV). Since this animal paramyxovirus does not infect humans, no TPMV antibodies in patients are expected. For efficient incorporation in LVs, the TPMV glycoproteins, the hemagglutinin $(\mathrm{H})$ and fusion $(\mathrm{F})$ protein, were modified by truncation of their cytoplasmic tails. Targeting was achieved by displaying a single-chain antibody against the $\mathrm{B}$ cell surface marker $\mathrm{CD} 20$ on the $\mathrm{H}$ protein. The modified proteins were biochemically characterized and tested for their functionality. Unexpectedly, it was observed that an additional proteolytic cleavage of the $\mathrm{F}$ protein occurs during activation, resulting in the fragments $\mathrm{F} 1 \mathrm{a}, \mathrm{F} 1 \mathrm{~b}$ and $\mathrm{F} 2$. The newly identified fragment F1a was detected in virions and in supernatant of transfected cells. The F1a/F1b cleavage site was mapped and a cysteine protease was identified as likely activating protease. The data indicate that $F$ protein processing is more complex than expected.

After characterization, the modified TPMV glycoproteins were screened in all combinations for their ability to form functional pseudotyped LVs. Most efficient pseudotype formation was achieved with CT truncations of 80 amino acids (aa) for $\mathrm{H}$ $(\mathrm{H} \triangle 80 \mathrm{aCD} 20)$ and 32 aa for $\mathrm{F}(\mathrm{F} \triangle 32)$ (titers $\sim 10^{6}$ t.u. $\left./ \mathrm{ml}\right)$. The resulting vectors selectively transduced CD20-positive cells in a mixed cell population. Furthermore, they mediated efficient gene transfer into activated and quiescent primary human $B$ cells. Neutralization assays showed that TPMV-pseudotyped vectors were not neutralized by human sera containing MV antibodies.

In conclusion, it was demonstrated that targeted LVs pseudotyped with TPMV glycoproteins can be generated and escape neutralization by MV antibodies. Remarkably, the vectors are able to efficiently transduce even quiescent $B$ cells. Hence, they might be a valuable vector choice when systemic application of targeted lentiviral vectors in humans is required. 


\section{B. ZUSAMMENFASSUNG}

Lentivirale Vektoren (LV) sind für viele Anwendungen in der Gentherapie besonders gut geeignet, da das eingebrachte Gen über einen langen Zeitraum exprimiert wird und sie mitotisch aktive und inaktive Zellen transduzieren können. Kürzlich wurden zielgerichtete LV entwickelt, welche mit Masernvirus (MV)-Glykoproteinen pseudotypisiert sind. Allerdings würden MV-Antikörper in Patienten die klinische Anwendung dieser Vektoren wahrscheinlich erschweren. Deshalb wurden in dieser Arbeit LV entwickelt, welche mit den Glykoproteinen des Tupaia paramyxovirus (TPMV) pseudotypisiert sind. Da es sich dabei um ein für den Menschen nicht infektiöses Tier-Paramyxovirus handelt, werden keine Antikörper in Patienten gegen dieses Virus erwartet. Für einen effizienten Einbau der TPMV-Glykoproteine in LV, nämlich das Hämagglutintin $(H)$ und Fusionsprotein $(F)$, wurden die zytoplasmatischen Domänen (ZD) der Proteine verkürzt. Zielgerichteter Zelleintritt wurde ermöglicht, indem ein einkettiges Antikörper-Fragment (single chain antibody, scAb) gegen das B-Zell-Oberflächenmolekül CD2O an das H-Protein fusioniert wurde. Die modifizierten Proteine wurden biochemisch charakterisiert und auf ihre Funktionalität geprüft. Dabei wurde ein neues Fragment des F-Proteins detektiert (F1a), das aus einer unerwarteten zusätzlichen Spaltung des F-Proteins stammt und sowohl in Virionen als auch im Überstand transfizierter Zellen nachgewiesen wurde. Die entsprechende Spaltstelle wurde lokalisiert und eine Cystein-Protease als wahrscheinlich aktivierende Protease identifiziert. Die Daten deuten darauf hin, dass die Aktivierung des F-Proteins komplexer ist als ursprünglich gedacht.

Die modifizierten Glykoproteine wurden des Weiteren in allen Kombinationen darauf getestet, funktionale pseudotypisierte LV zu bilden. Am effizientesten war eine Verkürzung der $\mathrm{ZD}$ von 80 Aminosäuren (AS) für $\mathrm{H}(\mathrm{H} \triangle 80 \mathrm{aCD} 20)$ und $32 \mathrm{AS}$ für $\mathrm{F}$ (FA32) (Titer 106 t.u./ml). Die entsprechenden Vektoren transduzierten selektiv CD20-positive Zellen in einer gemischten Zellpopulation und außerdem aktivierte und ruhende primäre humane B-Zellen. Neutralisations-Experimente zeigten, dass die Vektoren nicht von Humanserum mit MV-Antikörpern neutralisiert werden.

Die in dieser Arbeit entwickelten Vektoren ermöglichen gezielten Zelleintritt und transduzieren bemerkenswerter Weise sogar ruhende B-Zellen. Folglich würden sie sich sehr für gentherapeutische Anwendungen in Menschen eignen. 


\section{ABBREVIATIONS}

$\alpha$

anti-

$a C D 20-s c A b \quad$ single-chain antibody directed against CD20

A

absorption or amper

$\mathrm{Aa}$

amino acids

Ac

acetate

Amp

ampicillin

APS

ammonium peroxydisulfate

ATCC

American Type Culture Collection

ATP

adenosine-5'-triphosphate

AZT

azidothymidine

BCR

B cell antigen receptor

BSA

Bovine Serum Albumin

${ }^{\circ} \mathrm{C}$

degree Celcius

ca.

circa

CMV

cytomegalovirus

CT

cytoplasmic tail

DMEM

Dulbecco's modified Eagle medium

DMSO

dimethyl sulfoxide

DNA

deoxyribonucleic acid

dNTP

Deoxyribonucleotide

DTT

dithio-1,4-threithol

ECACC European Collection of Cell Cultures

ECL

enhanced chemiluminescence

E. Coli

Escherichia Coli

EDTA

ethylene-diamine-tetra-acetate

e.g.

for example

EGF

epidermal growth factor

EGFR

epidermal growth factor receptor

ER

endoplasmatic reticulum

Env

envelope protein

et. al.

and others 


\begin{tabular}{|c|c|}
\hline $\mathrm{F}$ & fusion protein \\
\hline FACS & fluorescence activated cell sorting \\
\hline FCS & fetal calf serum \\
\hline FITC & fluorescence isothiocyanate \\
\hline FP & fusion peptide \\
\hline g & gram or gravitational acceleration \\
\hline gag & group specific antigen \\
\hline GALV & gibbon ape leukemia virus \\
\hline GFP & green fluorescent protein \\
\hline h & hour \\
\hline $\mathrm{H}$ & hemagglutinin \\
\hline HEK & Human embryonic kidney cells \\
\hline HEPES & 4-(2-hydroxyethyl)-1-piperazineethanesulfonic acid \\
\hline $\mathrm{HeV}$ & Hendravirus \\
\hline HIV-1 & human immunodeficiency virus-1 \\
\hline HRP & horseradish peroxidase \\
\hline ICLC & Interlab Cell Line Collection \\
\hline IL & Interleukin \\
\hline kDa & kilodalton \\
\hline I & liter \\
\hline LB & Luria-Bertani \\
\hline LV & lentiviral vector \\
\hline LTR & long terminal repeats \\
\hline $\mathrm{m}$ & milli- \\
\hline M & Molar \\
\hline $\mathrm{mAb}$ & monoclonal antibody \\
\hline MFI & mean fluorescence intensity \\
\hline$\mu$ & micro- \\
\hline $\min$ & minute \\
\hline MLV & murine leukemia virus \\
\hline MOI & multiplicity of infection \\
\hline MV & measles virus \\
\hline $\mathrm{n}$ & nano- \\
\hline
\end{tabular}


NEB New England Biolabs

NiV Nipahvirus

OD optical density

p.a. pro analysis

PBS phosphate buffered saline

PCR polymerase chain reaction

PE R-Phycoerythrin

PEI polyethylenimine

pol polymerase

$\Psi$

psi-packaging signal of retroviral genomic RNA

RNA ribonucleic acid

rpm rounds per minute

RPMI culture medium developed in the "Roswell Park Memorial Institute"

RT room temperature

scAb single-chain antibody

SDS sodium dodecyl sulfate

sec seconds

SFFV spleen focus forming virus

SIV simian immunodeficiency virus

SLAM signaling lymphocyte activation molecule

SP signal peptide

TBF Tupaia baby fibroblasts

TBS Tris-buffered saline

TEMED tetramethylethylenediamine

TM transmembrane domain

TPMV Tupaia paramyxovirus

Tris tris(hydroxymethyl)aminomethane

t.u. $\quad$ transducing units

U unit

UV ultraviolet

$\mathrm{V} \quad$ volt

VSV-G vesicular stomatitis virus-glycoprotein

WB Western Blot 


\section{INTRODUCTION}

\subsection{Lentiviral vectors for gene therapy}

Gene therapy is the treatment of a disease or a medical disorder by delivering genes into appropriate cells (Verma and Weitzman, 2005). Lentiviral vectors (LVs) are becoming a more and more attractive gene transfer system for the therapy of a variety of diseases, as they mediate long term gene expression and transduce both dividing and non-dividing cells (Matrai, Chuah, and VandenDriessche, 2010). These properties are advantageous in many gene therapeutic applications, because they allow stable transduction of, for example, terminally differentiated cells, like neuronal cells. LVs can accommodate large transgenes (up to $\sim 10 \mathrm{~kb}$ ) (Kaiser, 2003) and for example, change gene expression by introducing a therapeutic gene or kill a certain cell type by introducing suicide genes. Another strategy for therapy is to mediate antigen expression and presentation by transduction of dendritic cells with lentiviral vectors to activate the immune response of the host. Other possible target tissues include different areas of the brain, liver, muscle cells and bone marrow. While some cell types, mainly hematopoietic stem cells, can be transduced ex vivo and returned to the patient (Cartier et al., 2009), other cell types, like brain cells, can currently only be reached by direct injection of the viral vector.

Vectors based on retroviruses have already been tested in clinical trials. In the year 2000, eleven children with X-linked severe combined immunodeficiency (X-SCID) were cured by transduction of bone marrow with a vector based on the mouse leukemia virus (MLV), leading to correction of the defective gene (Cavazzana-Calvo et al., 2000). Unfortunately, some of the children developed leukemia due to insertional mutagenesis (Kaiser, 2003). Lentiviral vectors have been shown to harbour a lower risk of mutagenesis from transgene integration and therefore, they may substitute retroviral vectors in the future (Hematti et al., 2004; Modlich et al., 2009).

Currently, lentiviral vectors are applied in about $2.3 \%$ of clinical trials (http://www.wiley.com//legacy/wileychi/genmed/clinical/), and the number is increasing. Although these vectors are a promising tool, vector specificity and safety have to be improved. Targeted vectors that can be systemically administered to the patient and still only transduce selected cells would be a major improvement in vector technology (Waehler, Russell, and Curiel, 2007). 


\subsubsection{Vector design}

The first retroviral vectors developed for gene transfer were based on $y$-retroviruses such as the murine leukemia virus (MLV) (Mann, Mulligan, and Baltimore, 1983). These retroviral vectors were also used in the first approved human gene therapy trial to correct severe combined immunodeficiency (SCID) (Anderson, Blaese, and Culver, 1990; Blaese et al., 1993; Levine and Friedmann, 1991). In recent years, lentiviral vectors in contrast to $\mathrm{y}$-retrovirus-based vectors have become more and more popular. They are derived from lentiviruses that, like $\mathrm{Y}$-retroviruses, belong to the Retroviridae, a family of enveloped single-stranded (ss) RNA viruses of around 80 to $120 \mathrm{~nm}$ diameter (Vogt and Simon, 1999). The best known example of lentiviruses is the human immunodeficiency virus-1 (HIV-1). The genome consists of two copies of positive single- stranded RNA which, together with the viral replication enzymes, is enclosed by the capsid (Fig. 1). The viral envelope, that surrounds the capsid, is composed of the host cell membrane and complexes of the viral envelope protein Env which mediate attachment of the virus to its receptors on the host cell surface, enabling $\mathrm{pH}$-independent fusion of the viral and cellular membrane (Freed, 2007).

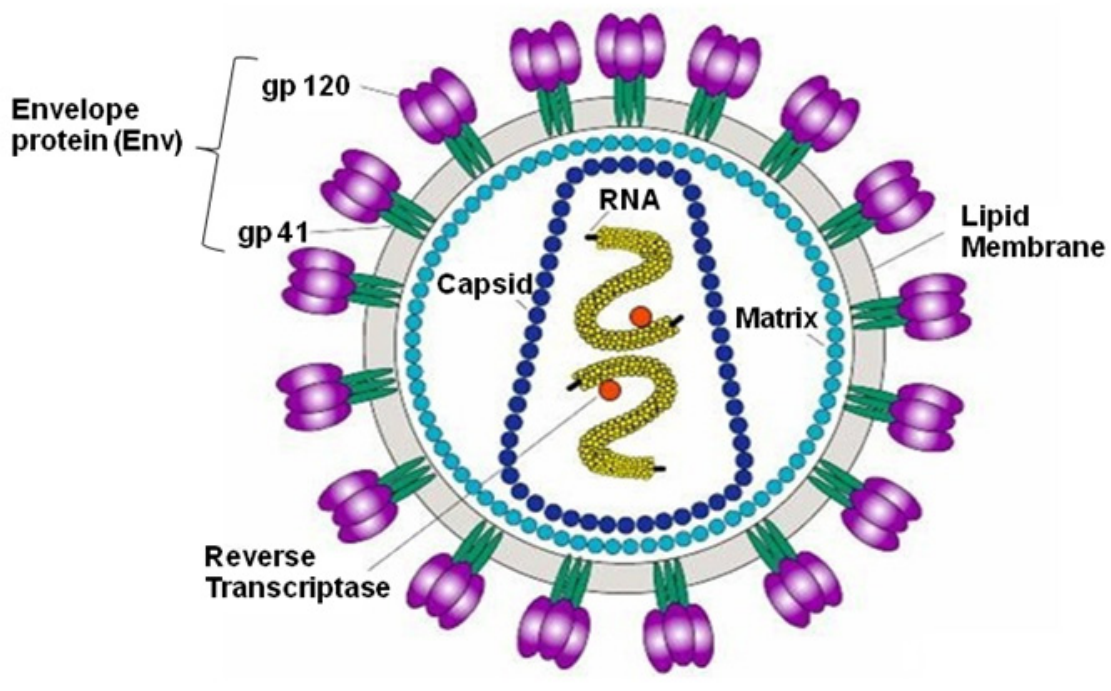

Figure 1: Schematic drawing of the lentivirus HIV-1

The two ssRNA molecules that are associated with the reverse transcriptase are indicated. They are surrounded by the capsid which is composed of the capsid protein p24. The membrane-associated matrix underlies the cell-derived lipid membrane with the inserted viral envelope proteins gp41 and gp120. (modified from http://www.charite.de/maximalmethodisch/e139/e84/hiv1.jpg 
The lentiviral genome is more complex than that of other retroviruses. It is organized mainly in the gag, pol and env genes but compared to simple retroviruses, lentiviruses have additional accessory genes that regulate viral gene expression, assembly and replication (Freed, 2007), namely tat, rev, nef, vif, vpu and vpr (Fig. 2). The Gag gene encodes the structural proteins including the membrane associated matrix protein, the core forming capsid protein and the viral RNA-binding nucleocapsid protein. The viral enzymes that accompany the ssRNA are encoded by the pol gene, including the reverse transcriptase, which transcribes the viral RNA to DNA, the integrase, that catalysis integration of the proviral DNA into the host genome and the protease which is responsible for gag-pol cleavage and virion maturation (Katz and Skalka, 1994). The env gene encodes the viral envelope.

\section{A Proviral structure of MLV genome $(8.8 \mathrm{~kb})$}

\begin{tabular}{|l|l|l|l|l|l|l|l|l|l|l|}
\hline $\mathrm{U} 3$ & $\mathrm{R}$ & $\mathrm{U} 5$ & $\Psi$ & gag & pro & pol & & $\mathrm{U} 3$ & $\mathrm{R}$ & $\mathrm{U} 5$ \\
\hline
\end{tabular}

\section{B Proviral structure of HIV-1 genome $(9.7 \mathrm{~kb})$}

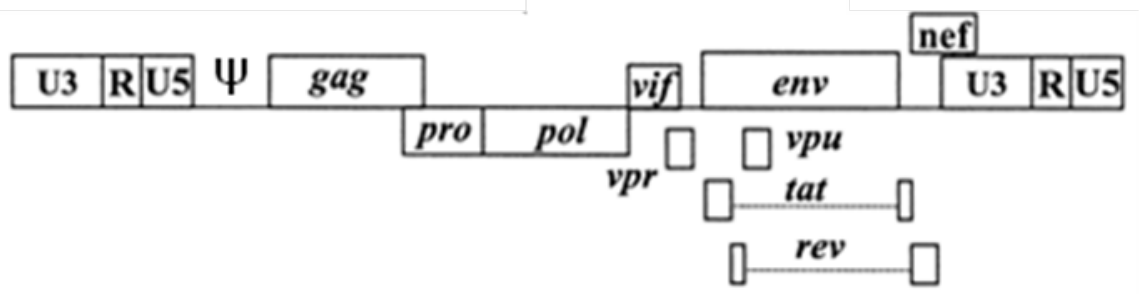

Figure 2: Schematic representation of the MLV and HIV-1 genome

A: Proviral structure of MLV genome; B: Proviral structure of HIV-1 genome LTR: long terminal repeats; $\psi$ : encapsidation signal psi (modified from (Hu and Pathak, 2000))

On both ends of the retroviral genome there are redundant sequences called long terminal repeats (LTRs) (Coffin, 1996; Vogt, 1997) that can be further divided into U3 (unique 3'), R (repeat) and U5 (unique 5') regions (Fig. 2). The viral promoter, transcription enhancers, a transcription termination signal and polyadenylation signals are located in these regions. The LTRs also contain short sequences, the attachment sites that are important for integration of the viral DNA into the host 
chromosomes (Coffin, 1996). The packaging signal $\psi$ or encapsidation signal contains sequences that interact with viral proteins to mediate packaging of the genomic RNA into newly formed virions (Watanabe and Temin, 1982).

The life cycle of lentiviruses can be separated into different steps (Fig. 3): First, the virus binds to its receptors via the glycoproteins of the viral envelope and fuses with the cell membrane. Afterwards, the virus is uncoated and the viral core containing genomic RNA is released into the cytoplasm where the viral ssRNA is reverse transcribed into a dsDNA copy. The dsDNA within the core is then actively transported to the nucleus (Bukrinsky et al., 1993) and integrated into the host genome as a provirus. This active transport is one of the major advantages of lentiviruses over retroviruses, because this is the reason why they are able to infect not only dividing but also non-dividing cells. In contrast, other retroviruses are dependent on cell division and dissolution of the nucleus to integrate their reverse transcribed dsDNA copies. After integration of proviral DNA, the cellular transcription and translation machinery is used to express the viral genes and to generate RNA encoding all viral proteins. This newly generated viral RNA is packaged and a new virus is assembled which leaves the host cell by budding.

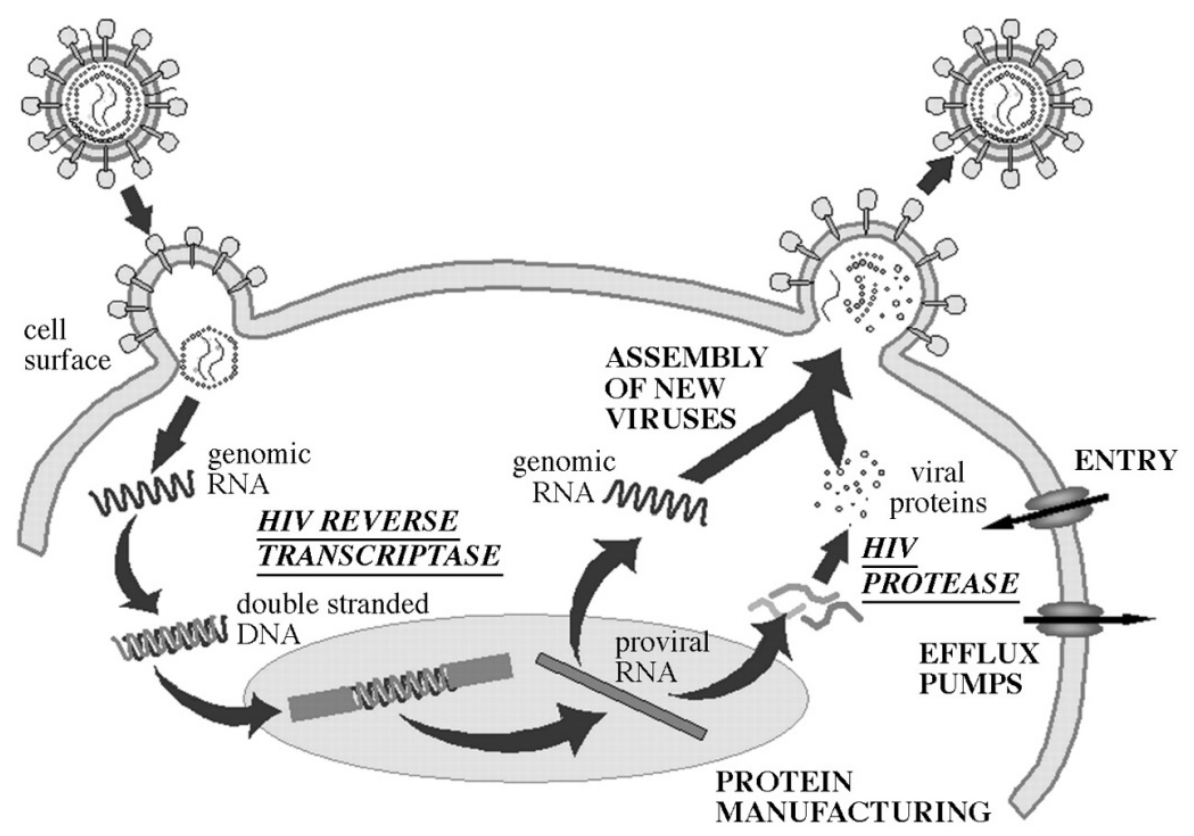

Figure 3: Life cycle of HIV-1

The virus binds to the cell surface and fuses with the host membrane. After entering the host cell, reverse transcriptase produces proviral double-stranded DNA that is transported to the nucleus. The DNA is integrated and viral RNA is synthesized for the production of viral proteins. At the cell membrane, assembly occurs and the virus leaves the cell by budding. (Hoggard and Owen, 2003) 
Due to the lentiviral life cycle, it is obvious why they are becoming more and more popular as vectors in gene therapy. They are very efficient in gene transfer, and integration of the transferred genes into the host genome allows long-term gene expression. Vectors derived from lentiviruses are replication-deficient and can carry any gene of interest into a target cell, a process called transduction.

When generating lentiviral vectors some important safety issues have to be considered. First, all non-essential genes, like genes for the accessory proteins and for virulence should be removed from the vector sequence. Second, sequences necessary for vector RNA synthesis, packaging, reverse transcription and integration of cDNA should be split onto a different plasmid than elements encoding the viral enzymes and structural proteins. These so called cis- and trans-acting sequences should have a minimum of sequence overlap to reduce the risk of homologous recombination and thereby generation of replication-competent vectors. In trans, the genes for the polyproteins gag and pol have to be provided. The resulting plasmid is called packaging vector (Fig. 4, A). In cis-acting sequences, including the 5' and 3' LTR, the packaging signal $\psi$ and the gene of interest which is under the control of a chosen promoter, are present on a plasmid called transfer vector (Fig. 4, B). The genes encoding the envelope proteins are provided on a third plasmid, the envelope vector (Fig. 4, C). The packaging vector and the envelope vector plasmids both lack a functional $\psi$-site and are therefore not packaged into vector particles.

The packaging vector plasmids are divided into "generations" depending on the viral sequences provided (Fig. 4, A). The first generation plasmid contains the gag and pol sequences, the viral regulatory genes tat and rev and all accessory genes (vif, vpr, $v p u$ and nef). To improve lentiviral vector safety, in the second generation the four accessory genes were removed, leaving only the regulatory genes tat and rev (Zufferey et al., 1997). Tat protein increases the level of viral RNA during production by activating the LTRs and Rev protein interacts with viral RNA containing a Rev Responsive Element (RRE) to be transported from the cell nucleus to the cytoplasm. In the third generation, the rev gene is split from the gag and pol sequences and expressed from a separate plasmid (Dull et al., 1998).

The transfer vector plasmids can be modified by deleting promoter/enhancer sequences in the U3 region of the 3' LTR, resulting in a self-inactivating (SIN) vector (Miyoshi et al., 1997; Zufferey et al., 1997) (Fig. 4, B). During reverse transcription, this deletion is reproduced in the 5' LTR and therefore transcription of the provirus is 
inactivated and no packagable RNAs can be produced (Fig. 4, D and E). This modification decreases the risk of replication competent lentiviruses. Additionally, LTR-mediated insertional activation of proto-oncogenes is reduced due to the deleting of the enhancer elements. One of the disadvantages of this vector system is the low transcription activity of the internal promoter of the transgene, in contrast to the promoter/enhancer sequences of the LTR. To overcome this problem and to further improve vector safety, the choice of the promoter is very important, for example, to set the transfer gene under control of an inducible or a cell-specific promoter (transcriptional targeting).

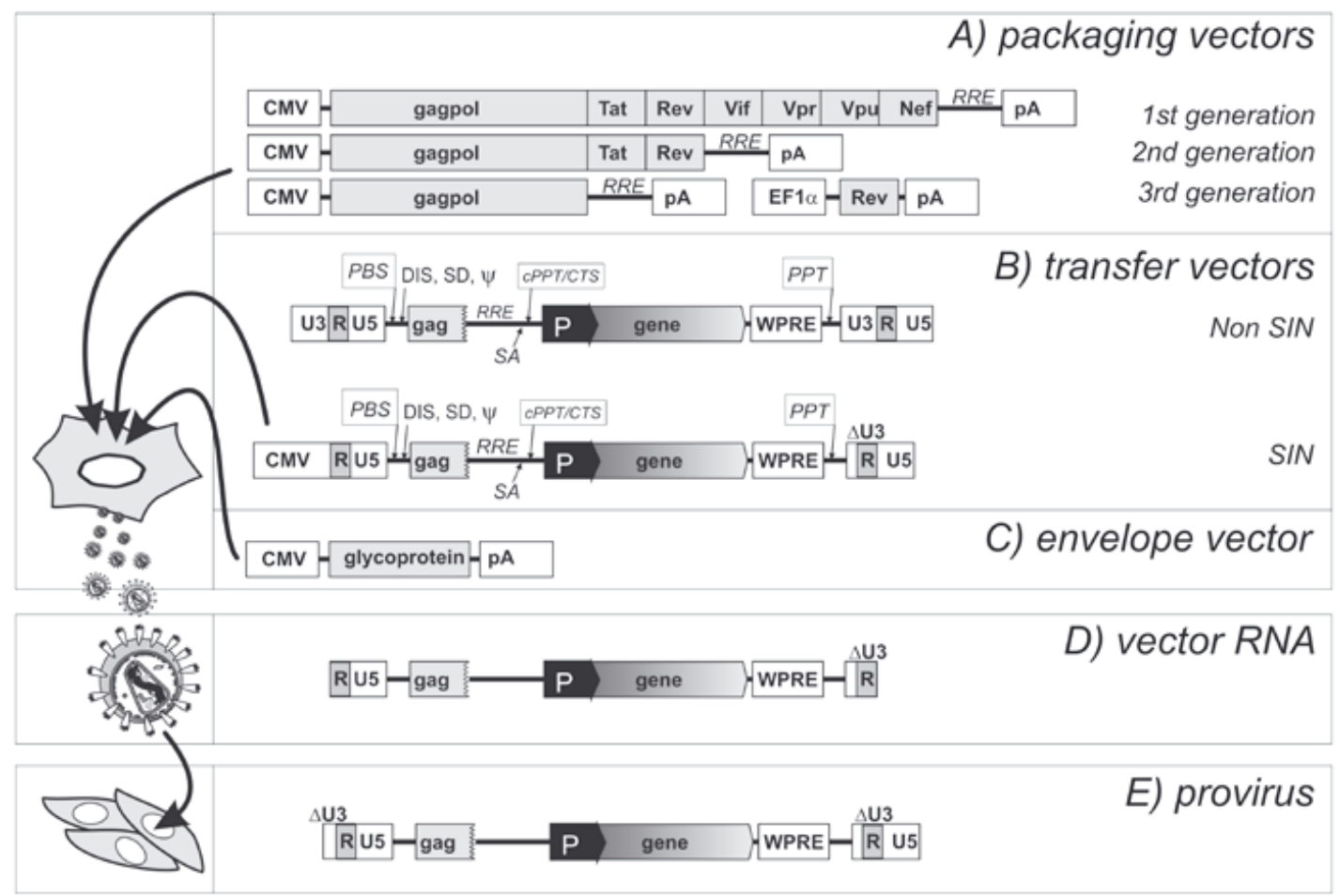

Figure 4: Gene delivery system based on lentiviral vectors

A-C: Different plasmids for production of lentiviral vectors and genes they contain are shown.

D: Schematic drawing of the RNA that the produced vector contains. E: Sequences integrated by the vector in host genome (provirus). CMV: cytomegalovirus immediate-early promoter; EF1a: human elongation factor 1- $\alpha$ promoter; gag: 5 ' portion of gag gene containing dimerization/packaging signals; PBS: primer binding site; DIS: dimerization signal; SD: splice donor site; SA: splice acceptor site; $\psi$ : packaging signal; CPPT: central polypurine tract; CTS: central termination sequence; RRE: Rev response element; PPT: polypurine tract; pA: polyadenylation signal; $\triangle \mathrm{U} 3$ : SIN deletion in U3 region of 3' LTR; P: internal promoter for transgene expression; WPRE: woodchuck hepatitis virus (WHV) posttranscriptional regulatory element. (Pluta and Kacprzak, 2009)

For the production of lentiviral vectors, the packaging plasmid, the transfer plasmid carrying the transgene, e.g. an eGFP-encoding gene and a plasmid encoding the 
envelope are transiently co-transfected in packaging cells, most commonly these are human embryonic kidney (HEK) 293T cells (Fig. 5) (Naldini et al., 1996).

Vector particles are released into the supernatant of the producer cells that have incorporated the RNA of the transfer vector but do not contain the genetic material for the core and envelope proteins. Since the viral vectors bud from the cell membrane, the viral envelope is composed of the cellular lipid-bilayer and the incorporated viral envelope proteins. The vector-containing cell supernatant can be used either immediately or concentrated for transduction.

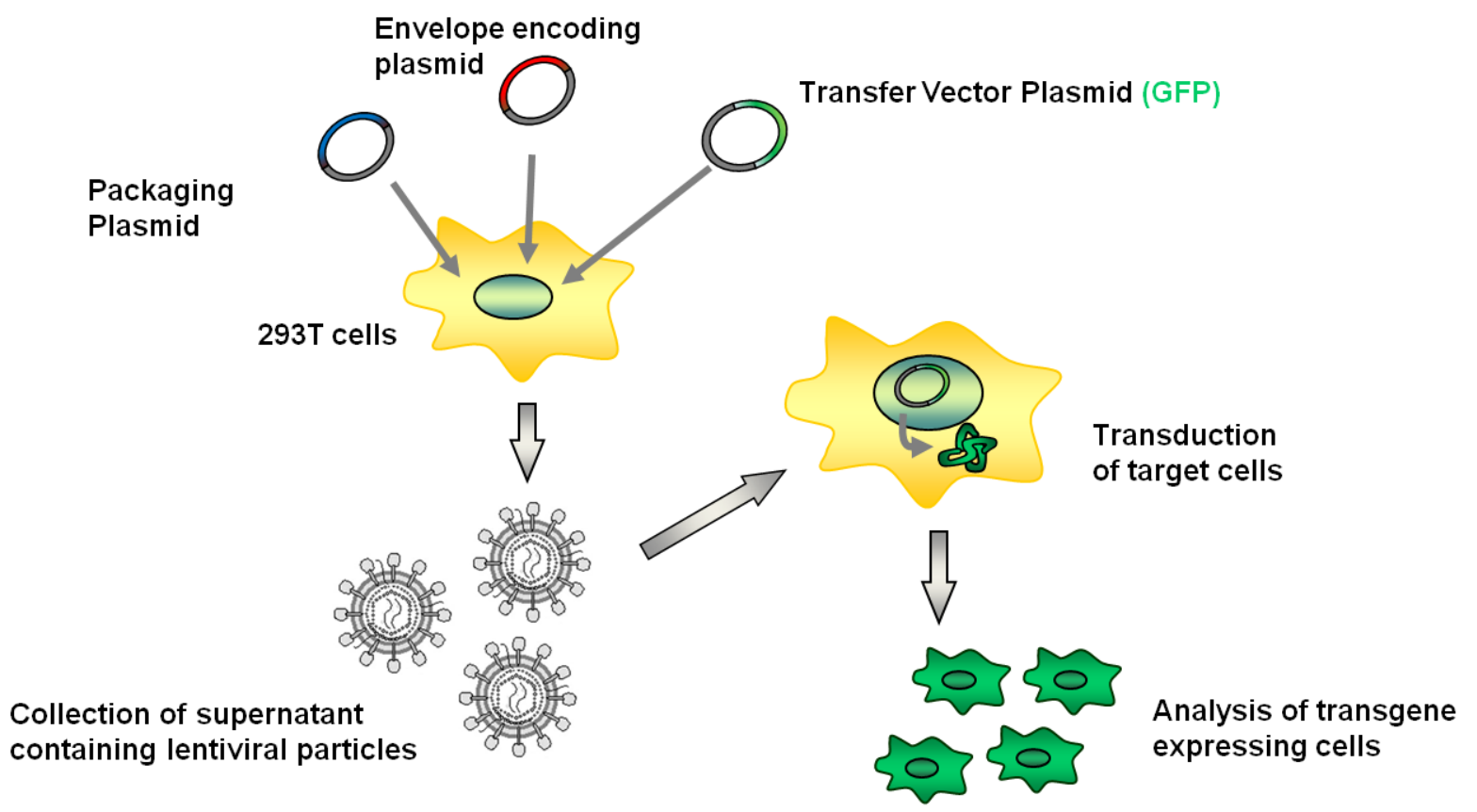

Figure 5: Production of lentiviral vectors

Packaging cells (here 293Tcells) are transfected with a packaging plasmid, a transfer vector plasmids and a plasmid encoding an envelope protein. After 24-48 hours, the viral particle containing supernatant of the producer cells is harvested and used for transduction of target cells. Transduced cells are finally analyzed for transgene expression, e.g. GFP. GFP: green fluorescence protein

Tropism of lentiviral vectors is determined by the viral envelope protein. It interacts with its receptor on the target cell membrane and triggers fusion. Since the HIV-1 wildtype envelope protein has only restricted tropism, glycoproteins derived from other viruses are often used for lentiviral production. 


\subsubsection{Pseudotyping of lentiviral vectors}

One of the great advantages of lentiviral vectors is that their natural tropism can be altered by exchanging the native envelope protein with glycoproteins from different viruses, a process called pseudotyping (Fig. 6).
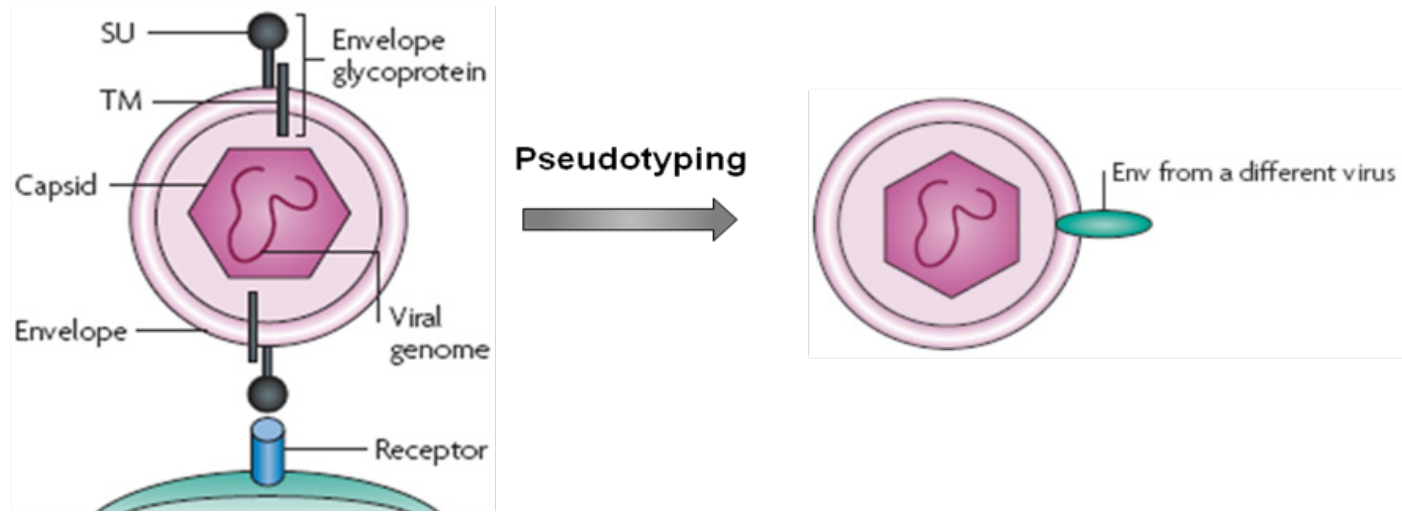

Figure 6: Pseudotyping of lentiviral vectors

A lentivirus binds to a receptor via specific glycoproteins. This interaction determines the host range of the virus. Pseudotyping means changing the tropism by replacing the viral attachment glycoprotein with that of a different virus. SU: surface subunit; TM: transmembrane subunit; Env: envelope glycoprotein (modified from (Waehler, Russell, and Curiel, 2007))

Lentiviral vectors are highly permissive for incorporation of glycoproteins derived from other viruses (Cronin, Zhang, and Reiser, 2005; Schnierle et al., 1997). The pseudotyped particles possess the ability to transduce target cells of the virus from which the envelope protein was derived. Consequently, pseudotyping allows infection of target cells that the virus does not naturally infect.

The key event for pseudotyping lentiviral vectors was the observation made when HIV-1 was produced in cells infected with different viruses, e.g. amphotropic murine leukemia virus (MLV) (Chesebro, Wehrly, and Maury, 1990; Spector et al., 1990) or herpes simplex virus (HSV) (Zhu, Chen, and Huang, 1990). The resulting virions showed an expanding host range, suggesting that envelope proteins of MLV and HSV were incorporated.

The molecular events occurring during pseudotyping of lentiviral vectors are still not fully understood. There are two mechanisms proposed that lead to incorporation of viral proteins in viral vectors, an active and a passive mechanism. For active incorporation, direct interactions of the cytoplasmic tail (CT) of the envelope protein 
and components of the virion core or indirect interactions via cellular factors with viral gag proteins are required (Murakami and Freed, 2000a; Murakami and Freed, 2000b; Swanstrom and Wills, 1997). During passive incorporation, a nonspecific interaction between the cytoplasmic tail and the viral core takes place, assuming that there is no steric incompatibility of the CT with viral assembly and that there are sufficient amounts of envelope proteins presented at the site of virus budding (Swanstrom and Wills, 1997). Lipid rafts play an important role in pseudotyping. It was shown that many viruses such as C-type retrovirus, Lentivirus and Paramyxoviurs reassemble their envelope in these microdomains of the plasma membrane (Pickl, PimentelMuinos, and Seed, 2001). As a consequence, the envelope proteins of other viruses and cellular components are incorporated into viral particles during co-infection.

Today, the most common envelope protein used for pseudotyping is the glycoprotein of vesicular stomatitis virus, VSV-G. The possibility of pseudotyping LVs with VSV-G was first demonstrated 1996 independently by three different groups (Akkina et al., 1996; Naldini et al., 1996; Reiser et al., 1996). VSV-G-pseudotyped vectors have a high stability, a broad host range and high titers can be generated. However, since the aim of viral gene therapy is to target only specific cells or tissues, the broad tropism of VSV-G can be disadvantageous. For specific targeting of cells, glycoproteins of viruses that naturally infect only one cell type are often used. Examples are transduction of neuronal tissue by lyssavirus-pseudotyped LVs (Desmaris et al., 2001; Watson et al., 2002) or airway epithelium transduction by filovirus-pseudotypes (Chan et al., 2000; Kobinger et al., 2001).

One of the problems of pseudotyping LVs is that often the titers reached by pseudotyped lentiviral vectors are very low due to insufficient incorporation of heterologous glycoproteins. To overcome this problem the cytoplasmic tails of some glycoproteins have to be modified. As mentioned before, CT interactions with the viral core may play an important role in glycoprotein incorporation. It was shown that most mammalian and avian retroviruses such as HIV, MLV and ALV (avian leukemia virus) require proteolytic cleavage of the R-peptide, a short amino acid sequence (16 aa) at the C-terminus of CT of the envelope glycoprotein for activation (Bobkova et al., 2002; Green et al., 1981; Henderson et al., 1984; Schneider et al., 2011). Pseudotyping of retroviral vectors using paramyxovirus glycoproteins requires truncation of the proteins' cytoplasmic tails. The fusion $(F)$ and attachment $(H)$ proteins of the measles virus can only be incorporated into lentiviral vectors when 
their cytoplasmic tails are truncated (Funke et al., 2008a; Funke et al., 2009). In another example the $\mathrm{F}$ (fusion) and $\mathrm{HN}$ (hemmagglutinin-neuraminidase) protein of Sendai virus were efficiently incorporated into a simian immunodeficiency virus (SIV) derived vector after truncation of the $F$ protein cytoplasmic tail. Additionally, in this system the CT of the SIV envelope protein was added to the CT of the hemmagglutinin-neuraminidase (Kobayashi et al., 2003). Recently, another study was published where a chimeric glycoprotein was used to pseudotype a lentiviral vector. In this study, the cytoplasmic tail of rabies virus glycoprotein (RVG) was replaced with the cytoplasmic region of VSV-G. The resulting vectors showed an increase in transduction efficiency due to higher levels of glycoprotein incorporation and efficiently transduced the central nervous system of rats in vivo (Carpentier et al., 2011).

To date, glycoproteins of many virus families have been used for pseudotyping. But often, the specificities of the used glycoproteins are not sufficient to transduce only one certain cell type. To further narrow the tropism of viral vectors for gene therapy, additional modifications are necessary.

\subsubsection{Targeting strategies for lentiviral vectors}

Lentiviral vectors pseudotyped with heterologous glycoproteins allow transduction of a certain cell type or tissue, dependent on the tropism of the virus the glycoproteins are derived from. Since many viruses naturally infect more than one kind of tissue or cell type, different techniques to further increase efficiency and specificity of vectors were developed. One of the targeting strategies to restrict tropism is the use of specific promoters that are active only in target cells, a process called transcriptional targeting (Waehler, Russell, and Curiel, 2007). Examples for the use of LVs with cell type specific promoters are expression of a transgene in endothelial cells (De Palma, Venneri, and Naldini, 2003), in retinal cells (Miyoshi et al., 1997) or liver tissue (Oertel et al., 2003; VandenDriessche et al., 2002).

Another strategy is the development of protease-activatable viral vectors. For targeting, a polypeptide is fused to the envelope proteins that blocks attachment or fusion of the vector. Specific proteases on the cell surface are required that cleave the inhibitory peptide, resulting in gene transfer into the target cell (Szecsi et al., 2006). 
One of the most desirable strategies remains transductional targeting, which means targeting on the entry level of the viral vector. This technique leaves other tissues or cells completely unaffected and can be achieved for example by the insertion of celltype specific ligands or other molecules into the viral envelope, like growth factors (EGF), single chain antibodies (scAbs), cytokines or even receptors. The most common approach to alter the tropism of a lentiviral vector is the use of single chain antibodies. These molecules consist of the variable regions of one heavy and one light chain of an immunoglobulin that are connected with a serine-glycine linker and have the same specificity as the original molecule they were derived from. Lentiviral vectors pseudotyped with Sindbis virus glycoproteins are an example for targeting using antibodies. The envelope of Sindbis virus consists of the E1 and E2 protein, which are responsible for attachment and fusion. Targeting was achieved by a monoclonal antibody that was non-covalently bound to the glycoproteins of the viral envelope (Morizono et al., 2005). Since Sindbis virus uses more than one receptor for cell entry, in this approach the receptor binding sites of the E2 protein were mutated, resulting in an engineered virus, that is unable to recognize its natural receptor. One disadvantage of this system is the $\mathrm{pH}$-dependent membrane fusion and the vector instability due to the non-covalent bond between the antibody and the glycoproteins. Sindbis virus enters the cell by receptor-mediated endocytosis which is required for E1-E2 activation. Subsequent fusion is induced by low $\mathrm{pH}$ in the endosomes.

Recently, efficient targeting of lentiviral vectors pseudotyped with glycoproteins of a paramyxovirus, the measles virus (MV) $\mathrm{F}$ (fusion) and $\mathrm{H}$ (attachment) proteins has been reported (Anliker et al., 2010; Frecha et al., 2008; Funke et al., 2008a; Funke et al., 2009; Munch et al., 2011). In contrast to Sindbis virus, the envelope proteins of MV mediate $\mathrm{pH}$-independent membrane fusion and endocytosis for glycoprotein activation is not required. Targeting of $\mathrm{MV}$ is based on the covalently bond of specificity domains on the viral hemagglutinin (Cattaneo et al., 2008; Navaratnarajah, Leonard, and Cattaneo, 2009). However, to achieve full targeting, modifications of the $\mathrm{H}$ protein to avoid binding to its natural receptors CD46, SLAM and the recently identified nectin 4 are necessary (Nakamura et al., 2005; Noyce et al., 2011; Vongpunsawad et al., 2004). A potential disadvantage of the MV system is the presence of neutralizing antibodies in nearly all patients, either by previous infection with wild-type virus or by vaccination. In an animal colon carcinoma model, the 
therapeutic effect of oncolytic MV vectors was abrogated in animals vaccinated against MV; however when the MV glycoproteins were replaced by those of the related animal paramyxovirus Canine distemper virus (CDV), the oncolytic effect was restored (Miest et al., 2011).

Nevertheless, glycoproteins of measles virus have great potential to pseudotype lentiviral vectors, since their fusion and attachment functions are separated into two proteins and retargeting can be achieved by displaying small molecules on the $\mathrm{H}$ protein. It has to be investigated, if other paramyxovirus envelope proteins, for example of non-human viruses that will not be neutralized by preexisting antibodies in patients, can also be targeted and used to pseudotype lentiviral vectors.

\subsection{Paramyxoviruses}

The Paramyxoviridae are a family within the order Mononegavirales. They are enveloped negative-strand RNA viruses that are widespread among humans and animals. The family of Paramyxoviridae is further classified into the two subfamilies Paramyxovirinae and Pneumovirinae. Genera of Paramyxovirinae are Respirovirus, Rubulavirus, Avulavirus, Morbilivirus and Henipavirus. Pneumovirinae contains two genera, Pneumovirus and Metapneumovirus. Each of the five genera of Paramyxovirinae includes species that are highly pathogenic for humans. Most prominent examples are the measles virus, which is a Morbilivirus, mumps virus (Rubulavirus) and Sendai virus (Respirovirus). In recent years, the Hendra and Nipah viruses (Henipaviruses) were identified which cause deadly diseases in animals and humans. The most famous member of Pneumovirinae is the Human respiratory syncytial virus (HRSV) which is often the causative agent for respiratory infections in young children (Counihan et al., 2001). There are several species of the paramyxoviruses that have not yet been classified into genera, for example $J$ virus, Mossmann virus and the Tupaia paramyxovirus (Lamb, 2007).

All members of this virus family have a similar viral structure (Fig. 7). They are enveloped viruses with a diameter of 150 to $300 \mathrm{~nm}$ and in some cases even greater than $1 \mu \mathrm{m}$ (Goldsmith et al., 2003). The envelope is a cell-derived lipid bilayer in which two kinds of virus-derived glycoproteins are embedded, the attachment protein and the fusion protein. The attachment glycoproteins are the HN (hemagglutininneuramidase) for Respiroviruses, Rubulaviruses and Avulaviruses, the $\mathrm{H}$ 
(hemagglutinin) for Morbilliviruses and the $G$ (glycoprotein) for Henipaviruses and members of Pneumovirinae. Dependent on the genera, the attachment proteins possess hemagglutination activity only $(\mathrm{H})$, or hemagglutination and neuraminidase activity (HN) or none of these activities (G). The other glycoprotein is the $F$ (fusion) protein that mediates fusion of the viral envelope with the cell membrane. Fusion occurs $\mathrm{pH}$-independently. The glycoproteins are abundantly incorporated into the envelope and form spike-like complexes. The genome is enclosed by the envelope and consists of a non-segmented, negative RNA strand which is bound to the nucleocapsid to form helical structures called ribonucleoproteins (RNPs). The matrix (M) protein which organizes virus assembly and maintains viral structure is assembled between the envelope and the nucleocapsid.

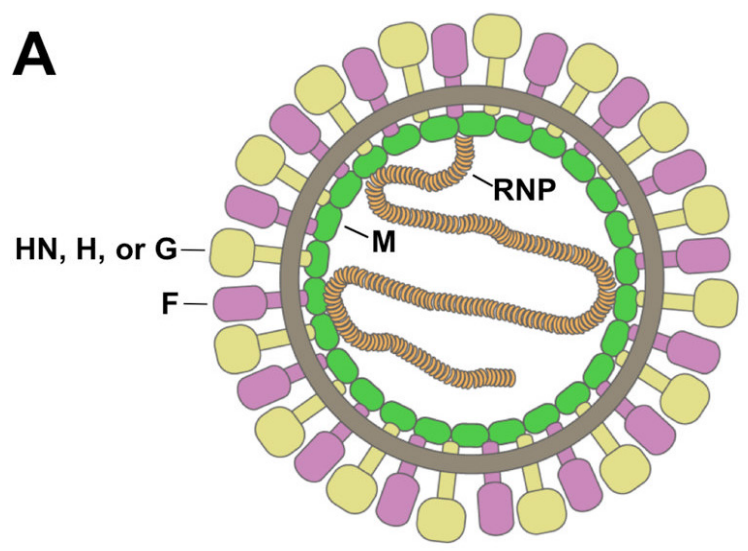

\section{Figure 7: Schematic representation of a paramyxovirus}

The $M$ protein is underlying the inner surface of the lipid envelope in which the viral glycoproteins $(H N$, $\mathrm{H}$ and $\mathrm{F}$ or $\mathrm{G}$ ) are embedded. The RNP is associated with the $\mathrm{M}$ protein and contains the negativestrand single-stranded RNA genome, the viral RNA-dependent RNA-polymerase and the $\mathrm{N}$ and $\mathrm{P}$ proteins. HN: hemagglutinin-neuraminidase; $\mathrm{H}$ : hemagglutinin; G: glycoprotein; F: fusion protein; $\mathrm{M}$ : matrix protein; RNP: ribonucleoproteins (Harrison, Sakaguchi, and Schmitt, 2010)

The life cycle of paramyxoviruses (Fig. 8) starts with binding of the virus to a receptor molecule on the surface of a target cell, followed by fusion of the viral membrane with the target cell membrane. Afterwards, the virion content including the RNP is released into the cytoplasma of the target cell and viral transcription starts. The viral RNA-dependent RNA polymerase complexes produce individual messenger RNAs from the negative-sense viral genomic RNA that are then translated into viral proteins. 
Genes closest to the 3' end are transcribed more abundantly than those towards the 5' end, because the RNA polymerase often dissociates from the RNA genome and has to reenter it at the $3^{\prime}$ end. As a result, there is a gradient of gene expression regulated by the position of each gene relative to the single promotor (Cattaneo et al., 1987a; Whelan, Barr, and Wertz, 2004). The negative-sense viral genomic RNA not only serves as a template for transcription but also for replication. First, positivesense antigenomes are produced that are followed by production of negative-sense genomes from the antigenome template. The newly synthesized genomic RNA together with the newly produced viral proteins is transported to the cell membrane where assembly occurs and new viruses leave the infected cell by budding.

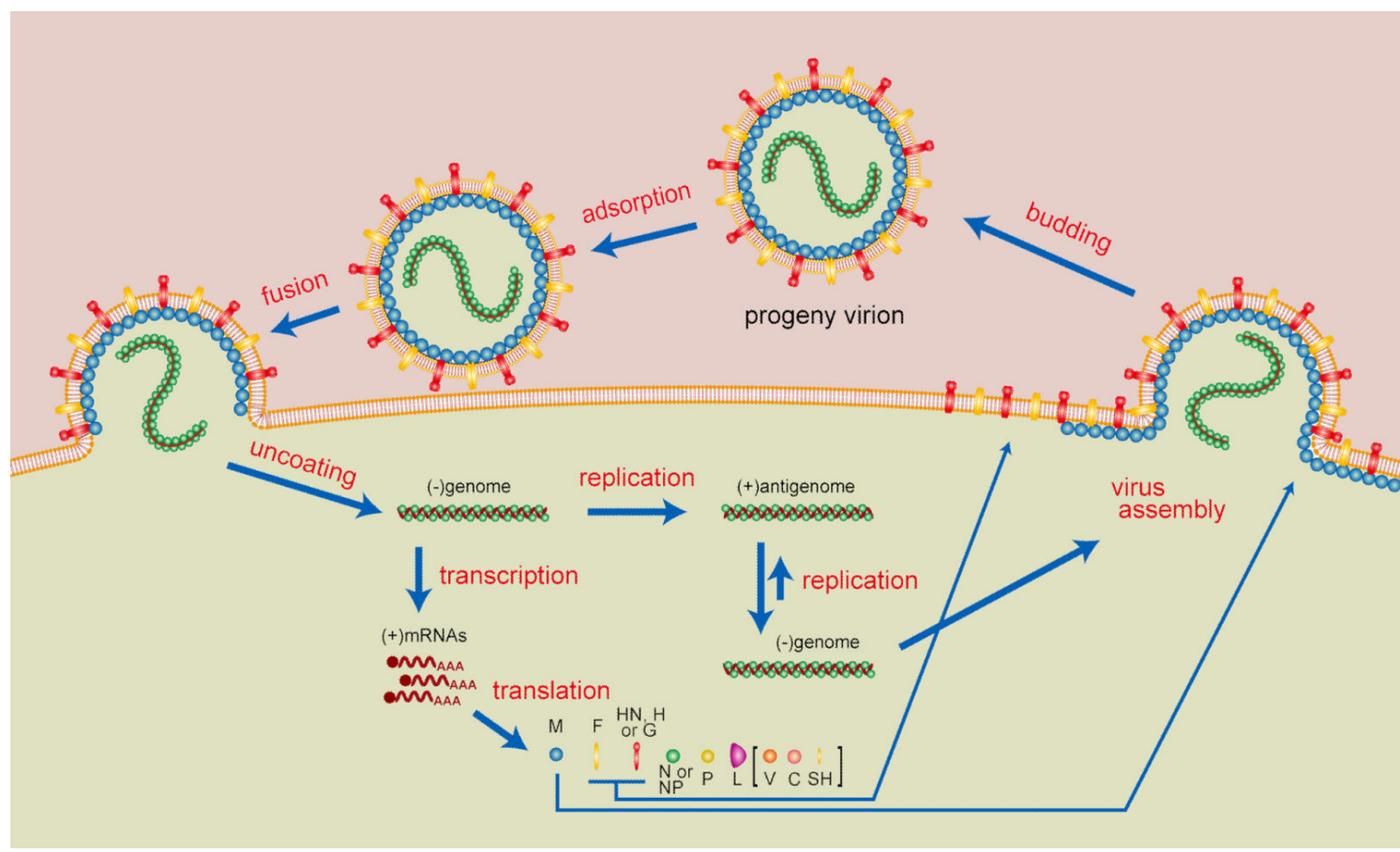

Figure 8: Life cycle of paramyxoviruses

After attachment and fusion of the virus with the cell membrane, the ss(-) RNA genome is released and transcribed into positive-stranded messenger RNA (mRNA) which is then translated into the viral proteins. For virus replication, a positive-stranded antigenome is produced that serves as a template for viral ss(-)RNA genome. Transcription and replication occur in the cytoplasm. Viral structure proteins and RNPs containing RNA genome assemble together and leave the infected cell by budding. $\mathrm{M}$ : matrix protein; $\mathrm{F}$ : fusion protein; $\mathrm{HN}$ : hemagglutinin-neuraminidase; $\mathrm{H}$ : hemagglutinin; $\mathrm{G}$ : glycoprotein; N or NP: nucleocapsid protein; P: phosphoprotein; L: large protein: V: protein V; C: cellular protein; SH: strongly hydrophobic protein (Harrison, Sakaguchi, and Schmitt, 2010) 


\subsubsection{The Tupaia paramyxovirus}

The Tupaia paramyxovirus (TPMV) was first isolated in 1999 from spontaneously degenerating primary kidney cells of an apparently healthy Southeast Asian tree shrew (Tupaia belangeri) (Tidona et al., 1999). Tree shrews are related to insectivores and primates, but are classified as a separate mammalian order (Scandentia). These animals are phylogenetically more closely related to primates and humans than any other laboratory animal. This makes them very interesting as an animal model for human diseases caused by viral infections (Darai et al., 1978; Xie et al., 1998).

The host range of TPMV is restricted to Tupaia cells, it does not grow in any other cell line tested so far. The receptor for cell entry of the virus is not known. Infection of Tupaia cells with TPMV results in a cytopathic effect including multinucleated syncytia followed by cell lysis. Viruses released in the supernatant of infected cells reach titers around $10^{5} \mathrm{PFU} / \mathrm{ml}$. There is no serological cross-reactivity detected between TPMV and other paramyxoviruses including measles virus, suggesting that TPMV is antigenetically distinct from these viruses.

The TPMV genome shows the typical paramyxovirus gene order N-P/C//V-M-F-H-L (Fig. 9) whereas all genes are separated by the nontranscribed trinucleotide CUU. The complete genome consists of 17,904 nucleotides which is larger than those of most paramyxoviruses (Springfeld et al., 2005).

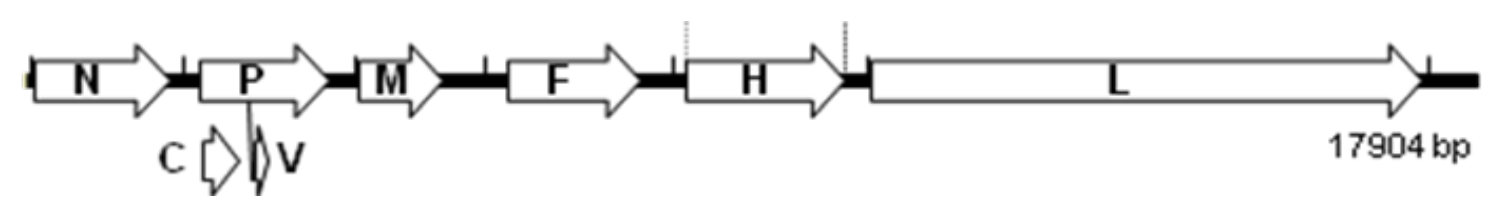

Figure 9: Gene order of the Tupaia paramyxovirus

The 17,904-nucleotide single-stranded TPMV antigenome is represented by the black bar. The arrows indicate the positions of the open reading frames (ORFs) of the N, P, C, V, M, F, H and L protein. The small vertical bars mark the positions of the intergenic sequences. (Springfeld et al., 2005)

The six genes of the TPMV genome are flanked by non-coding sequences, a control region called leader at the 3' end and a sequence known as trailer at the 5' end (Lamb, 2007).In general, for paramyxoviruses applies that the $\mathrm{N}$ gene encodes the nucleocapsid protein, which is the first gene transcribed from the viral genome and which interacts with viral RNA. The $\mathrm{P}$ gene contains overlapping open reading frames (ORFs) and encodes the proteins $P$ (phosphoprotein), $V$ and $C$. The phosphoprotein has an essential role in RNA-polymerase activity and interacts with 
the $\mathrm{L}$ and $\mathrm{N}$ proteins. The $\mathrm{V}$ and $\mathrm{C}$ proteins are accessory proteins that probably counteract host antiviral responses (Goodbourn and Randall, 2009; Ramachandran and Horvath, 2009).

The $L$ (large) gene encodes the viral RNA-dependent RNA-polymerase, which binds to the $\mathrm{N}$ and $\mathrm{P}$ proteins to from ribonucleoproteins (RNPs). The matrix (M) protein encoded by the $M$ gene is associated with the nucleocapsid, interacts with the cytoplasmic tails of the glycoproteins $\mathrm{F}$ and $\mathrm{H}$ and initiates virus assembly and budding. The glycoproteins encoded by the genes $F$ and $H$ are part of the viral envelope and mediate attachment and fusion to the target cell. Because of their essential role in this thesis they are described in more detail later on.

Analysis of the nucleotide sequence of the $\mathrm{N}$ and $\mathrm{P}$ gene revealed typical characteristics of the subfamily Paramyxovririnae and provided sufficient evidence that TPMV is phylogenetically related to the Henipaviridae and the Morbiliviridae. But since there are only low amino acid sequence homologies among TPMV, Henipaviridae and other known paramyxoviruses, there is the assumption that TPMV represents a new genus within the Paramyxovirinae (Fig. 10) (Tidona et al., 1999).

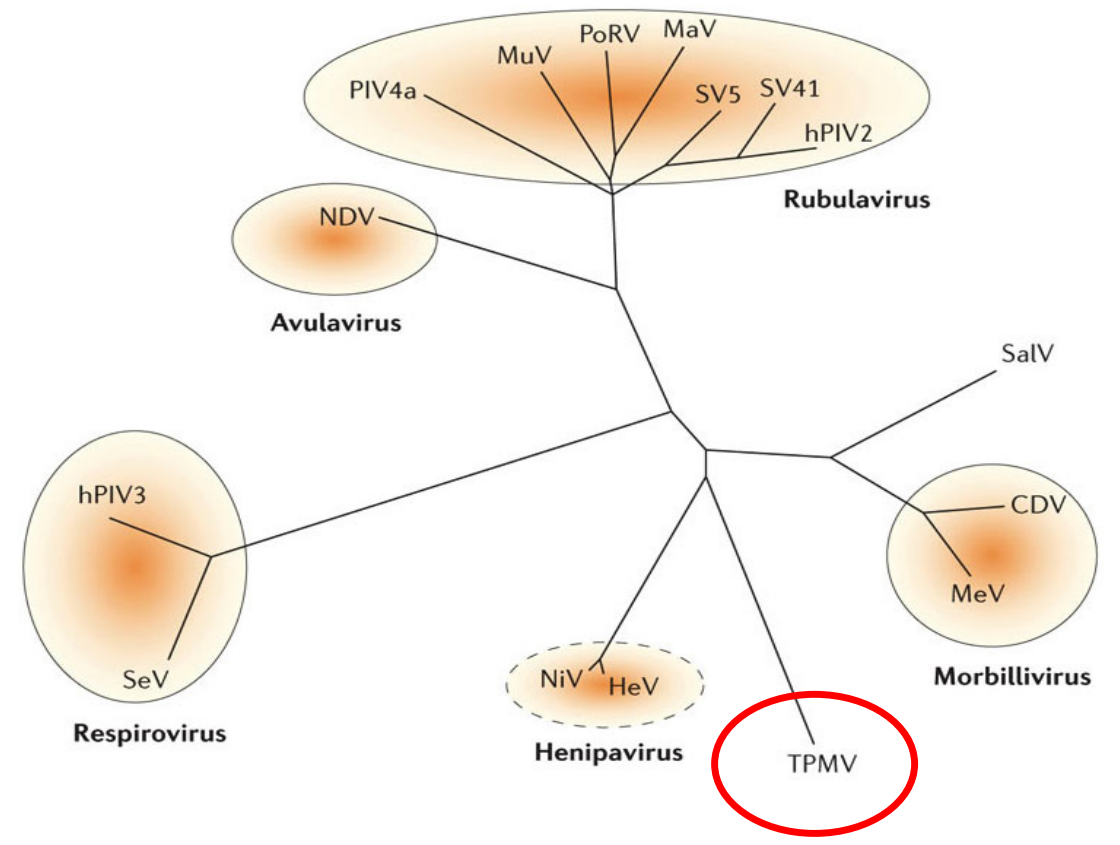

Figure 10: Phylogenetic tree of Paramyxovirinae subfamily members

Relationship of Paramyxovirinae subfamily members is based on the alignment of the amino-acid sequence of the $\mathrm{N}$ gene of selected Paramyxovirinae subfamily members. Morbillivirus genus: $\mathrm{MeV}$ (measles virus), CDV (canine distemper virus); Henipavirus genus: HeV (Hendra virus), NiV (Nipah virus); Respirovirus genus: SeV (Sendai virus), hPIV3 (human parainfluenza virus 3); Avulavirus genus: NDV (Newcastle disease virus); Rubulavirus genus: hPIV2 (human parainfluenza virus 2), MaV (Mapuera virus), MuV (mumps virus), PIV4a (parainfluenza virus 4a), PoRV (porcine rubulavirus), SV5 (simian parainfluenza virus 5), SV41 (simian parainfluenza virus 41); and unclassified viruses SalV (Salem virus) and TPMV (Tupaia paramyxovirus). (modified from (Eaton et al., 2006)) 


\subsubsection{Glycoproteins of the Tupaia paramyxovirus}

As already mentioned before, the glycoproteins of TPMV, namely $F$ and $H$, are responsible for attachment and fusion of the virus with the target cell. The $\mathrm{H}$ protein is a type II transmembrane protein with a length of 665 amino acids. Due to the ability of TPMV virions to hemagglutinate Tupaia erythrocytes the protein is named hemagglutinin $(\mathrm{H})$. It has no neuraminidase activity (Tidona, 1999). The protein is glycosylated at three $\mathrm{N}$ glycosylation sites and the corresponding molecular mass is $\sim 80 \mathrm{kDa}$ (Springfeld et al., 2005). Compared to other paramyxovirus attachment proteins, there is only low sequence identity $(<20 \%)$ and the cytoplasmic tail of TPMV $\mathrm{H}$ is unusually long (94 amino acids). The TPMV $\mathrm{H}$ protein mediates attachment of the virus to the cell surface through interactions with an unknown receptor.

A few years ago, it was shown that the TPMV $\mathrm{H}$ protein can be targeted to a designated cell type by displaying a single chain antibody ( $\mathrm{scAb}$ ) on the ectodomain (Springfeld et al., 2005), in a similar way as it had previously been shown for measles virus $\mathrm{H}$ protein (Schneider et al., 2000). Hence, it is possible to target TPMV $\mathrm{H}$ to non-Tupaia cells via respective ligands.

In contrast to TPMV $\mathrm{H}$, the $\mathrm{F}$ protein is a trimeric type I transmembrane protein that mediates fusion of the virus with the host cell membrane. It is expressed as a F0 precursor protein with a length of 553 amino acids including a hydrophobic region, the signal peptide at the N-terminus. During virus maturation and after removal of the signal peptide, the F0 protein is cleaved by an unknown protease into an N-terminal F2 and a C-terminal F1 fragment. The last one contains two hydrophobic regions, a fusion peptide at the amino terminus and the transmembrane domain. Furthermore, the TPMV F protein contains four $\mathrm{N}$ glycosylation sites in the ectodomain, three on the $\mathrm{F} 2$ fragment and one on the $\mathrm{F} 1$ fragment. As mentioned for TPMV $\mathrm{H}$, also for TPMV $F$ the similarity to fusion proteins of other paramyxoviruses is low $(33.4 \%$ identity to MV, $31.8 \%$ identity to Nipahvirus).

Fusion of paramyxoviruses occurs at the host cell membrane, it does not require the low $\mathrm{pH}$ of the endosome to trigger fusion (Lamb, 2007). In most paramyxoviruses, the attachment protein interacts with the receptor and this interaction initiates conformational changes in the $F$ protein, resulting in activation of the membrane fusion (Lamb and Jardetzky, 2007). When Tupaia cells are transfected with plasmids encoding TPMV $\mathrm{H}$ and $\mathrm{F}$, the cells fuse and form large syncytia, demonstrating that 
no other virus proteins are necessary for attachment and fusion of target cells. This observation and the possibility to retarget the $\mathrm{H}$ protein in a similar way to $\mathrm{MVH}$ make the TPMV glycoproteins very interesting for pseudotyping lentiviral vectors. Additional advantage compared to MV glycoproteins (described in section 1.3) is that it is not necessary to mutate the contact residues of TPMV $\mathrm{H}$ since it does not recognize a receptor on human cells. Because TPMV glycoproteins are derived from an animal paramyxovirus, no neutralizing antibodies in the general population are expected. 


\subsection{Aim of the study}

The aim of this thesis was to generate and characterize lentiviral vectors that are pseudotyped with the glycoproteins of the Tupaia paramyxovirus (TPMV).

Based on previously reported work for pseudotyping lentiviral vectors with truncated retargeted MV glycoproteins it was hypothesized that this system could be transferred to use TPMV glycoproteins for pseudotyping. It was previously shown that the TPMV glycoproteins can be targeted to normally non-permissive cells by displaying single-chain antibodies on the $\mathrm{H}$ protein that recognize cell surface antigens on target cells. Based on this work, the TPMV glycoproteins $\mathrm{H}$ and $\mathrm{F}$ had to be further characterized and cytoplasmic tail truncation mutants of $\mathrm{H}$ and $\mathrm{F}$ had to be screened. Additionally, in the same step, the retargeting possibility of the $\mathrm{H}$ protein had to be further investigated by displaying a single chain antibody against the $B$ cell surface marker CD20 on the ectodomain. The contact residues responsible for attachment of the cell do not have to be mutated, since TPMV is restricted to Tupaia cells. After characterization, glycoprotein mutants that support incorporation into lentiviral vectors had to be identified for vector production. Furthermore, the produced TPMV-pseudotyped vectors had to be characterized in terms of glycoprotein incorporation, their ability to mediate gene transfer and gene expression and their stability. To increase viral titers, different vector concentration techniques had to be tested. In a next step, it was necessary to investigate, if the retargeted TPMVpseudotyped vectors were able to discriminate between target and non-target cells, first on different separated receptor-positive and -negative cell lines, then in a mixed cell population. Finally, the efficiency of CD20-retargeted vector particles to transduce primary human $B$ cells needed to be examined, both for activated and quiescent primary $B$ cells.

Vectors pseudotyped with TPMV-glycoproteins would represent a major advance in gene therapy. Recently, retargeted vectors pseudotyped with MV glycoproteins were developed that allow specific cell entry to a certain cell type and transduction of quiescent primary human B cells. The presence of neutralizing antibodies in the general population due to MV vaccination or disease might limit the clinical use of these vectors. With TPMV-pseudotyped vectors, this problem can possibly be overcome as they might not be neutralized by pre-existing antibodies. 


\section{MATERIAL}

\subsection{Instruments}

Instrument

Analytical scales

Bacterial shaker

Blotting chamber

Camera (Power Shot G6)

Centrifuge (Multifuge $3 \mathrm{SR}+$ )

Centrifuge (Biofuge fresco)

Centrifuge (Biofuge pico)

Centrifuge ( $X L 70$ Ultracentrifuge)

Centrifuge (J2-HC)

CO2 incubator

Electrophoresis chamber

Electrophoresis power supply (EPS 3500)

Electrophoresis power supply (GPS 200/400)

Film cassette

Film developer

Fridge, $4^{\circ} \mathrm{C}$

Gel documentation station

Heat block

Heat block (Thermomixer Compact)

Horizontal shaker (Duomax 1030)

Hotplate / stirrer

Incubator (Function Line)

Microscope (Axiovert 40 CFL)

Microwave

Multistep pipette

Neubauer counting chamber

Nitrogen tank

\section{Distributor}

Kern, Albstadt

Infors AG, Böttmingen, $\mathrm{CH}$

Bio-Rad, Munich

Canon, Krefeld

Heraeus, Hanau

Heraeus, Hanau

Heraeus, Hanau

Beckmann, Munich

Beckmann, Munich

Heraeus, Hanau

Steinbrenner, Wiesenbach

Pharmacia Biotech, Freiburg

Pharmacia Biotech, Freiburg

Amersham, Freiburg

Amersham, Freiburg

Liebherr, Biberach

Bio-Rad, Munich

VWR, Darmstadt

Eppendorf, Hamburg

Heidolph, Kehlheim

VWR, Darmstadt

Heraeus, Hanau

Carl Zeiss, Jena

Bosch, Stuttgart

Eppendorf, Hamburg

Carl Roth $\mathrm{GmbH}$, Karlsruhe

Thermo Electron coorp., Erlangen 
$\mathrm{pH}$ meter

Photometer (Nano Drop-1,000)

Photometer

Pipettes

Scales (BL 1500 S)

Scanner Epso

SDS electrophoresis chamber

Sterile bench (Class /I Type A/B3)

Stand

Tabletop centrifuge (Mini Spin Plus)

Thermostatic water bath (WBS)

Upright freezer, $20^{\circ} \mathrm{C}$ (Comfort)

Vortex mixer

Water bath

\subsection{Consumables}

\section{Product}

Amicon 100-ultra centrifugal filter units

Centrifuge tubes $(35 \mathrm{ml})$

Cover slips

Cryo tubes $(2 \mathrm{ml})$

Cuvettes

Disposable scalpels

DNase / RNase free water

water

Filters $(0.45 \mu \mathrm{m})$

Gloves (Semper Guard Nitrile)

Gloves (Peha Soft, Latex)

HyperfilmTM ECL

Micro test tubes $(1.5 \mathrm{ml}, 2 \mathrm{ml})$

Mouth protection

Nitrocellulose membrane
WWR, Darmstadt

Peqlab Biotechnology, Erlangen

Amersham, Freiburg

Gilson, Middleton, USA

Satorius, Göttingen

Epson, Meerbusch

Bio-Rad, Munich

Sterilgard, Sanford, USA

Carl Roth $\mathrm{GmbH}$, Karlsruhe

Eppendorf, Hamburg

Fried Electric, Haifa, IL

Liebherr, Biberach

Neolab Heidelberg

Neolab, Heidelberg

\section{Distributor}

Millipore, Eschborn

Beckmann, Munich

Carl Roth $\mathrm{GmbH}$, Karlsruhe

Greiner Bio-one, Frickenhausen

Greiner, Frickenhausen

Feather, Cuome, JP

Invitrogen, Karlsruhe

Sigma, Steinheim

Whatman, Dassel

Semperit, Vienna, Austria

Paul Hartmann, Heidenheim

Amersham, Freiburg

Eppendorf, Hamburg

Meditrade, Kiefersfelden

Schleicher and Schüll, Dassel 
Parafilm

Pasteur pipettes

PCR tubes $(200 \mu \mathrm{l})$

Pipette tips (10 $\mu$ l, Filtertips)

Pipette tips (200 $\mu$ l, Filtertips)

Pipette tips (1000 $\mu$, Filtertips)

PVDF membrane

Safelock micro test tubes $(1.5 \mathrm{ml}$ and $2 \mathrm{ml})$

Serotological pipettes $(5 \mathrm{ml}, 10 \mathrm{ml}, 25 \mathrm{ml}$, $50 \mathrm{ml}$ )

Test tubes $(15 \mathrm{ml}$ and $50 \mathrm{ml})$

Tissue culture dishes $(10 \mathrm{~cm}$ and $15 \mathrm{~cm}$ )

Whatman paper

6 well tissue culture dishes

12 well tissue culture dishes

24 well tissue culture dishes

48 well tissue culture dishes

96 well tissue culture dishes

T75 cell culture flasks

T75 cell culture flasks
Pechinery Inc., Wisconsin, USA

Brand, Wertheim

Eppendorf, Hamburg

Nerbe Plus, Winsen

Nerbe Plus, Winsen

Nerbe Plus, Winsen

Bio-Rad, Munich

Eppendorf, Hamburg

BD Biosciences, San Jose, USA

Greiner Bio-one Frickenhausen

Falcon, Gräfeling-Lochham

Whatman Int., Ltd., UK

Greiner Bio-one Frickenhausen

Corning Inc., NY, USA

Greiner Bio-one Frickenhausen

Corning Inc., NY, USA

Nunc, Wiesbaden

Greiner Bio-one Frickenhausen

Greiner Bio-one Frickenhausen

\subsection{Chemicals and reagents}

\section{Chemicals}

Acetic acid (99\%)

Acrylamide bisacrylamide Solution (37.5:1)

Adenosine triphosphate (ATP)

Agarose

Ammonium persulfate (APS)

Ampicillin

Antipain

Aprotinin

Bestatin

\section{Distributor}

Sigma, Munich

Carl Roth $\mathrm{GmbH}$, Karlsruhe

Sigma, Munich

Carl Roth $\mathrm{GmbH}$, Karlsruhe

Carl Roth $\mathrm{GmbH}$, Karlsruhe

Sigma, Munich

Roche, Penzberg

Roche, Penzberg

Roche, Penzberg 
Bovine serum albumin (BSA)

Bromophenol blue

Calcium chloride

Chymostatin

Desoxynucleotides (dATP, dCTP, dGTP, dTTP)

Dipotassium phosphate

Disodium hydrogen phosphate

DMSO (Dimethyl sulfoxide)

DTT (Dithiothreitol)

Dulbecco's modified Eagle's medium (DMEM)

\section{E-64}

EDTA (Ethylenediaminetetraacetic acid), EDTA-Na 2

Ethanol (99\%)

Ethidium bromide

Fetal calf serum (FCS)

Glucose

Glycerol

Hepes

Histopaque $®-1077$

Hydrochloric acid ( $\mathrm{HCl}) 37 \%$

Igepal (Nonidet NP40)

Isopropanol

LB Agar

LB Medium

Leupeptin

L-glutamine

Lipofectamine

Pefabloc SC

Pepstatin

Phosphoramidon
Sigma, Munich

Sigma, Munich

Sigma, Munich

Roche, Penzberg

Roche, Mannheim

Applichem, Darmstadt

Sigma, Munich

Sigma, Munich

Sigma, Munich

Invitrogen, Karlsruhe

Roche, Penzberg

Sigma, Munich

Roche, Penzberg

University Hospital, Heidelberg

Carl Roth $\mathrm{GmbH}$, Karlsruhe

Invitrogen, Karsruhe

Sigma, Munich

Baker, Deventer, NL

Roth, Karlsruhe

Sigma, Munich

Acros Organics, New Jersey, USA

Sigma, Munich

Sigma, Munich

Carl Roth $\mathrm{GmbH}$, Karlsruhe

Carl Roth $\mathrm{GmbH}$, Karlsruhe

Roche, Penzberg

Biochrome AG, Berlin

Invitrogen, Karlsruhe

Roche, Penzberg

Roche, Penzberg

Roche, Penzberg 
Loading dye solution $(6 x)$

Magnesium chloride $(\mathrm{MgCl} 2)$

$\beta$ Mercaptoethanol (98\%)

Methanol (100\%)

Milk powder

Penicillin / Streptomycin (P/S)

Pepstatin

Potassium chloride $(\mathrm{KCl})$

Protamine sulfate

Proteinase inhibitor cocktail

Puromycin

Trypsin-EDTA

Sodium chloride $(\mathrm{NaCl})$

Sodium dodecyl sulfate (SDS)

Sodium hydroxide $(\mathrm{NaOH})$

N,N,N',N' Tetramethylenediamine (TEMED)

Tris base

Trypsin-EDTA solution

Tween-20

Urea

\subsection{Comercial Kits}

\section{Kit}

Dynal $\circledast$ B cell negative isolation Kit

EndoFree Plasmid Maxiprep Kit

Enhanced Chemiluminescence (ECL) Kit

Expand High Fidelity PCR System

QiaPrep Plasmid Maxiprep Kit

QiaPrep Plasmid Miniprep Kit

QiaQuick Gel Extraction

QiaQuick PCR Purification Kit
Fermentas, St. Leon Rot

Merck, Darmstadt

Sigma, Munich

Merck, Darmstadt

Carl Roth $\mathrm{GmbH}$, Karlsruhe

Invitrogen, Karlsruhe

Sigma, Munich

Sigma, Munich

Sigma, Steinbach

Roche, Penzberg

Sigma, Munich

Invitrogen, Karlsruhe

AppliChem, Darmstadt

Gerbu, Biotechnik GmbH, Gaiberg

Sigma, Munich

Carl Roth $\mathrm{GmbH}$, Karlsruhe

Sigma, Munich

Invitrogen, Karlsruhe

Sigma, Munich

Sigma, Munich

\section{Distributor}

Invitrogen, Karlsruhe

Qiagen, Hilden

Amersham Biosciences, Freiburg

Roche, Mannheim

Qiagen, Hilden

Qiagen, Hilden

Qiagen, Hilden

Qiagen, Hilden 


\subsection{Solutions and buffers}

Blocking buffer (for Western blotting)

PBS (10x)

PBS-T

RIPA lysis buffer

SDS running buffer (10x)

TAE buffer

TBS 10x
$1 \times$ PBS

$0.1 \%$ Tween 20

$5 \%$ Milk powder

$1.4 \mathrm{M} \mathrm{NaCl}$

$27 \mathrm{mM} \mathrm{KCl}$

$100 \mathrm{mM} \mathrm{Na} 2 \mathrm{HPO} 4$

$8 \mathrm{mM} \mathrm{KH} 2 \mathrm{PO} 4$

$\mathrm{pH} 6.8$

$1 x$ PBS

$0.1 \%$ Tween-20

$150 \mathrm{mM} \mathrm{NaCl}$

$50 \mathrm{mM}$ Tris- $\mathrm{HCl}, \mathrm{pH} 7.5$

$1 \%$ Nonidet P40

$0.5 \%$ Sodiumdeoxycholate

$1 \%$ sodium dodecyl sulfate

$0.25 \mathrm{M}$ Tris-base

1.92 M Glycine

$1 \%$ SDS

40mM Tris

$20 \mathrm{mM}$ acetic acid $1 \mathrm{mM}$ EDTA.

$175.3 \mathrm{~g} \mathrm{NaCl} / 2 \mathrm{I}$

$24.2 \mathrm{~g} \mathrm{Tris} / 2 \mathrm{l}$ 
TBS-T

$48.5 \mathrm{~g}$ Tris/2l, $\mathrm{pH} 7.5$

$584.4 \mathrm{~g} \mathrm{NaCl} / 2 \mathrm{l}$

$0.05 \%$ Tween-20

a.d. $\mathrm{H}_{2} \mathrm{O}$

Transfer buffer SDS-PAGE

$25 \mathrm{mM}$ Tris-base

192 mM Glycine

$20 \%$ Methanol

$0.01 \%$ SDS

6x SDS sample buffer
$0.35 \mathrm{M}$ Tris-Cl/
$0.28 \%$ SDS pH 6.8
$30 \%$ Glycerol
$10 \%$ sodium dodecyl sulfate
$9.3 \%$ DTT
0.012 \% Bromophenol Blue

All buffers were diluted in $\mathrm{H}_{2} \mathrm{O}$, unless stated otherwise

\subsection{Molecular components (enzymes, cytokines, oligonucleotides, plasmids, antibodies and sera, marker)}

\section{Enzmes}

\section{Enzyme}

restriction endonucleases

T4-DNA-ligase

High Fidelity Polymerase

\section{Distributor}

New England Biolabs, Ipswich, MA

New England Biolabs, Ipswich, MA

Roche, Mannheim 


\section{Cytokines}

\section{Cytokines}

CD40 ligand
IL-2
IL-4
IL-10

\section{Distributor}

R\&D Systems, Wiesbaden

R\&D Systems, Wiesbaden

R\&D Systems, Wiesbaden

R\&D Systems, Wiesbaden

\section{Oligonucleotides}

All oligonucleotides were synthesized from the company Eurofins MWG Operon.

\begin{tabular}{|c|c|}
\hline Primer & $5^{\prime} \rightarrow 3^{\prime}$ sequence \\
\hline HaCD20 S & GGGGCGCGCAGGTTCAGCTGGTCCAG \\
\hline TPMV-H $\Delta 20 \mathrm{~S}$ & TTTGGGGGATCCATTATGTTGCAGAGCCAGAGTG \\
\hline TPMV-H $\triangle 40 \mathrm{~S}$ & TTTGGGGGATCCATTATGTATTCAAATCCTCCAC \\
\hline TPMV-H $\Delta 50 \mathrm{~S}$ & TTTGGGGGATCCATTATGGATCAAGGTATTGATG \\
\hline TPMV-H $\Delta 60 \mathrm{~S}$ & TTTGGGGGATCCATTATGCAACCATTATCAACTC \\
\hline TPMV-H $\Delta 70 \mathrm{~S}$ & TTTGGGGGATCCATTATGCGTTACTATGGAGTTAAC \\
\hline TPMV-H $\Delta 80 \mathrm{~S}$ & TTTGGGGGATCCATTATGAGGGTGTATAATCATTTAG \\
\hline TPMV-H $\Delta 84 \mathrm{~S}$ & TTTGGGGGATCCATTATGCATTTAGGGACAATC \\
\hline TPMV-H AS & TTTGGGTCTAGATTACTTAGTATTAGGACATGTAC \\
\hline TPMV-F S & TTTGGGGGATCCCCAAGGATGGCATCACTGCTA \\
\hline TPMV-F $\Delta 8$ AS & CCCGGGTCTAGATCAATGATTCTGATGACCGTTGCTCCTGTGATG \\
\hline TPMV-F $\Delta 18$ AS & CCCGGGTCTAGATCATGATGGATTACGGAGGGAGT \\
\hline TPMV-F $\triangle 25$ AS & CCCGGGTCTAGATCAAACATAAGAATCCAGGAAATGGAGTTTGCT \\
\hline TPMV-F $\Delta 32$ AS & CCCGGGTCTAGATCAGAGTTTGCTCCATATTTTGAATGCGTAATAGC \\
\hline TPMV-FA33 AS & CCCGGGTCTAGATCATTTGCTCCATATTTTGAATGC \\
\hline TPMV-FA34 AS & CCCGGGTCTAGATCAGCTCCATATTTTGAATGCGTA \\
\hline TPMV-FA35 AS & CCCGGGTCTAGATCACCATATTTTGAATGCGTAATAGCTCACACC \\
\hline TPMV-F MV3 AS & CCGGGGTCTAGATCAACGACCTCTGAATGCGTAATAGCTCACACC \\
\hline $\begin{array}{l}\text { TPMV-Fflag } 483 \\
\text { AS }\end{array}$ & CCCGGGTCTAGATCACTTATCATCATCATCCTTATAATCCATTGA \\
\hline $\begin{array}{l}\text { TPMV-Fflag } 491 \\
\text { AS }\end{array}$ & CCCGGGTCTAGATCACTTATCATCATCATCCTTATAATCCTTATC \\
\hline $\begin{array}{l}\text { TPMV-Fflag } 492 \\
\text { AS }\end{array}$ & CCCGGGTCTAGATCACTTATCATCATCATCCTTATAATCTTTGAA \\
\hline $\begin{array}{l}\text { TPMV-Fflag } 495 \\
\text { AS }\end{array}$ & CCCGGGTCTAGATCACTTATCATCATCATCCTTATAATCCAACTT \\
\hline $\begin{array}{l}\text { TPMV-Fflag } 498 \\
\text { AS }\end{array}$ & CCCGGGTCTAGATCACTTATCATCATCATCCTTATAATCTGCTAT \\
\hline $\begin{array}{l}\text { TPMV-Fflag } 499 \\
\text { AS }\end{array}$ & $\begin{array}{l}\text { CCCGGGTCTAGATCACTTATCATCATCATCCTTATAATCAACAACAATCATT } \\
\text { GCTAT }\end{array}$ \\
\hline $\begin{array}{l}\text { TPMV-Fflag } 500 \\
\text { AS }\end{array}$ & $\begin{array}{l}\text { CCCGGGTCTAGATCACTTATCATCATCATCCTTATAATCAAAAACAACAAT } \\
\text { CATTGC }\end{array}$ \\
\hline $\begin{array}{l}\text { TPMV-Fflag } 501 \\
\text { AS }\end{array}$ & $\begin{array}{l}\text { CCCGGGTCTAGATCACTTATCATCATCATCCTTATAATCAACAATCATTGC } \\
\text { TATTAA }\end{array}$ \\
\hline
\end{tabular}




\begin{tabular}{|l|l|}
\hline TPMV-Fflag 505 & CCCGGGTCTAGATCACTTATCATCATCATCCTTATAATCACCAAAAACAAC \\
AS & AATCAT \\
\hline TPMV-Fflag 507 & CCCGGGTCTAGATCACTTATCATCATCATCCTTATAATCAAGTAGAGTACC \\
AS & AAAAAC \\
\hline TPMV-Fflag 510 & CCCGGGTCTAGATCACTTATCATCATCATCCTTATAATCGAGCCAAAGAAG \\
AS & TAGAGT \\
\hline TPMV-Fflag 513 & CCCGGGTCTAGATCACTTATCATCATCATCCTTATAATCCACACCAAAGAG \\
AS & CCAAAG \\
\hline TPMV-Fflag 516 & CCCGGGTCTAGATCACTTATCATCATCATCCTTATAATCGTAATAGCTCAC \\
AS & ACCAAA \\
\hline TPMV-Fflag 533 & CCCGGGTCTAGATCACTTATCATCATCATCCTTATAATCATTACGGAGGGA \\
AS & GTAAAC \\
\hline TPMV-Fflag 553 & CCCGGGTCTAGATCACTTATCATCATCATCCTTATAATCTCCACTTATATCT \\
AS & GTACTG \\
\hline Ala1 S & TTGTTAATAGCAATGATTGCTGCTGCTGGTACTCTACTTCTTTGG \\
\hline Ala1 AS & CCAAAGAAGTAGAGTACCAGCAGCAGCAATCATTGCTATTAACAA \\
\hline Ala2 S & TTAATAGCAATGATTGTTGCTGCTGCTACTCTACTTCTTTGGCTC \\
\hline Ala2 AS & GAGCCAAAGAAGTAGAGTAGCAGCAGCAACAATCATTGCTATTAA \\
\hline
\end{tabular}

\section{Plasmids}

\begin{tabular}{|c|c|c|}
\hline name & characterization & source \\
\hline pCG-TPMV-H & $\begin{array}{l}\text { TPMV H protein under control of } \\
\text { CMV promotor }\end{array}$ & Group Dr. Springfeld \\
\hline pCG-TPMV-HaCD20 & $\begin{array}{l}\text { TPMV H protein with aCD20- } \\
\text { scAb at the C-terminus, under } \\
\text { control of CMV promotor }\end{array}$ & Group Dr. Springfeld \\
\hline pCG-TPMV-H $\Delta 20 a C D 20$ & $\begin{array}{l}\text { HaCD20 with truncated } \\
\text { cytoplasmic tail and with aCD20- } \\
\text { scAb at the C-terminus, under } \\
\text { control of CMV promotor }\end{array}$ & this thesis \\
\hline pCG-TPMV-H $\triangle 40 a C D 20$ & $\begin{array}{l}\text { HaCD20 with truncated } \\
\text { cytoplasmic tail under control of } \\
\text { CMV promotor }\end{array}$ & this thesis \\
\hline pCG-TPMV-H $\triangle 50 a C D 20$ & $\begin{array}{l}\text { HaCD20 with truncated } \\
\text { cytoplasmic tail and with aCD20- } \\
\text { scAb at the C-terminus, under } \\
\text { control of CMV promotor }\end{array}$ & this thesis \\
\hline pCG-TPMV-H $\triangle 60 a C D 20$ & $\begin{array}{l}\text { HaCD20 with truncated } \\
\text { cytoplasmic tail and with aCD20- } \\
\text { scAb at the C-terminus,under } \\
\text { control of CMV promotor }\end{array}$ & this thesis \\
\hline pCG-TPMV-H $\triangle 70 a C D 20$ & $\begin{array}{l}\text { HaCD20 with truncated } \\
\text { cytoplasmic tail and with aCD20- } \\
\text { scAb at the C-terminus, under } \\
\text { control of CMV promotor }\end{array}$ & this thesis \\
\hline pCG-TPMV-H $\triangle 80 a C D 20$ & $\begin{array}{l}\text { HaCD20 with truncated } \\
\text { cytoplasmic and with aCD20- } \\
\text { scAb at the C-terminus, tail under } \\
\text { control of CMV promotor }\end{array}$ & this thesis \\
\hline pCG-TPMV-H $\triangle 84 \alpha C D 20$ & $\begin{array}{l}\text { HaCD20 with truncated } \\
\text { cytoplasmic tail and with aCD20- } \\
\text { scAb at the C-terminus, under }\end{array}$ & this thesis \\
\hline
\end{tabular}




\begin{tabular}{|c|c|c|}
\hline & control of CMV promotor & \\
\hline pCG-TPMV-F & $\begin{array}{l}\text { TPMV-F protein under control of } \\
\text { CMV promotor }\end{array}$ & Group Dr. Springfeld \\
\hline pCG-TPMV-F $\Delta 8$ & $\begin{array}{l}\text { TPMV F with truncated } \\
\text { cytoplasmic tail under control of } \\
\text { CMV promotor }\end{array}$ & this thesis \\
\hline pCG-TPMV-F $\Delta 18$ & $\begin{array}{l}\text { TPMV F with truncated } \\
\text { cytoplasmic tail under control of } \\
\text { CMV promotor }\end{array}$ & this thesis \\
\hline pCG-TPMV-F $\Delta 25$ & $\begin{array}{l}\text { TPMV F with truncated } \\
\text { cytoplasmic tail under control of } \\
\text { CMV promotor }\end{array}$ & this thesis \\
\hline pCG-TPMV-F $\Delta 32$ & $\begin{array}{l}\text { TPMV F with truncated } \\
\text { cytoplasmic tail under control of } \\
\text { CMV promotor }\end{array}$ & this thesis \\
\hline pCG-TPMV-F $\Delta 33$ & $\begin{array}{l}\text { TPMV F with truncated } \\
\text { cytoplasmic tail under control of } \\
\text { CMV promotor }\end{array}$ & this thesis \\
\hline pCG-TPMV-F $\Delta 34$ & $\begin{array}{l}\text { TPMV F with truncated } \\
\text { cytoplasmic tail under control of } \\
\text { CMV promotor }\end{array}$ & this thesis \\
\hline pCG-TPMV-F $\Delta 35$ & 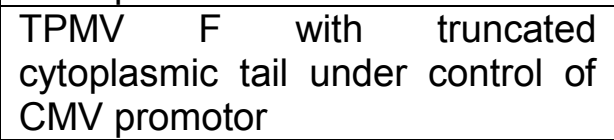 & this thesis \\
\hline pCG-TPMV-F MV3 & $\begin{array}{l}\text { TPMV F with truncated } \\
\text { cytoplasmic tail under control of } \\
\text { CMV promotor }\end{array}$ & this thesis \\
\hline pCG-TPMV-F 483 & $\begin{array}{l}\text { TPMV F protein with Flag-tag at } \\
\text { indicated amino acid position, } \\
\text { under control of CMV promotor }\end{array}$ & this thesis \\
\hline pCG-TPMV-F 491 & $\begin{array}{l}\text { TPMV F protein with Flag-tag at } \\
\text { indicated amino acid position, } \\
\text { under control of CMV promotor }\end{array}$ & this thesis \\
\hline pCG-TPMV-F 492 & $\begin{array}{l}\text { TPMV F protein with Flag-tag at } \\
\text { indicated amino acid position, } \\
\text { under control of CMV promotor }\end{array}$ & this thesis \\
\hline pCG-TPMV-F 495 & $\begin{array}{l}\text { TPMV F protein with Flag-tag at } \\
\text { indicated amino acid position, } \\
\text { under control of CMV promotor }\end{array}$ & this thesis \\
\hline pCG-TPMV-F 498 & $\begin{array}{l}\text { TPMV F protein with Flag-tag at } \\
\text { indicated amino acid position, } \\
\text { under control of CMV promotor }\end{array}$ & this thesis \\
\hline pCG-TPMV-F 499 & $\begin{array}{l}\text { TPMV F protein with Flag-tag at } \\
\text { indicated amino acid position, } \\
\text { under control of CMV promotor }\end{array}$ & this thesis \\
\hline pCG-TPMV-F 500 & $\begin{array}{l}\text { TPMV F protein with Flag-tag at } \\
\text { indicated amino acid position, } \\
\text { under control of CMV promotor }\end{array}$ & this thesis \\
\hline pCG-TPMV-F 501 & $\begin{array}{l}\text { TPMV F protein with Flag-tag at } \\
\text { indicated amino acid position, } \\
\text { under control of CMV promotor }\end{array}$ & this thesis \\
\hline pCG-TPMV-F 505 & $\begin{array}{l}\text { TPMV F protein with Flag-tag at } \\
\text { indicated amino acid position, } \\
\text { under control of CMV promotor }\end{array}$ & this thesis \\
\hline pCG-TPMV-F 507 & TPMV F protein with Flag-tag at & this thesis \\
\hline
\end{tabular}




\begin{tabular}{|c|c|c|}
\hline & $\begin{array}{l}\text { indicated amino acid position, } \\
\text { under control of CMV promotor }\end{array}$ & \\
\hline pCG-TPMV-F 510 & $\begin{array}{l}\text { TPMV F protein with Flag-tag at } \\
\text { indicated amino acid position, } \\
\text { under control of CMV promotor }\end{array}$ & this thesis \\
\hline pCG-TPMV-F 513 & $\begin{array}{l}\text { TPMV F protein with Flag-tag at } \\
\text { indicated amino acid position, } \\
\text { under control of CMV promotor }\end{array}$ & this thesis \\
\hline pCG-TPMV-F 516 & $\begin{array}{l}\text { TPMV F protein with Flag-tag at } \\
\text { indicated amino acid position, } \\
\text { under control of CMV promotor }\end{array}$ & this thesis \\
\hline pCG-TPMV-F 533 & $\begin{array}{l}\text { TPMV F protein with Flag-tag at } \\
\text { indicated amino acid position, } \\
\text { under control of CMV promotor }\end{array}$ & this thesis \\
\hline pCG-TPMV-F 553 & $\begin{array}{l}\text { TPMV F protein with Flag-tag at } \\
\text { indicated amino acid position, } \\
\text { under control of CMV promotor }\end{array}$ & this thesis \\
\hline pCG-F Ala1 & $\begin{array}{l}\text { TPMV F protein, amino acids at } \\
\text { position } 499-502 \text { replaced by } \\
\text { alanins; under control of CMV } \\
\text { promotor }\end{array}$ & this thesis \\
\hline pCG-F Ala2 & $\begin{array}{l}\text { TPMV F protein, amino acids at } \\
\text { position } 498-501 \text { replaced by } \\
\text { alanins; under control of CMV } \\
\text { promotor }\end{array}$ & this thesis \\
\hline pCG-MV ${ }_{\text {NSe }} F \Delta 30$ & $\begin{array}{l}\mathrm{MV}_{\mathrm{NSe}} \text { strain } \mathrm{F} \text { protein with a } \\
\text { truncated cytoplasmic tail; under } \\
\text { control of CMV promotor }\end{array}$ & $\begin{array}{l}\text { kindly provided by the } \\
\text { group of C.J. Buchholz, } \\
\text { Paul-Ehrlich-Institut, } \\
\text { Langen }\end{array}$ \\
\hline pCG-MV ${ }_{\text {NSe }} \mathrm{H} \Delta 18 \alpha C D 20$ & $\begin{array}{l}\mathrm{MV}_{\text {NSe }} \text { strain } \mathrm{H} \text { protein with a } \\
\text { truncated cytoplasmic tail and } \\
\text { with the four point mutations } \\
\mathrm{Y} 481 \mathrm{~A}, \mathrm{R} 533 \mathrm{~A} \text {, S548L, F549S } \\
\text { and aCD20-scAb at the C- } \\
\text { terminus; under control of CMV } \\
\text { promotor }\end{array}$ & $\begin{array}{l}\text { kindly provided by the } \\
\text { group of C.J. Buchholz, } \\
\text { Paul-Ehrlich-Institut, } \\
\text { Langen }\end{array}$ \\
\hline pCG-MV ${ }_{N S e} H \Delta 18 \alpha$ & $\begin{array}{l}\mathrm{MV}_{\text {NSe }} \text { strain } \mathrm{H} \text { protein with a } \\
\text { truncated cytoplasmic tail; under } \\
\text { control of CMV promotor }\end{array}$ & $\begin{array}{l}\text { kindly provided by the } \\
\text { group of C.J. Buchholz, } \\
\text { Paul-Ehrlich-Institut, } \\
\text { Langen }\end{array}$ \\
\hline pCG-MV ${ }_{W t} \mathrm{~F} \Delta 30$ & $\begin{array}{l}\text { MV Wildtype strain F protein with } \\
\text { a truncated cytoplasmic tail; } \\
\text { under control of CMV promotor }\end{array}$ & $\begin{array}{l}\text { kindly provided by the } \\
\text { group of C.J. Buchholz, } \\
\text { Paul-Ehrlich-Institut, } \\
\text { Langen }\end{array}$ \\
\hline pCG-MV ${ }_{W T} \mathrm{H} \Delta 18$ & $\begin{array}{l}\text { MV Wildtype strain F protein with } \\
\text { a truncated cytoplasmic tail; } \\
\text { under control of CMV promotor }\end{array}$ & $\begin{array}{l}\text { kindly provided by the } \\
\text { group of C.J. Buchholz, } \\
\text { Paul-Ehrlich-Institut, } \\
\text { Langen }\end{array}$ \\
\hline pMD.G2 & encodes VSV-G & $\begin{array}{l}\text { D. Trono, Tronolab, } \\
\text { Switzerland }\end{array}$ \\
\hline pCMV $\Delta$ R8.9 & HIV-1 packaging plasmid & $\begin{array}{l}\text { kindly provided by the } \\
\text { group of C.J. Buchholz, } \\
\text { Paul-Ehrlich-Institut, } \\
\text { Langen }\end{array}$ \\
\hline
\end{tabular}




\begin{tabular}{|l|l|l|}
\hline pSEW & $\begin{array}{l}\text { HIV-1 packagable vector } \\
\text { encoding GFP under control of } \\
\text { the SFFV promotor }\end{array}$ & $\begin{array}{l}\text { kindly provided by the } \\
\text { group of C.J. Buchholz, } \\
\text { Paul-Ehrlich-Institut, } \\
\text { Langen }\end{array}$ \\
\hline pcDI-EGFP & encodes eGFP & $\begin{array}{l}\text { kindly provided by Dr. } \\
\text { Sascha Bossow, NCT, } \\
\text { Heidelberg }\end{array}$ \\
\hline
\end{tabular}

\section{Antibodies and sera}

\begin{tabular}{|c|c|c|}
\hline name & dilution & source of supply \\
\hline $\begin{array}{l}\mathrm{aF}_{\text {ecto, }} \text { rabbit anti-TPMV } \\
\text { polyclonal peptide serum }\end{array}$ & $\begin{array}{l}\text { 1:1000 } \\
\text { western blot }\end{array}$ & PSL, Heidelberg \\
\hline $\begin{array}{l}\mathrm{aH}_{\text {ecto }} \text { rabbit anti-TPMV-H } \\
\text { polyclonal peptide serum }\end{array}$ & $\begin{array}{l}\text { 1:1000 } \\
\text { western blot }\end{array}$ & $\begin{array}{l}\text { R. Cattaneo, Mayo Clinic, } \\
\text { Rochester }\end{array}$ \\
\hline mouse anti-HIV-1 p24 mAb & $\begin{array}{l}1: 1000 \\
\text { western blot }\end{array}$ & ZeptoMetrix \\
\hline $\begin{array}{l}\text { Monoclonal Anti-Flag antibody } \\
\text { produced in mouse }\end{array}$ & $1: 1000$ & Sigma-Aldrich \\
\hline $\begin{array}{l}\text { HRP-conjugated swine-anti-rabbit } \\
\text { antibody }\end{array}$ & $\begin{array}{l}1: 2000 \\
\text { western blot }\end{array}$ & $\begin{array}{l}\text { DakoCytomation, } \\
\text { Glostrup/Denmark }\end{array}$ \\
\hline $\begin{array}{l}\text { R-Phycoerythrin (PE) conjugated } \\
\text { anti-mouse IgG (whole molecule) } \\
F\left(a b^{\prime}\right)_{2} \text { fragment }\end{array}$ & $\begin{array}{l}1: 50 \\
\text { FACS }\end{array}$ & Sigma-Aldrich \\
\hline $\begin{array}{l}\text { PE conjugated mouse anti-human } \\
\text { CD19 mAb }\end{array}$ & $\begin{array}{l}1: 10 \\
\text { FACS }\end{array}$ & $\begin{array}{l}\text { DakoCytomation, } \\
\text { Glostrup/Denmark }\end{array}$ \\
\hline $\begin{array}{l}\text { PE-Cy5, FITC or PE conjugated } \\
\text { mouse anti-human CD20 mAb }\end{array}$ & $\begin{array}{l}1: 10 \\
\text { FACS }\end{array}$ & BD Pharmingen \\
\hline $\begin{array}{l}\text { FITC-conjugated mouse anti- } \\
\text { human CD69 mAb }\end{array}$ & $\begin{array}{l}1: 10 \\
\text { FACS }\end{array}$ & $\begin{array}{l}\text { DakoCytomation, } \\
\text { Glostrup/Denmark }\end{array}$ \\
\hline $\begin{array}{l}\text { PE-Cy5, FITC or PE conjugated } \\
\text { mouse lgG }{ }_{2 b_{k}} \text { isotype control }\end{array}$ & $\begin{array}{l}1: 10 \\
\text { FACS }\end{array}$ & BD Pharmingen \\
\hline $\begin{array}{l}\text { FITC or PE conjugated mouse } \\
\text { lgG }_{1 \mathrm{k}} \text { isotype control }\end{array}$ & $\begin{array}{l}1: 10 \\
\text { FACS }\end{array}$ & BD Pharmingen \\
\hline $\begin{array}{l}\mathrm{PE} \text { conjugated mouse } \operatorname{lgG}_{2 \mathrm{a}, \mathrm{k}} \\
\text { isotype control }\end{array}$ & $\begin{array}{l}1: 10 \\
\text { FACS }\end{array}$ & BD Pharmingen \\
\hline Serum $1,2,3$ & Neutralization-Assay & $\begin{array}{l}\text { kindly provided by the group } \\
\text { 'Virotherapy', } \\
\text { Heidelberg }\end{array}$ \\
\hline Serum MV-negative & Neutralization-Assay & $\begin{array}{ll}\text { kindly provided } & \text { by the } \\
\text { Universitiy } & \text { Hospital, } \\
\text { Heidelberg } & \end{array}$ \\
\hline
\end{tabular}

\section{Molecular weight markers}

\begin{tabular}{|c|c|}
\hline \multicolumn{2}{|l|}{ DNA marker } \\
\hline 100 bp DNA ladder & NEB, Ipswich, MA, USA \\
\hline $1 \mathrm{~kb}$ DNA ladder & NEB, Ipswich, MA, USA \\
\hline \multicolumn{2}{|l|}{ Protein marker } \\
\hline $\begin{array}{l}\text { Precision Plus Protein }{ }^{\mathrm{TM}} \text { Standards, Dual color, } \\
10-250 \mathrm{kDa}\end{array}$ & Bio-Rad, Munich \\
\hline
\end{tabular}




\subsection{Bacterial strains and culture media}

\begin{tabular}{|c|c|}
\hline Strain & Distributor \\
\hline E.coli BL21 Chemically Competent Cells & Stratagene, La Jolla, USA \\
\hline $\begin{array}{l}\text { E.coli TOP } 10 \text { Chemically Competent } \\
\text { Cells }\end{array}$ & Invitrogen, Karlsruhe \\
\hline \begin{tabular}{ll}
\multicolumn{2}{l}{ Luria-Bertani (LB) } \\
\multicolumn{1}{l}{ Bacto-Trypton } & $1.0 \%(\mathrm{w} / \mathrm{v})$ \\
yeast extract & $0.5 \%(\mathrm{w} / \mathrm{v})$ \\
$\mathrm{NaCl}$ & $1.0 \%(\mathrm{w} / \mathrm{v})$ \\
$\mathrm{pH} 7.0$ &
\end{tabular} & \\
\hline
\end{tabular}

\subsection{Cell lines and culture media}

\begin{tabular}{|l|l|l|}
\hline name & characterization & Distributor \\
\hline HEK-293T & $\begin{array}{l}\text { human embryonic kidney cell line } \\
\text { genetically engineered to express } \\
\text { the large T antigen }\end{array}$ & ICLC HTL04001 \\
\hline HT1080 & $\begin{array}{l}\text { human fibrosarcoma cell line } \\
\text { human fibrosarcoma cell line } \\
\text { genetically engineered to express } \\
\text { CD20 }\end{array}$ & $\begin{array}{l}\text { R. Cattaneo, Mayo Clinic, } \\
\text { USA }\end{array}$ \\
\hline HT1080-CD02 & $\begin{array}{l}\text { human Burkitt's lymphoma cell } \\
\text { line }\end{array}$ & ATCC CCL-86 \\
\hline Raji & $\begin{array}{l}\text { human chronic myelogenous } \\
\text { leukemia cell line }\end{array}$ & ATCC CCL-243 \\
\hline K-562 & $\begin{array}{l}\text { primary Tupaia baby fibroblasts } \\
\text { R. Cattaneo, Mayo Clinic, } \\
\text { USA }\end{array}$ \\
\hline TBF &
\end{tabular}

Culture medium for HEK-293T, HT1080, TBF

Dulbecco's modified Eagle medium (DMEM) supplemented with $10 \%$ FCS and 2 mM L-glutamine.

Culture medium for Raji, K-562

RPMI 1640 supplemented with $10 \%$ FCS and 2 mM L-glutamine.

\section{Culture medium for HT1080-CD20}

RPMI 1640 supplemented with $10 \%$ FCS, 2 mM L-glutamine and $3 \mu \mathrm{g} / \mathrm{ml}$ puromycin. 


\subsection{Software}

\begin{tabular}{|l|l|}
\hline Software & Distributor \\
\hline Endnote & Thomson, Carlsbad, USA \\
\hline Microsoft Office & Microsoft, Unterschleißheim \\
\hline Photoshop & Adobe, San Jose, USA \\
\hline Pubmed & NIH \\
\hline Vector NTI Advance ${ }^{\text {TM }}$ Software & Invitrogen, Karlsruhe \\
\hline FCS-Express V3 & DeNovoSoftware, Los Angeles, USA \\
\hline
\end{tabular}




\section{METHODS}

\subsection{Molecular Biology}

\subsubsection{Agarose gel electrophoresis}

Agarose gel electrophoresis was used to analyze DNA molecules such as PCR products and digested plasmids, since it separates the fragments by their size. For production of gels, $1 \%$ agarose was dissolved in TAE buffer by heating the emulsion in a microwave until it boiled. Upon cooling, $5 \mu \mathrm{g} / \mathrm{ml}$ ethidium bromide was added. DNA samples were mixed with $8 \mu \mathrm{l}$ sample buffer and applied to the gel. Gels were run at $100 \mathrm{~V}$ for approximately $60 \mathrm{~min}$. Subsequently, DNA fragments were photographed under a UV lamp or isolated from the gel.

\subsubsection{Isolation of DNA from agarose gels}

DNA fragments were cut out of the gel under a UV lamp using a scalpel, transferred into a $1.5 \mathrm{ml}$ reaction tube and purified using the QIAquick Gel Extraction Kit, according to the manufacturer's instructions.

\subsubsection{Restriction analysis of DNA fragments or plasmids}

For restriction analysis, restriction enzymes from New England Biolabs (NEB) were used according to the manufacturer's instructions. DNA was incubated with 10-20 units/ $\mathrm{gg}$ of the respective enzyme, the corresponding buffers and, if necessary, with 0.1 volume of BSA. Reactions were incubated at the temperature optimum of the enzyme for 2-4 hours. Restriction samples were then applied to agarose gel electrophoresis.

\subsubsection{Polymerase chain reaction (PCR)}

PCR was performed to amplify DNA fragments for further molecular biological methods using a thermally stable DNA-dependent DNA-polymerase. For a typical PCR, the following ingredients in their appropriate amounts are necessary: 


\begin{tabular}{|l|l|l|}
\hline Ingredients & Amount & $\begin{array}{l}\text { Final } \\
\text { concentration }\end{array}$ \\
\hline $10 x$ polymerase buffer & $5 \mu \mathrm{l}$ & $1 \mathrm{x}$ \\
\hline $10 \mathrm{mM}$ deoxynucleotide mix (dNTP) & $1 \mu \mathrm{l}$ & $0.2 \mathrm{mM}$ \\
\hline Forward primer $(100 \mu \mathrm{M})$ & $1 \mu \mathrm{l}$ & $2 \mu \mathrm{M}$ \\
\hline Reverse primer $(100 \mu \mathrm{M})$ & $1 \mu \mathrm{l}$ & $2 \mu \mathrm{M}$ \\
\hline DNA template & $\sim 5 \mathrm{ng}$ & \\
\hline High Fidelity Polymerase & $1 \mu \mathrm{l}$ & $3.5 \mathrm{U}$ \\
\hline Double distilled water & Ad $50 \mu \mathrm{l}$ & \\
\hline
\end{tabular}

A typical PCR reaction was programmed as followed:

\begin{tabular}{|l|l|l|}
\hline Step & Temperature & Time \\
\hline Denaturation & $94^{\circ} \mathrm{C}$ & $2 \mathrm{~min}$ \\
\hline Denaturation & $94^{\circ} \mathrm{C}$ & $15 \mathrm{sec}$ \\
\hline Primer annealing & $55^{\circ} \mathrm{C}$ & $30 \mathrm{sec}$ \\
\hline Extension & $72^{\circ} \mathrm{C}$ & $90 \mathrm{sec}$ \\
\hline Extension & $72^{\circ} \mathrm{C}$ & $7 \mathrm{~min}$ \\
\hline Hold & $4^{\circ} \mathrm{C}$ & \\
\hline
\end{tabular}

Depending on the length and G-C content of the used primers, different annealing temperatures were chosen using the following formula: $\left(T_{A n}=69^{\circ} \mathrm{C}+0.41 \times(\% G C)-\right.$ 650 / bp).

Cloning of the unmodified TPMV $\mathrm{H}$ and $\mathrm{F}$ protein has been described previously (Springfeld et al., 2005). The expression plasmid pCG-TPMV-HaCD20 that encodes a fusion protein of TPMV $\mathrm{H}$ and a single-chain antibody ( $\mathrm{scAb}$ ) to human CD20 was generated by amplification of the open reading frame of the $a C D 20-s c A b$ from the plasmid pCGHaCD20 (Bucheit et al., 2003) using the forward primer 5'GGGGCGCGCAGGTTCAGCTGGTCCAG-3' that inserts a BssHII site to the 3 ' end of the $\mathrm{H}$ open reading frame and a reverse primer in the backbone of the $\mathrm{PCG}$ vector. After cutting with Bssll and Xbal, the fragment was cloned into the plasmid pCGTPMV-HXaCEA (Springfeld et al., 2005), thereby exchanging the aCEA-scAb against the aCD20-scAb. TPMV H cytoplasmic tail (CT) variants were generated by PCRcloning using primers that deleted the desired amino acids and introduced a new start codon. The TPMV F CT variants and $F_{\text {Flag }}$ variants were constructed using primers that introduced a stop codon and a flag-tag at the desired position, 
respectively, and deleted the following amino acids. The TPMV-F MV3 expression plasmid was generated using a primer that introduced the three cytoplasmic tail amino acids (arginine - glycine - arginine) followed by a stop codon.

All PCR reactions were performed using an PCR Express, Therm Hybai cycler. Subsequently, they were analyzed by agarose gel electrophoresis or frozen at $-20^{\circ} \mathrm{C}$.

\subsubsection{Ligation}

For Ligation, 50-200 ng of vector DNA was used for each reaction. The ratio of insert to vector DNA was 3:1. Vector and insert DNA were mixed in a volume of $10 \mu \mathrm{l}$ and incubated at $45^{\circ} \mathrm{C}$ for $5 \mathrm{~min}$. Afterwards ligation buffer and T4 DNA ligase were added and the reaction was incubated at room temperature for 2-5 hours. The ligated DNA was used immediately for transformation of bacteria or stored at $-20^{\circ} \mathrm{C}$.

\subsubsection{Transformation of bacteria}

For transformation, chemically competent $E$. Coli bacteria were thawed on ice and 1 $\mu \mathrm{LNA}$ or for ligations, the whole ligation reaction were added and incubated on ice for 20 min. Afterwards, a heat shock of $42^{\circ} \mathrm{C}$ was performed for $45 \mathrm{sec}$ in a water bath following 5 min further incubation on ice. Then, $500 \mu \mathrm{l}$ LB medium (RT) were added, followed by incubation at $600 \mathrm{rpm}, 37^{\circ} \mathrm{C}$ for $60 \mathrm{~min}$ in a thermoblock. The suspension was added on LB plates containing ampicillin and incubated over night at $4^{\circ} \mathrm{C}$.

\subsubsection{Cultivation of bacteria}

Culture plates: Bacteria were cultured on LB plates containing ampicillin $(50 \mu \mathrm{g} / \mathrm{ml}$, $L_{\mathrm{Amp}}$ ) by adding the bacterial suspension onto the plate and incubation overnight at $37^{\circ} \mathrm{C}$ until bacterial colonies were visible. Plates with bacterial colonies were stored up to 6 weeks at $4^{\circ} \mathrm{C}$.

Liquid culture: Bacteria were cultured in liquid LB medium containing ampicillin $(0.1$ $\mathrm{mg} / \mathrm{ml}, \mathrm{LB}_{\mathrm{Amp}}$ ) overnight at $37^{\circ} \mathrm{C}$ on a shaker ( $\left.200 \mathrm{rpm}\right)$. 


\subsubsection{Plasmid preparation}

For plasmid preparation from the transformed bacteria, either the QIAprep® Spin Miniprep kit or the EndoFree ${ }^{\circledR}$ Plasmid Maxi kit was used according to the instructions of the manufacturer (Quiagen).

For extractions of small amounts of DNA (Miniprep), $5 \mathrm{ml} \mathrm{LB}$ Amp were inoculated with bacteria and incubated overnight at $37^{\circ} \mathrm{C}$. The next day, $1.5 \mathrm{ml}$ of the bacterial suspension were applied to a $1.5 \mathrm{ml}$ reaction tube and centrifuged in for $3 \mathrm{~min}$ at $5000 \mathrm{rpm}$ in a table centrifuge (RT). This step was repeated three times in the same reaction tube. DNA extraction out of the resulting bacterial pellet was performed with the the QIAprep $®$ Spin Miniprep kit.

For extraction of large amounts of DNA (Maxiprep), 1.0 I LB Lmp $_{\text {Amp }}$ were inoculated with bacteria and cultivated overnight at $37^{\circ} \mathrm{C}$. The bacteria suspension was centrifuged for $15 \mathrm{~min}, 4^{\circ} \mathrm{C}$ and $6000 \mathrm{rpm}$ (J2-HC, Beckman) and the resulting pellet was treated with the EndoFree $\AA$ Plasmid Maxi kit according to the manufacturer's instructions.

The concentrations of the isolated plasmid DNA was determined photometrically (absorption $260 \mathrm{~nm}$ ).

\subsubsection{DNA sequencing}

Plasmids were sequenced by the company GATC. Samples were prepared in a volume of $30 \mu \mathrm{l}$ containing $50 \mathrm{ng} / \mu \mathrm{l}$ DNA and send together with $30 \mu \mathrm{l}$ of appropriate primers $(10 \mathrm{pmol} / \mu \mathrm{l})$ via mail to the company.

\subsection{Cell Biology and virology}

\subsubsection{Cultivation of cell lines}

Cells were cultivated in an incubator at $37^{\circ} \mathrm{C}, 5 \% \mathrm{CO}_{2}$ and saturated water atmosphere. They were passaged twice a week by removing the medium, washing with PBS and adding trypsin. After 1-2 minutes, fresh medium was added to the cells and trypsin. Out of this solution, an appropriate fraction was seeded into a new culture flask with fresh medium. 


\subsubsection{Freezing and thawing of cells}

For freezing, cells were trypsinised and resuspended in medium as described in section 2.2.1. This cell solution was then centrifuged at $900 \mathrm{rpm}$ for $4 \mathrm{~min}$ at $4^{\circ} \mathrm{C}$ (Multifuge $3 \mathrm{SR}+$ ), the pellet resuspended in cold freezing medium $\left(4^{\circ} \mathrm{C}, 70 \% \mathrm{FCS}\right.$, $15 \%$ DMSO, $15 \%$ DMEM or RPMI) and aliquoted in $1 \mathrm{ml}$ fractions containing approximately $1 \times 10^{6}$ cells. Afterwards, the aliquots were stored for $24 \mathrm{~h}$ at $-20^{\circ} \mathrm{C}$, then for $24 \mathrm{~h}$ at $-80^{\circ} \mathrm{C}$ and finally transferred into liquid nitrogen.

Cells were thawed by putting the cryotube into a waterbath $\left(37^{\circ} \mathrm{C}\right)$ until the cell suspension is liquid. Then, the cells were immediately transferred into a $15 \mathrm{ml}$ reaction tube containing pre-warmed $\left(37^{\circ}\right)$ fresh medium. To exclude the toxic DMSO, the cells were centrifuged at $900 \mathrm{rpm}$ for $3 \mathrm{~min}$ at RT (Multifuge $3 \mathrm{SR}+$ ) and the pellet resuspended in $10 \mathrm{ml}$ fresh medium which was then transferred into a cell culture flask.

\subsubsection{Transient transfection of cells}

293T cells, HT1080-CD20 cells and TBF cells were transient transfected using Lipofectamine. Twenty-four hours before transfection, cells were seeded as suggested by the manufacturer. One hour before transfection, medium was replaced by fresh medium. The plasmids were mixed with Lipofectamine and added to the cells. Fourty-eight hours after transfection, the cells were analyzed.

\subsubsection{Isolation of human peripheral blood mononuclear cells (PBMCs)}

PBMCs were isolated from human blood from Buffy coats (Blutspendedienst Hessen, Frankfurt a.M.) using the method of density centrifugation with Histopaque $®-1077.15$ $\mathrm{ml}$ of cold Histopaque $\left(4^{\circ} \mathrm{C}\right)$ were overlaid with $25 \mathrm{ml}$ of a $1: 1$ mixture of human blood and PBS in a $50 \mathrm{ml}$ Falcon tube. The mixture was centrifuged at 1,800 rpm for $30 \mathrm{~min}$ (RT, no break, Multifuge $3 \mathrm{SR}+$ ) to pellet the red blood cells and granulocytes. After centrifugation, a small white fraction is visible above the Histopaque that contains lymphocytes, monocytes and macrophages. Above this fraction, the plasma is present. The white lymphocyte-containing fraction was collected into a $50 \mathrm{ml}$ Falcon ( $5 \mathrm{ml}$ per Falcon). Then, $50 \mathrm{ml}$ PBS were added to each Flacon tube followed by centrifugation at $1,500 \mathrm{rpm}$ for $10 \mathrm{~min}$ at RT. The pellets were combined in one single Falcon tube and washed against with PBS as described before. Remaining 
erythrocytes were lysed by adding $10 \mathrm{ml} 0.86 \%$ ammonium chloride and incubation for $20 \mathrm{~min}$ at $37^{\circ} \mathrm{C}$. Again, the cells were washed twice with PBS as described before and resuspended in $50 \mathrm{ml}$ PBS for determination of the cell number. Subsequent, the PBMCs were applied for $B$ cell isolation.

\subsubsection{Isolation and activation of primary human $B$ cells}

Primary human $B$ cells were isolated from fresh isolated PBMCs using the Dynal $B$ cell negative isolation kit (Invitrogen) as suggested by the manufacturer instructions. Isolated B cells were either used immediately for transduction or activated for 48 hours in medium together with $300 \mathrm{ng} / \mathrm{ml}$ CD40 ligand, $50 \mathrm{ng} / \mathrm{ml}$ interleukin-2, 10 $\mathrm{ng} / \mathrm{ml}$ interleukin-4 and $10 \mathrm{ng} / \mathrm{ml}$ interleukin-10. Stimulated B cells were detected by an antibody against the activation marker CD69.

\subsubsection{Generation and concentration of lentiviral vectors}

For screening of all $\mathrm{F}$ - and $\mathrm{H}$-combinations, vector particles were generated by transfection of 293T cells using polyethylene-imine (PEI) with $1.73 \mu \mathrm{g}$ of the lentiviral transfer vector plasmid pSEW (Miyoshi et al., 1997), $1.65 \mu \mathrm{g}$ of the packaging plasmid pCMVAR8.9 (Zufferey et al., 1997) and $0.27 \mu \mathrm{g}$ each of the TPMV-H- and Fprotein variant expression plasmids. Twenty-four hours before transfection, $2.0 \times 10^{6}$ cells/well were seeded into a single well of a six-well plate. On the day of transfection, the medium was replaced by $0.5 \mathrm{ml}$ DMEM ( $+1 \%$ glutamine). The plasmids were mixed with $17.7 \mu \mathrm{l}$ of $5 \%$ (wt/vol) glucose. In a different tube, $17.7 \mu \mathrm{l}$ of $5 \%$ glucose was mixed with $4 \mu \mathrm{l}$ of $18 \mathrm{mM} \mathrm{PEI}$. Both solutions were incubated for 10 minutes at room temperature, then combined, mixed and incubated again at room temperature. After 10 minutes, the volume of the mixture was increased with $1 \mathrm{ml}$ of DMEM ( $+1 \%$ glutamine) and then added to the cells. Finally, $100 \mu$ l of FCS per well was added to the cells 5-6 hours after transfection. Forty-eight hours afterwards, the cell supernatant containing the viral particles was collected and $150 \mu \mathrm{l}$ were directly used for transduction. The resulting vectors were titrated on Raji cells. For measles virus pseudotyped vectors, $0.15 \mu \mathrm{g} \mathrm{H}$-plasmid and $0.46 \mu \mathrm{g}$ F-plasmid were used, for VSV-G-pseudotyped vectors, $0.6 \mu \mathrm{g}$ of pMD.G2 (Didier Trono, Tronolab, Lausanne, Switzerland). 
For all other experiments, vector stocks were produced by transfection using $70 \mu \mathrm{l}$ $\mathrm{PEI}$ and $310 \mu \mathrm{l}$ glucose for a T175 flask. One day before transfection, $1.5 \times 10^{7} 293 \mathrm{~T}$ cells were seeded and transfected with $28.7 \mu \mathrm{g}$ of packaging plasmid, $30.2 \mu \mathrm{g}$ of transfer vector plasmid and $5.3 \mu \mathrm{g}$ each of TPMV-H and F-expression plasmids. The measles virus $\mathrm{H}$ protein expression plasmid ${ }^{4}$ was used in an amount of $2.6 \mu \mathrm{g}$, the measles virus $\mathrm{F}$ protein plasmid in an amount of $8 \mu \mathrm{g}$. For VSV-G-pseudotyped vectors, $10.6 \mu \mathrm{g}$ pMD.G2 plasmid was used. To the transfection mixture $2.2 \mathrm{ml}$ DMEM $(+1 \%$ glutamine) was added. For concentrating the vector particles, the supernatant was collected after 48 hours, filtered $(0.45 \mu \mathrm{m}$ filter) and centrifuged by low-speed centrifugation over a sucrose-cushion (20\% wt/vol) (4000 g for 24 hours at $4{ }^{\circ} \mathrm{C}$ ). The supernatant was discarded and the pellet was resuspended in $60 \mu \mathrm{l}$ Opti-MEM (200 $\mu$ l for VSV-G-pseudotyped vectors).

For stability assays, vectors were concentrated by ultracentrifugation or ultrafiltration. Ultracentrifugation was performed at $28,000 \mathrm{rpm}$ for 3 hours at $4^{\circ} \mathrm{C}$ over a sucrosecushion $(20 \% \mathrm{wt} / \mathrm{vol})$. For ultrafiltration, the vector-containing cell supernatant was applied to Amicon 100-ultra centrifugal filter units (Millipore) and centrifuged at 4000 $\mathrm{g}$ for $90 \mathrm{~min}$ at $4^{\circ} \mathrm{C}$.

\subsubsection{Titration of lentiviral vectors}

Vector titration was done using Raji cells. For the initial screening of all TPMV-F-and $\mathrm{H}$-protein combinations, $2.0 \times 10^{4}$ Raji cells were seeded on the day of transduction into a single well of a 96-well plate in a total amount of $50 \mu \mathrm{l}$. The vector stocks were serially diluted in steps of 1:10 in a total volume of $200 \mu \mathrm{l}$. Of these dilutions, $150 \mu \mathrm{l}$ were added to the cells together with $4 \mathrm{ng} / \mathrm{ml}$ protamine sulfate and incubated for 3 hours at $37^{\circ} \mathrm{C}$. Then, $100 \mu \mathrm{l}$ fresh medium was added to the cells. After 72 hours, GFP-positive cells were determined by FACS analysis and the titer was calculated. For titration of large scale productions, $1.0 \times 10^{5}$ cells (Raji) were seeded into a single well of a 48 well plate. Serial dilutions were performed in a total volume of $300 \mu \mathrm{l}$ and $250 \mu \mathrm{l}$ were added to the cells together with $4 \mathrm{ng} / \mathrm{ml}$ protamine sulfate. After 3 hours, $0.7 \mathrm{ml}$ fresh medium was added to the cells and FACS analysis was performed 72 hours after transduction. For titer calculation, dilutions were chosen in which $2-20$ $\%$ of the cells showed GFP expression. Titers (in transducing units (t.u.)/ml) were calculated using the following formular: 
Titer [t.u./ml] $=\frac{\text { Number of cells at time of transduction }}{\text { Volume of vectors }} \times \frac{\text { GFP positive cells [\%] }}{100}$

\subsubsection{Transduction of adherent and suspension cells}

One day before transduction of adherent cell lines, $5.0 \times 10^{4}$ cells were seeded into a single well of a 24 well plate. Before transduction, the medium was removed. Depending on vector titer and MOI (multiplicity of infection), vectors were diluted with medium in an appropriate ratio in a total volume of $300 \mu \mathrm{l}$ which was then added to the cells together with $4 \mathrm{ng} / \mathrm{ml}$ protamine sulfate and incubated for 3 hours at $37^{\circ} \mathrm{C}$. Then, $700 \mu$ of fresh medium was added.

For transduction of suspension cells, $1.0 \times 10^{5}$ cells were seeded on the day of transduction into a single well of a 48 well plate in a total amount of $50 \mu \mathrm{l}$. As described above, vector and medium was mixed in an appropriate ratio depending on vector titer and $\mathrm{MOI}$ and added to the cells together with $4 \mathrm{ng} / \mathrm{ml}$ protamine sulfate and incubated for 3 hours at $37^{\circ} \mathrm{C}$. Then, $700 \mu$ of fresh medium was added.

For the transduction of the mixed cell population, $5.0 \times 10^{4}$ Raji cells together with $5.0 \times 10^{4} \mathrm{~K}-562$ cells were seeded into a single well of a 48 -well plate in a total amount of $50 \mathrm{ml}$ and transduced as described above. FACS analysis was performed 72 hours after transduction.

\subsubsection{Transduction of primary human $B$ cells}

For transduction, the isolated B cells were seeded into a single well of a 48 well plate $\left(5.0 \times 10^{4}\right.$ cells/well) together with the virus particles in a total volume of $300 \mu$ and with $4 \mathrm{ng} / \mathrm{ml}$ protamine sulfate. This was followed by centrifugation at $430 \mathrm{~g}$ for 30 $\min$ at $32^{\circ} \mathrm{C}$. Subsequently, the cells were incubated at $37^{\circ} \mathrm{C}$. Next day, $500 \mu \mathrm{l} /$ well medium (as described above) was added to the cells and 72 hours after transduction, the fraction of GFP-positive cells was determined by FACS analysis.

\subsubsection{Neutralization assay}

To examine whether TPMV-pseudotyped LVs by MV-antibodies, human sera were heat-inactivated for $30 \mathrm{~min}$ at $56^{\circ} \mathrm{C}$ and stepwise diluted (1:20) in medium without FCS. $100 \mu \mathrm{l}$ each of these dilutions were incubated for $20 \mathrm{~min}$ at $4^{\circ} \mathrm{C}$ with the appropriate amount of vector stock using a $\mathrm{MOI}$ of 0.4 . Afterwards, the vector- 
containing serum dilutions were incubated for $5 \mathrm{~min}$ at $37^{\circ} \mathrm{C}$ and $2.0 \times 10^{4}$ Raji cells in an amount of $20 \mu \mathrm{l}$ medium without FCS were added. Three hours later, $100 \mu \mathrm{l}$ fresh medium (+ FCS) was added to the cells and 72 hours after transduction, GFP expression was analyzed by FACS.

\subsubsection{Cultivation and concentration of the Tupaia paramyxovirus}

TPMV was propagated in TBF cells (Tupaia baby fibroblasts). TBFs were infected when they were $80 \%$ confluent with $1 \mathrm{ml}$ frozen virus-containing cell supernatant by adding the virus to the medium. After 5-6 days, the supernatants of two T175 flasks were combined and ultra-centrifuged at $28,000 \mathrm{rpm}$ for 3 hours at $4^{\circ} \mathrm{C}$ over a $20 \%$ sucrose-cushion. The virus-containing pellet was resuspended in $200 \mu \mathrm{l}$ PBS.

\subsubsection{Fluorescent activated cell sorting (FACS)}

FACS analysis was performed on a BD LSRII flow cytometer (Becton Dickinson, Heidelberg, Germany). Raji and K-562 cells were washed twice in $500 \mu \mathrm{l}$ FACS washing buffer (phosphate-buffered saline, $1 \% \mathrm{FCS}, 0.1 \% \mathrm{NaN}_{3}$ ) and finally resuspended in $100 \mu \mathrm{l}$ phosphate-buffered saline/1\% paraformaldehyde. To detect CD20-positive cells, the cells were incubated with a 1:10 dilution of the mouse antihuman CD20/PE-Cy5 antibody (BD Pharmingen, Heidelberg, Germany) after the first washing step. After 20 minutes at $4^{\circ} \mathrm{C}$, the antibody solution was removed and the cells were washed twice and fixed as described before. For detection of CD19positive cells, the mouse anti-human CD19/PE antibody (DakoCytomation, Hamburg, Germany) was used. Stimulated B cells were detected by an antibody against the activation marker CD69 (mouse anti-human CD69/FITC, DakoCytomation, Hamburg, Germany). The data were analyzed with the FCS-Express V3 program.

\subsection{Biochemistry}

\subsubsection{SDS-Polyacrylamide gelelectrophoresis}

For analysis of protein expression levels and for separation of proteins according to their molecular weight, a sodium dodecyl sulphate polyacrylamide gel electrophoresis (SDS-PAGE) was applied (Laemmli, 1970). In this method, proteins get denatured and negatively charged in the presence of sodium dodecyl sulphate (SDS). Thus, 
they can be applied to electrophoresis and run towards the positive pole under current conduction. Additionally, exposed disulfide bonds are reduced by the presence of dithiothreitol (DTT) resulting in prevention of intra- and intermolecular interactions by disulfide bond formation.

For analysis, proteins were resuspended in a defined volume of PBS or RIPA-Lysis buffer and 5 volumes of $6 \times$ SDS sample buffer. Samples were denatured by incubation for 5 minutes at $95{ }^{\circ} \mathrm{C}$. Afterwards, the protein samples were applied to 10 $\%$ sodium dodecyl sulfate-polyacrylamide electrophoresis gels within a Bio-Rad-Mini Protean II chamber filled with SDS-running buffer. As protein ladder, Precision Plus Protein Standards Dual Color (Bio-Rad) was used. For separation of proteins inside the stacking gel a voltage of $80 \mathrm{~V}$ was used, whereas the voltage was raised to 120 $\checkmark$ inside the separating gel until the dye front had left the separating gel.

\subsubsection{Western Blot analysis}

This technique allows the transfer of proteins onto a Polyvinylidene fluoride (PVDF) membrane. Based on this transfer, proteins can be visualized by immunostaining with specific antibodies. In general, proteins are transferred onto membranes after SDS-Polyacrylamide gelelectrophoresis, a process also called blotted. In this thesis, proteins were blotted using a wet blot. To set up the blot, the membrane was shortly incubated in methanol for activation and subsequently soaked in Western Blot transfer buffer together with four Whatman filter papers and two Western Blot sponges. Blotting was performed at $100 \mathrm{~V}$ for 1 hour or at $30 \mathrm{~V}$ overnight in transfer buffer. Subsequently, the blot was incubated in $10 \%$ milk powder/TBS-T for one hour (RT) or overnight $\left(4^{\circ} \mathrm{C}\right)$ to block unspecific protein binding sites. Specific primary antibodies were diluted in $5 \%$ milk powder/TBS-T, respectively, and incubated with the membranes for 1 hour at room temperature or overnight at $4^{\circ} \mathrm{C}$. Then, the membrane was washed once with $1 \times$ TBS-T and at least three times with $1 \times$ TBS for $10 \mathrm{~min}$ followed by an one hour incubation with the secondary horse radish peroxidase (HRP)-coupled antibody directed against the first antibody at a dilution of $1: 2000$. Again, the membrane was washed as mentioned above. Detection of specific bands was carried out using the enhanced chemiluminescence system (ECLTM) Western Blotting Detection Reagent. Hyperfilm ECL films were exposed to the resulting chemiluminescent signal. Different exposure times were chosen, depending on the quality of specific antibodies. 


\subsubsection{Preparation of cell lysates}

Cells were grown in 6 wells and washed with $2 \mathrm{ml}$ ice-cold PBS. Subsequently, 200 $\mu \mathrm{I}$ RIPA-lysis buffer were added and the plate was incubated for $5 \mathrm{~min}$ on ice. After incubation, the lysate was carefully transferred into a $1.5 \mathrm{ml}$ reaction tube and centrifuged at $13,000 \mathrm{rpm}$ for $10 \mathrm{~min}$ at $4^{\circ} \mathrm{C}$ in a table centrifuge to remove the cell debris. The resulting supernatant was then transferred into a fresh $1.5 \mathrm{ml}$ reaction tube and either used immediately for Bradford assay or stored at $-20^{\circ} \mathrm{C}$.

\subsubsection{Protease inhibitor assays}

To identify the protease involved in TPMV $F$ protein processing different protease inhibitors were tested. The inhibitors were diluted as recommended by the manufacturer (Roche). Cells were transiently transfected in a 6 well plate as described before. Five hours after transfection, the medium was replaced by $2 \mathrm{ml}$ fresh medium supplemented with protease inhibitors. Cell lysates were harvested 48 hours after transfection as described above.

\subsubsection{Bradford assay}

For determination of protein concentration of cell lysates the Bradford Dye reagent from Bio-Rad was used according to the manufacturer's instructions. Absorption of protein samples was measured in a spectrophotometer at a wavelength of $595 \mathrm{~nm}$. A standard curve was determined and protein concentrations were calculated. 


\section{RESULTS}

\subsection{Generation and characterization of modified TPMV}

\section{glycoproteins}

The aim of this thesis is to develop and characterize targeted lentiviral vectors (LVs) that are pseudotyped with the Tupaia paramyxovirus (TPMV) F and $\mathrm{H}$ glycoproteins. Recently, it was reported that lentiviral vectors can be pseudotyped with the glycoproteins of measles virus (MV) when their cytoplasmic tails are truncated (Frecha et al., 2008; Funke et al., 2008a; Funke et al., 2009). Accordingly, a panel of TPMV $\mathrm{H}$ and $\mathrm{F}$ proteins with truncated cytoplasmic tails was generated and biochemically characterized. Since it was previously shown that the TPMV attachment protein $(\mathrm{H})$ can be retargeted to cell surface molecules similar as the $\mathrm{MV}$ $\mathrm{H}$ protein (Springfeld et al., 2005), a single-chain antibody (scAb) against CD20 was displayed on the ectodomain. To identify TPMV cytoplasmic tail variants that have the potential to form functional pseudotyped LVs, surface expression of the $\mathrm{H}$ protein variants and syncytia assays were performed.

\subsubsection{Modification of the TPMV H protein}

The TPMV $\mathrm{H}$ protein is a type II membrane protein with a length of 665 amino acids and has an unusually long cytoplasmic tail of 94 amino acids. It can be retargeted to cell surface molecules by displaying single-chain antibodies on the ectodomain (Springfeld et al., 2005). Since the retargeted MV-pseudotyped LVs were established with a scAb against the B cell surface marker CD20 displayed on the ectodomain of the MV $\mathrm{H}$ protein, this molecule was also chosen as a target to establish the TPMVpseudotyped vectors.

To retarget the TPMV $\mathrm{H}$ protein to CD20, the open reading frame of the corresponding scAb was amplified from the plasmid pCGH $\alpha$ CD20 (Bucheit et al., 2003) and after cutting with Bssll and Xbal, the fragment was cloned into the plasmid pCG-TPMV-HXaCEA (Springfeld et al., 2005), thereby exchanging the CEA scAb against the CD20 scAb on the carboxyterminus of the $\mathrm{H}$ protein, resulting in the plasmid pCG-TPMV-HaCD20 (Fig. 11A). 


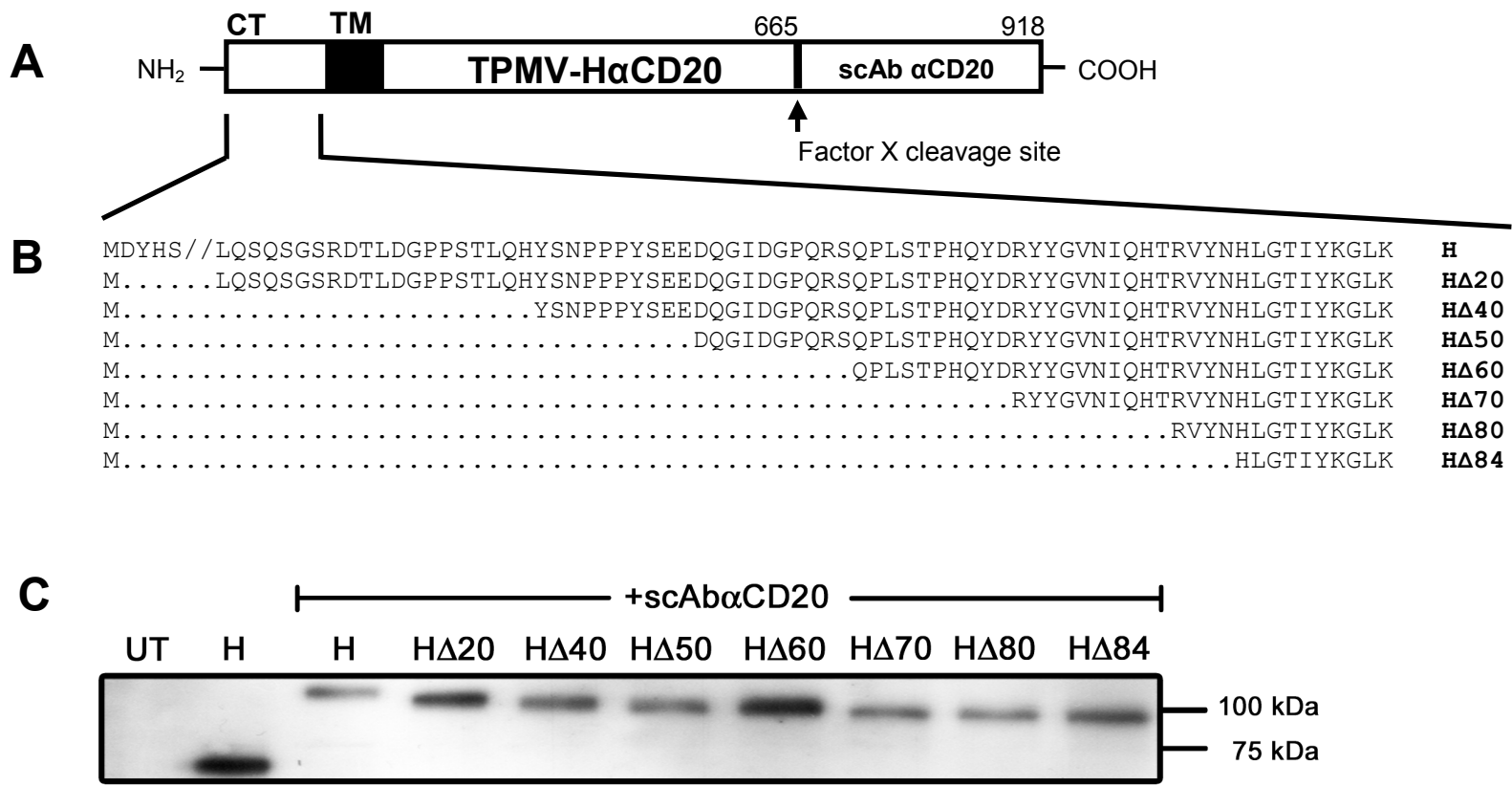

Figure 11: Schematic drawing of the TPMV H protein, amino acid sequences of the truncated cytoplasmic tails and Western blot analysis of the different $H$ protein variants

For retargeting, a single chain antibody (scAb) against CD20 was fused to the carboxyterminus of the $\mathrm{H}$ protein together with a Factor X cleavage site (A). The glycoprotein's cytoplasmic tail (CT) was truncated as indicated (B). All proteins were expressed in 293T cells, fractionated by SDS-10\% PAGE, and detected with a peptide antiserum against the ectodomain of the $\mathrm{H}$ protein $\left(\mathrm{aH}_{\text {ecto, }} \mathrm{C}\right)$

TM: transmembrane domain; UT: untransfected cells

Measles virus-pseudotyped LVs can only been generated when cytoplasmic tails of the glycoproteins are truncated. For the MV H protein, a deletion of 18 amino acids results in highest vector titers. Accordingly, a panel of TPMV $\mathrm{H}$ proteins with truncated cytoplasmic tails was generated by mutation PCR, where the numbers of deleted amino acids were randomly chosen. For the different deletions forward primers were used that are complementary until the start codon followed by an interruption and continue exactly after the deleting sequence. Additionally, the forward primers introduced a BamHI cleavage site. The reverse primer was the same for all TPMV $\mathrm{H}$ variants and introduced an Xbal cleavage site after the stop codon. As initial plasmid pCG-TPMV-HaCD20 was used. The resulting PCR fragments contained a BamHI cleavage site at the 5' end, a Xbal cleavage site at the 3' and were deleted by $20,40,50,60,70,80$ or 84 amino acids at the aminoterminus (Fig. $11, B)$, leaving only 10 aa on the shortest variant (H $\Delta 84 a C D 20)$. Subsequently, the TPMV HaCD20-coding region in the original plasmid pCG-TPMV-HaCD20 was replaced upon BamHI/Xbal digestion by the PCR fragments. 
For biochemical analysis of the different TPMV H protein variants, 293T cells were transfected with the respective plasmids and after 48 hours cell lysates were harvested, applied to SDS-polyacrylamide-gelelectrophoresis and analyzed by Western Blot using the $\mathrm{H}_{\text {ecto }}$ antibody. In repeated experiments, no relevant differences in the expression levels of the $\mathrm{H}$ protein were observed (representative Blot is shown in Fig. 11, C). Fusion of the aCD20 scAb to the carboxyterminus of the $\mathrm{H}$ protein resulted in a larger molecular mass of approximately $110 \mathrm{kDa}$ compared to the unmodified $\mathrm{H}$ protein. As expected, $\mathrm{H}$ protein size was reduced in the proteins with truncated cytoplasmic tail.

\subsubsection{Modification of the TPMV F protein}

The TPMV $F$ protein is a 553 amino acid type I membrane protein. It is expressed as a F0 precursor protein and, in addition to the removal of the signal peptide, cleaved during activation, resulting in the fragments F1 and F2 (Fig. 12, A).

A

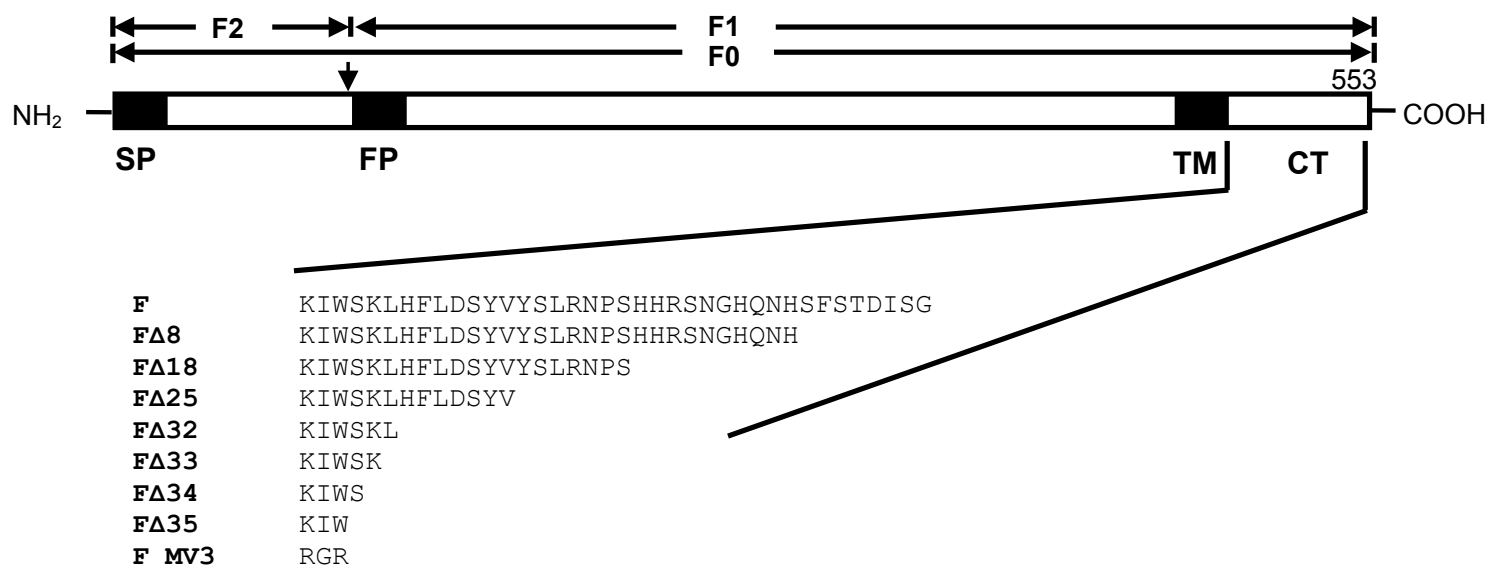

C

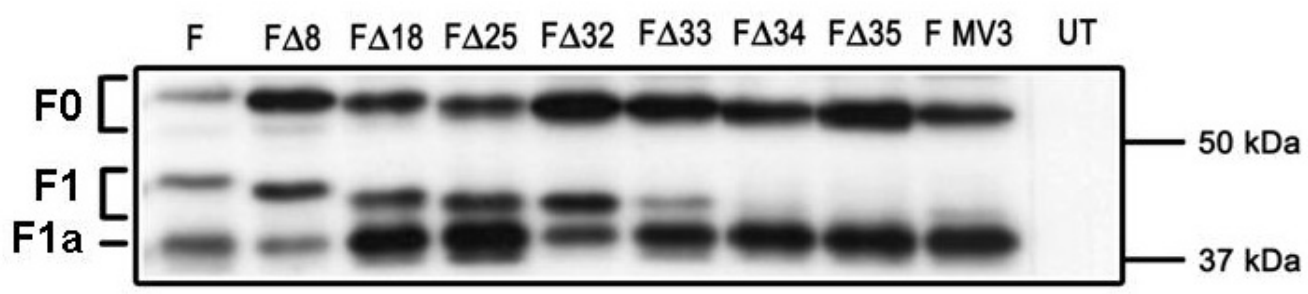

Figure 12: Scheme of the TPMV $F$ protein and sequences of the truncated cytoplasmic tails and Western blot analysis of different $F$ protein variants

TPMV $F$ is expressed as $F 0$ precursor protein and cleaved during activation, resulting in the fragments F1 and F2 (A). For pseudotyping, the cytoplasmic tail (CT) was truncated as indicated (B) or replaced by the truncated cytoplasmic tail of Measles virus $F$ protein ( $F M V 3)$. All proteins were expressed in $293 \mathrm{~T}$ cells, fractionated by SDS-10\% PAGE, and detected with a peptide antiserum against the ectodomain of the $\mathrm{F}$ protein $\left(\alpha \mathrm{F}_{\text {ecto, }} \mathrm{C}\right)$

TM: transmembrane domain; SP: signal peptide; FP: fusion peptide, UT: untransfected cells 
The production of the plasmid encoding the unmodified TPMV $F$ protein was already described (Springfeld et al., 2005). Corresponding to the TPMV H protein, the cytoplasmic tail of the $F$ protein was truncated at the carboxyterminus by $8,18,25$, $32,33,34$, and 35 amino acids, leaving only three residues on the shortest variant (Fig. 12, B). This was done by PCR using a reverse primer that introduced a stop codon and an Xbal cleavage side behind the respective amino acid in the plasmid PCG-TPMV-F at the 3 ' end of the protein coding sequence. The forward primer introduced a BamHI at the 5' end of the PCR product. Additionally, a protein was generated that has the same three-amino acid cytoplasmic tail (arginine - glycine arginine) as the recently described MV protein that was used successfully to pseudotype lentiviruses (Funke et al., 2008a). In Western blots, all F protein variants were expressed at a similar level and according to their predicted sizes (Fig. 12, C). The proteins were detected with a novel antibody generated against a peptide in the ectodomain of the $\mathrm{F}$ protein $\left(\mathrm{aF}_{\text {ecto }}\right)$. Surprisingly, with this antibody an additional fragment with a molecular mass of about $45 \mathrm{kDa}$ was detected, suggesting that the $\mathrm{F}$ protein is cleaved twice in addition to the removal of the signal peptide, resulting in the fragments $F 1 a, F 1 b$ and F2. However, in the proteins with the shortest cytoplasmic tails ( $F \Delta 34, F \Delta 35$ and $F M V 3$ ), the $F 1$ fragment was barely detectable, suggesting that in these proteins the $\mathrm{F} 1 \mathrm{a} / \mathrm{F} 1 \mathrm{~b}$ cleavage was more efficient than in the unmodified protein. The F1a/F1b cleavage is further characterized in section 6.2.

\subsubsection{Surface expression of the modified TPMV H proteins}

For further investigation of the modified TPMV glycoproteins the level of protein expression of the $\mathrm{H}$ protein on the cell surface was tested. Since surface expression is a critical step in vector formation (Sakalian and Hunter, 1998; Sandrin et al., 2004) it is important to investigate if the proteins are processed correctly after their synthesis and displayed on the cell surface. The $\mathrm{H}$ protein was heavily modified by fusing the scAb to the carboxyterminus and truncating the $\mathrm{CT}$ and might therefore not be properly processed. For this purpose, 293T cells were transfected with the respective TPMV $\mathrm{H}$-plasmids and 48 hours after transfection the cells were harvested and incubated with the primary antibody $\mathrm{H}_{\text {ecto }}$ and the FITC-labeled secondary antibody goat- $\alpha$-rabbit-lgG. Surface expression was then analyzed by fluorescence-activated cell-sorting (FACS) and the geometric mean levels of 
fluorescence intensity, representing the average amount of surface-expressed $\mathrm{H}$ protein, were compared. Surface expression levels were normalized to that obtained after expression of the unmodified TPMV H protein (100\%).

Surface expression of almost all modified protein variants was not relevantly inhibited by the aCD20-scAb (80 - $100 \%$ ) (Fig. 13), in contrast, for two variants it was slightly increased (112.3\% for $\mathrm{H} \triangle 40 \mathrm{aCD} 20$ and $116.9 \%$ for $\mathrm{H} \Delta 84 \alpha \mathrm{CD} 20)$. Only for the variants $\mathrm{H} \triangle 70 \mathrm{aCD} 20$ and $\mathrm{H} \triangle 60 \mathrm{aCD} 20$ the expression level is significantly reduced (56.2 \% and $66.9 \%$, respectively).
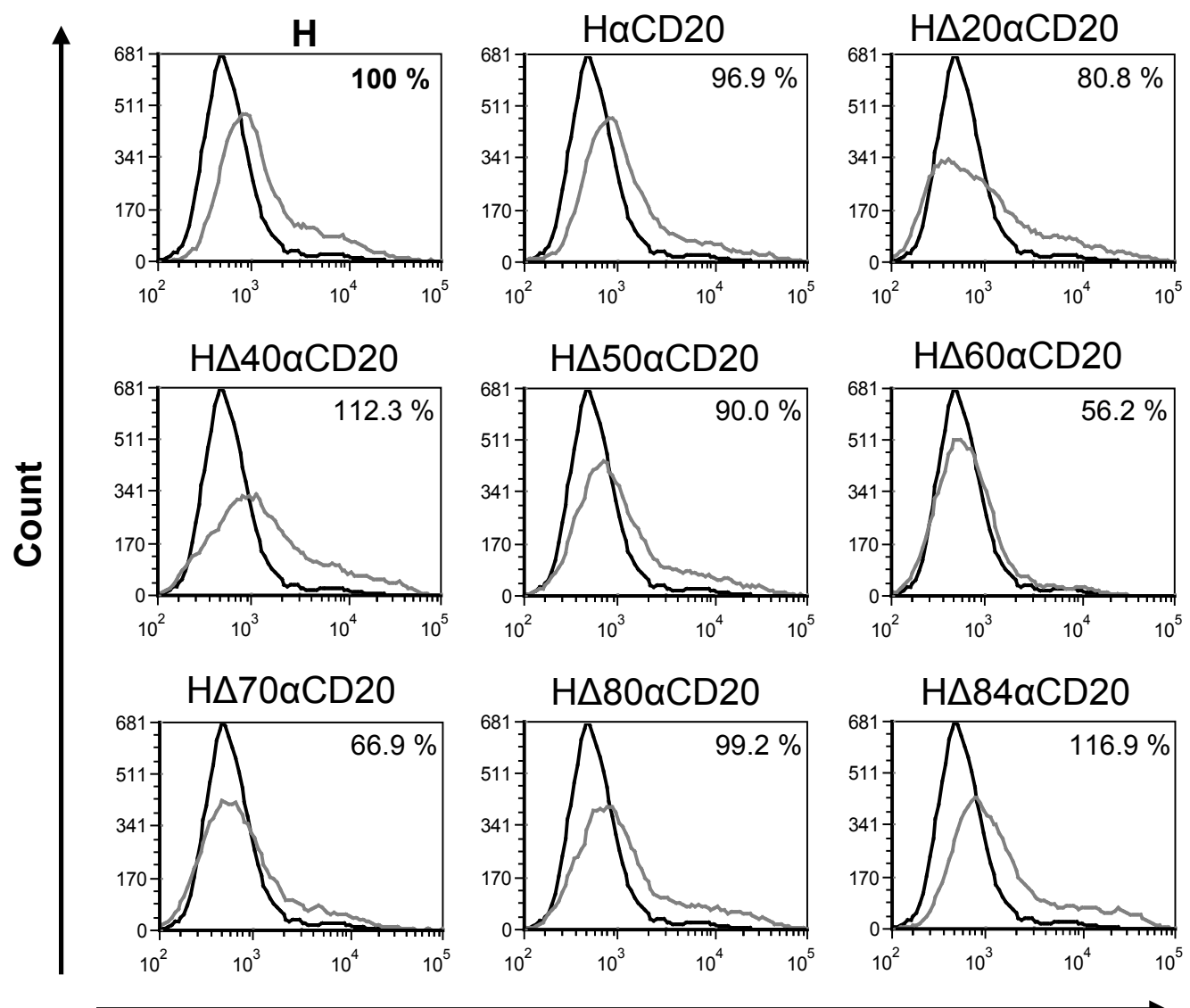

\section{FITC}

Figure 13: Cell surface expression levels of the different TPMV H protein variants

293T cells were mock-transfected (black line) or transfected with the different TPMV H expression plasmids (grey line), respectively. After 48 hours, mock- and $\mathrm{H}$-transfected cells were stained for surface expression using the antibody $\alpha \mathrm{H}_{\text {ecto }}$ and the fluorescence-labeled secondary antibody $\alpha-$ rabbit-FITC and analyzed by FACS. The geometric mean levels of fluorescence intensity, representing the average amount of surface-expressed $\mathrm{H}$ variants, were normalized to that obtained after expression of the unmodified TPMV H protein (100\%, upper left).

For the TPMV F variants, no antibody is available that allows FACS analysis of surface expression. 


\subsubsection{Functional analysis of the truncated TPMV protein variants}

Since surface expression of the modified TPMV H proteins is not the only critical step, next the functionality of the truncated protein variants was investigated. To test, if the TPMV $\mathrm{H}$ proteins with shortened cytoplasmic tail were still able to fulfill their fusion helper function and if the truncated TPMV F proteins still mediate membrane fusion, HT1080 cells that stable express CD20 were co-transfected with the wildtype $\mathrm{F}$ protein together with all HaCD20 protein variants and vice versa. Are the proteins functional, the cells fuse and form syncytia. For transfection control and for a better visualization of syncytia formation, a plasmid encoding GFP was transfected together with the TPMV-encoding plasmids. Fourty-eight hours after transfection, the cells were monitored for the formation of syncytia.

Unexpectedly, $F$ proteins with a cytoplasmic tail shorter than 5 amino acids ( $F \Delta 34$, $\mathrm{F} \triangle 35$ and $\mathrm{F} \mathrm{MV3}$ ) together with $\mathrm{HaCD} 20$ did not support fusion at all, whereas all proteins with longer CTs supported fusion similar to the wildtype F protein (Fig. 14 A). This indicates that at least 5 amino acids of the cytoplasmic tail of the $F$ protein are necessary to mediate membrane fusion. These results correlate with the lack of the fusion-triggering F1 fragment in these proteins in Western blots (Fig. $12 \mathrm{C}$ ). As a control, cells were transfected with a GFP-encoding plasmid only and as expected, no syncytia formation was observed. 


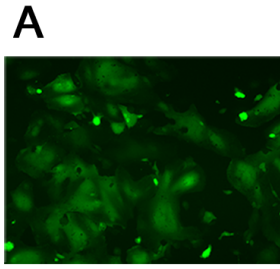

$\mathrm{F}(+++)$

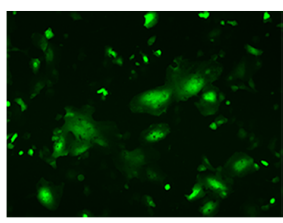

$\mathrm{F} \Delta 33(++)$

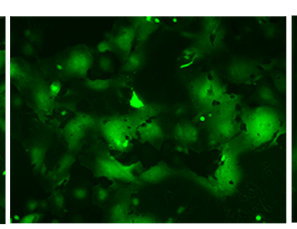

$\mathrm{F} \Delta 8(+++)$

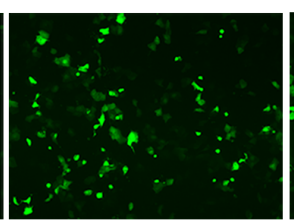

$\mathrm{F} \Delta 34(-)$

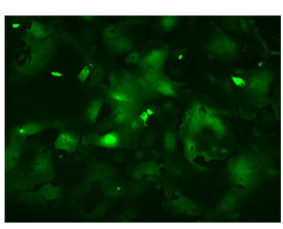

$\mathrm{F} \Delta 18(+++)$

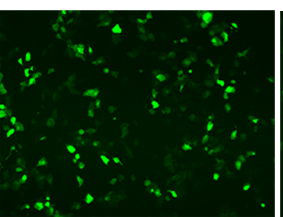

$\mathrm{F} \Delta 35(-)$

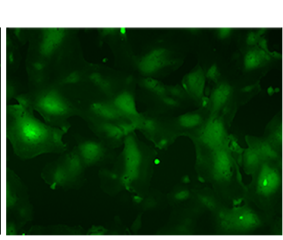

$\mathrm{F} \Delta 25(+++)$

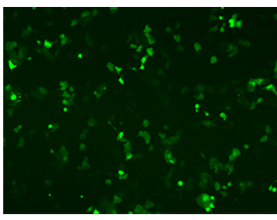

F MV3 (-)

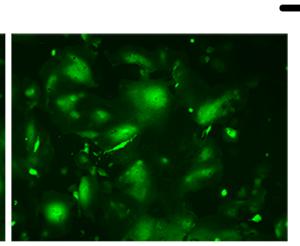

$\mathrm{F} \Delta 32(+++)$

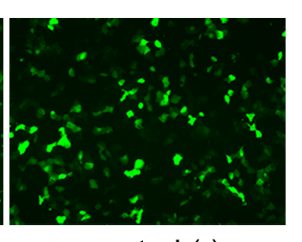

control (-) $\mathrm{H} \alpha \mathrm{CD} 20$

B

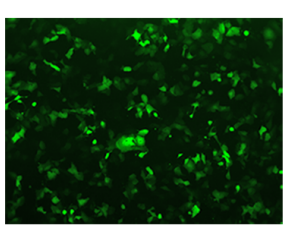

$\mathrm{H}(-)$

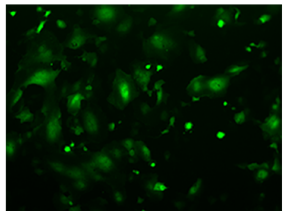

$\mathrm{H} \Delta 60 \alpha \mathrm{CD} 20(++)$

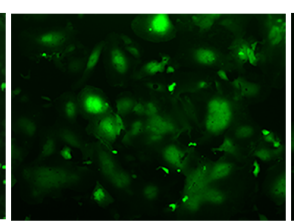

$\mathrm{H} \alpha \mathrm{CD} 20(+++)$
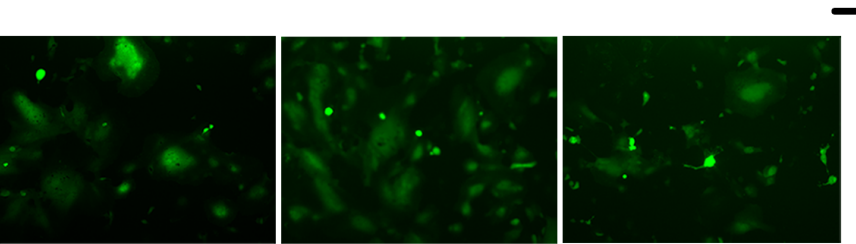

$\mathrm{H} \Delta 20 \alpha \mathrm{CD} 20(+++) \mathrm{H} \Delta 40 \alpha \mathrm{CD} 20(+++) \mathrm{H} \Delta 50 \alpha \mathrm{CD} 20(+++)$

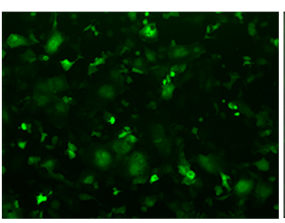

$\mathrm{H} \triangle 80 \alpha \mathrm{CD} 20(+)$

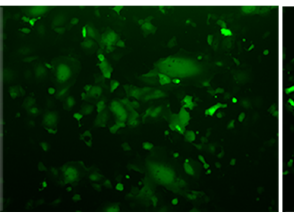

$\mathrm{H} \Delta 84 \alpha C D 20(++)$

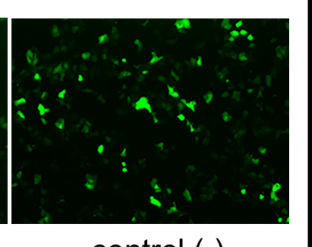

control (-)

Figure 14: Functional analysis of the modified TPMV glycoproteins in syncytia assays

HT1080-CD20 cells were transfected with plasmids encoding the modified proteins and a plasmid encoding eGFP to test their ability to induce syncytia formation. The untruncated TPMV HaCD20 together with all $F$ protein variants $(A)$ and unmodified TPMV $F$ with all TPMV $H$ variants $(B)$ were tested. Fourty-eight hours after transfection, GFP expression was analyzed by fluorescence microscopy (100x magnification). Control: cells transfected with GFP-encoding plasmid only; GFP: green fluorescent protein; $(+)$ and $(-)$ indicates the size of syncytia formation

In contrast to the results with the $F$ proteins, there was no similar cut-off with a complete loss of function observed when the truncated $\mathrm{H}$ proteins were tested together with TPMV F (Fig. $14 \mathrm{~B}$ ). However, the degree of fusion was slightly reduced in proteins with larger truncation at the cytoplasmatic tail when compared to the wildtype. As expected, the untruncated TPMV $\mathrm{H}$ protein without the $\operatorname{scAb}(\mathrm{H})$ does not form syncytia because of the lack of a receptor for TPMV on HT1080 cells. 


\subsection{Identification of a novel TPMV fusion protein fragment}

As already mentioned, the TPMV F protein is expressed as a F0 precursor protein and cleaved during activation. To characterize the TPMV F protein mutants with truncated modified cytoplasmic tail for pseudotyping lentiviral vectors, a novel antibody against a peptide in the ectodomain of the $F$ protein was generated. Surprisingly, with this antibody a novel fragment with a molecular mass of about 48 $\mathrm{kDa}$ was detected, suggesting that the $\mathrm{F}$ protein is cleaved twice in addition to the removal of the signal peptide, resulting in the fragments F1a, F1b and F2 (Fig. $12 \mathrm{~A}$, C). In this part of the result section, the additional cleavage of the TPMV F protein is further described.

\subsubsection{Further characterization of the TPMV F protein with the novel antibody $F_{\text {ecto }}$}

The TPMV F gene is located between the gene for the M protein and the attachment (H) protein, showing the typical paramyxovirus gene order (Fig. 15, A). The 553 amino acid protein (61 kDa) has three hydrophobic regions, the signal peptide (SP), the fusion peptide (FP), and the transmembrane domain (TM) (as predicted by TMHMM, http://www.cbs.dtu.dk/services/TMHMM) (Fig. 15, B). In the ectodomain there are four possible glycosylation sites, three on the F2 fragment (11 kDa) and one on the $\mathrm{F} 1$ fragment (50 kDa), which are all glycosylated (Fig. 15, B, triangles) (Springfeld et al., 2005). The novel antibody was generated against the stalk region

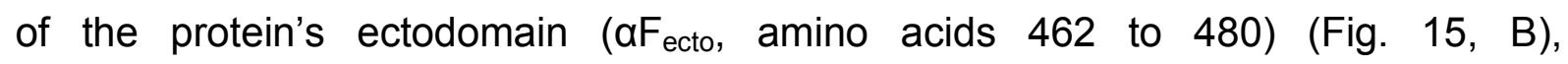
corresponding to the peptide ELEMDKTQKALDRSNKIL (Fig.15, C, written in bold) which is located in the heptad repeat region B (HRB) (von Messling et al., 2004). Since a novel fragment around $45 \mathrm{kDa}$ can be detected with $\alpha \mathrm{F}_{\text {ecto }}$, an additional $\mathrm{F}$ protein cleavage has to occur downstream of the $\alpha F_{\text {ecto }}$ binding peptide. Alignment of this part of the ectodomain and the transmembrane region of selected paramyxoviruses reveals a certain degree of sequence conservation with a characteristic pattern of hydrophobic amino acids (Fig. 15, C). 


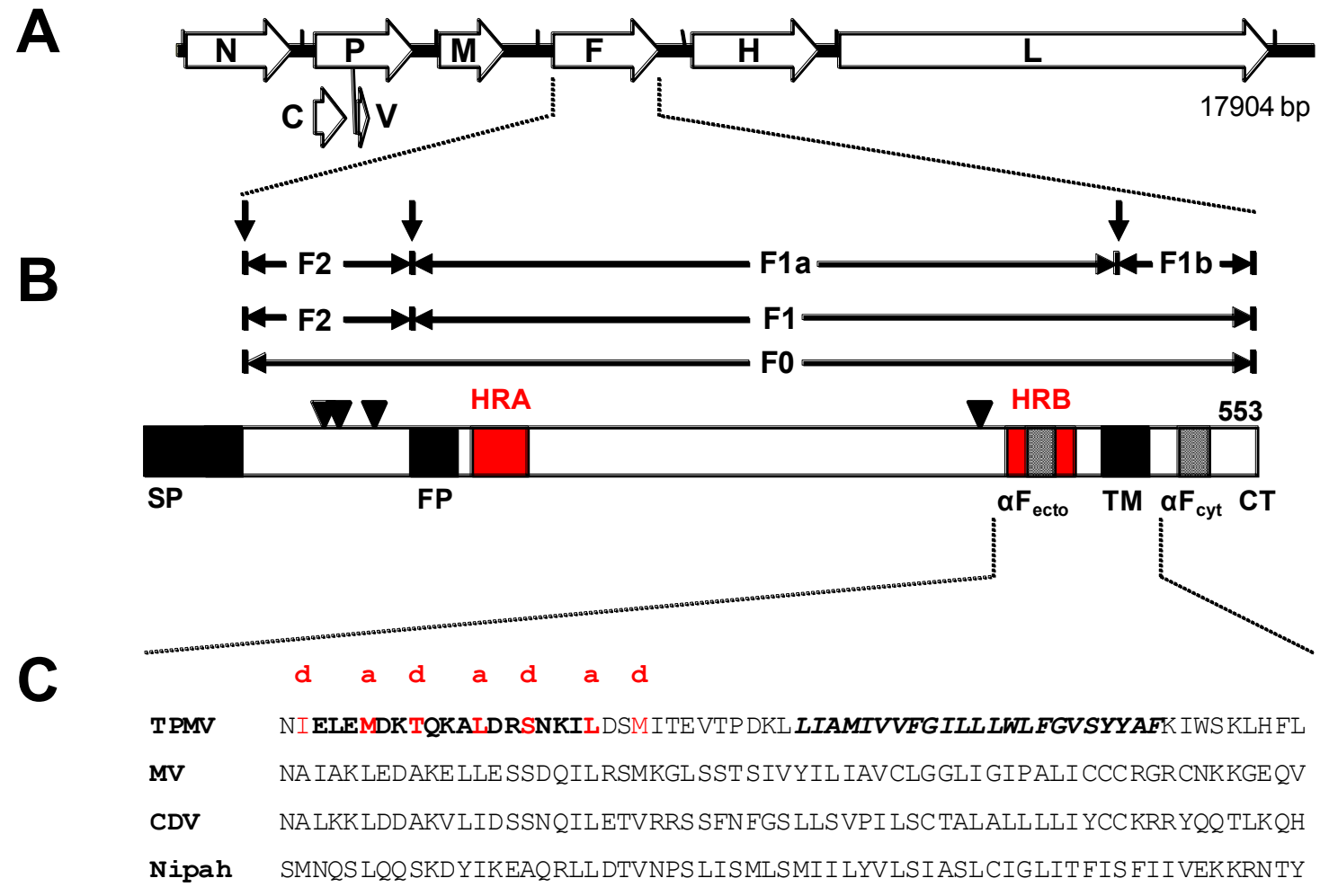

Figure 15: Genome organization of TPMV and structure and sequence of the F protein

The map of the TPMV genome shows typical paramyxovirus gene order $(A)$. In the linear drawing of the TPMV $F$ protein (B), the black triangles ( $\mathbf{\nabla})$ indicate the glycosylation sites and the arrows the cleavage sites of the F0 precursor protein. Cleavage activation results in the fragments F1a, F1b and F2. The grey-colored boxes mark the peptide binding side of the antibodies $\mathrm{\alpha F}_{\text {ecto }}$ and $\mathrm{\alpha F}_{\text {cyt }}$. The redcolored boxes indicate the positions of the heptad repeat regions HRA and HRB. Alignment of the $\mathrm{F} 1 \mathrm{a} / \mathrm{F} 1 \mathrm{~b}$ junction, the $\alpha \mathrm{F}_{\text {ecto }}$ binding site (in boldface) and the predicted transmembrane domain (in boldface and italic) of TPMV, MV, CDV and Nipah virus shows only low similarities (C). Identical amino acids are indicated by an asterisk, highly conserved amino acids are indicated by a colon, and well conserved amino acids are indicated by a period. The hydrophobic residues of the first and fourth (a and d) positions of heptad repeat region B (HRB) are in red color. HRA: heptad repeat region A; SP: signal peptide; FP: fusion peptide; TM: transmembrane domain; CT: cytoplasmic tail; MV: measles virus; CDV: canine distemper virus

\subsubsection{The novel F1a fragment is transported along the secretory pathway and cleaved at the carboxy-terminus}

For further characterization of the novel TPMV F1a fragment, the glycosylation pattern was investigated. For this purpose, 293T cells were transfected with a plasmid encoding TPMV $F$ and the protein content of the lysate was determined by Bradford assay. Subsequently, the lysate was incubated either with the deglycosylation enzyme PNGase F or Endoglycosidase H (Endo H), applied to SDSpolyacrylamide-gelelectrophoresis and analyzed by Western Blot using the novel 
$\mathrm{F}_{\text {ecto }}$ antibody and an antibody against the $\mathrm{C}$ terminus of the TPMV $\mathrm{F}$ protein $\left(\mathrm{aF}_{\text {cyt, }}\right.$, Fig. 15, B).

In lysate analyzed by Western Blot using the $\mathrm{F}_{\text {ecto }}$ antibody the novel fragment F1a with a molecular mass of about $45 \mathrm{kDA}$ was detected, but not with an antibody against the cytoplasmic tail (Fig. 16, A) (Springfeld et al., 2005). The lysate was incubated with PNGase $F$, a deglycosylation enzyme that catalyzes the complete removal of $\mathrm{N}$-linked oligosaccharide chains from glycoproteins. Digestion with PNGase $F$ resulted in fragments with smaller molecular weight, confirming that the glycosylation residues were removed. The shift of the F0 fragment was larger compared to the F1 and F1a fragment, accordingly to the removal of four glycosylation residues on the F0 and only one on the F1 and F1a fragment. To investigate whether the new F1a fragment is expressed on the cell surface, the lysate was incubated with Endo $\mathrm{H}$. This enzyme is used to monitor posttranslational modifications in the Golgi apparatus. It removes only high mannose and some hybrid oligosaccharides from $\mathrm{N}$-linked glycoproteins which are added before the protein passes the medial Golgi region, but it does not remove complex oligosaccharides. Proteins, that are resistant to Endo $\mathrm{H}$, are correctly processed through the Golgi, containing complex oligosaccharides added in the trans-Golgi and rapidly transported to the cell surface. Endo $\mathrm{H}$-sensitive proteins have not been processed beyond the medial Golgi region. As expected, the precursor protein F0 was mostly sensitive towards Endo H (Fig. $16 \mathrm{~A}$ ) resulting in a shift of the signal, respectively. The F1 and F1a fragment were almost completely resistant towards the enzyme. These data demonstrate that the F0 fragment was mainly located intracellular whereas the F1 and also the F1a fragment were transported to the cell surface. Two additional bands around 60 and $70 \mathrm{kDa}$ were detectable in lysate of TPMV F incubated with Endo $\mathrm{H}$. Since these signals were also detectable in lysate of untransfected cells together with Endo $\mathrm{H}$, they were probably due to unspecific binding of the $\mathrm{aF}_{\text {cyt }}$ antibody to the enzyme. 
A

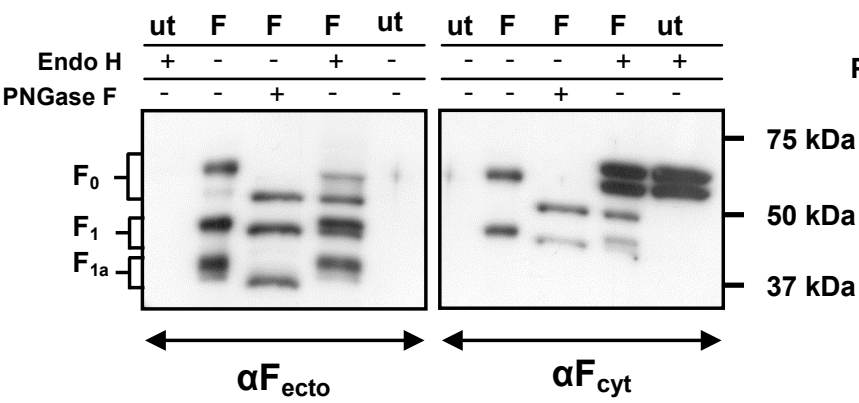

B

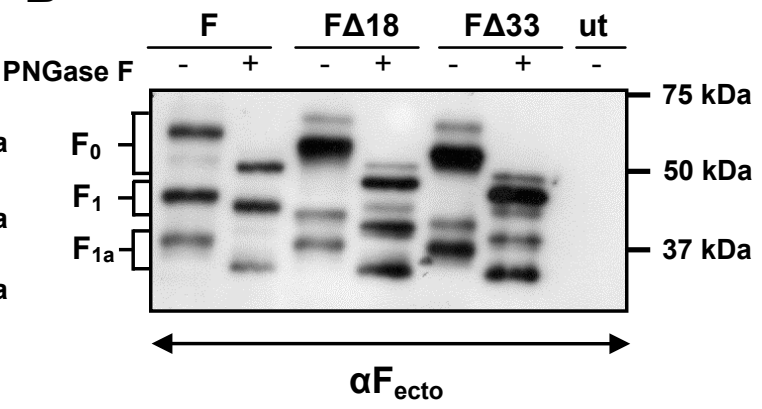

Figure 16: Deglycosylation of TPMV $F$ variants and Western Blot analysis using $\alpha F_{\text {ecto }}$ and $\alpha F_{\text {cyt }}$ The TPMV $F$ protein variants were expressed in 293T cells, the lysate incubated with Endo $\mathrm{H}$ or PNGase $\mathrm{F}$ and fractioned by SDS-10\% PAGE followed by detection with $\alpha \mathrm{F}_{\text {ecto }}$ and $\alpha \mathrm{F}_{\text {cyt }}$.

Deglycosylation with PNGase $F$ resulted in a shift of F0, F1 and F1a fragment whereas the F1a fragment is resistant to Endo $\mathrm{H}$ and could not be detected with $\mathrm{aF}_{\text {cyt }}(A)$. After PNGase $F$ incubation of $\mathrm{F}$ variants truncated at their cytoplasmic tails, same deglycosylation pattern of $\mathrm{F} 1 \mathrm{a}$ was detected as for the unmodified $F$ protein (B).The positions of F0, F1 and F1a are indicated on the left. ut: untransfected cells

Next, it was investigated, whether the F1a fragment was truncated at the $\mathrm{N}$ - or Cterminus with regard to the F1 fragment. Two selected proteins with truncated cytoplasmic tail (F $\Delta 18, F \Delta 33$ ) were compared to the native TPMV F protein. 293T cells were transfected with the respective expression plasmids and the protein content of the lysate was determined by Bradford assay. Afterwards, the lysate was digested with PNGase F, applied to SDS-polyacrylamide-gelelectrophoresis and analyzed by Western Blot using the novel $F_{\text {ecto }}$ antibody.

The F0 and the F1 fragment of the truncated proteins migrated faster according to their molecular weight, while the F1a fragment stayed at the same height (Fig. 16, B) compared to the unmodified TPMV F protein. This observation demonstrates that the F1 fragments were cleaved at the carboxy-terminus, since the molecular weight of the F1a fragment was not affected by truncation of the cytoplasmic tail. Hence, the cleavage site was estimated to be located closely proximal to the transmembrane domain of the protein (amino acids 493 to 515, Fig. 15, C). Since $\alpha \mathrm{F}_{\text {ecto }}$ is directed against amino acids 462 to 480 , cleavage has to occur downstream of these amino acids. It is noteworthy, that a strong F1 signal is detectable in all lysates, demonstrating that the second cleavage occurred only in a fraction of the $F$ protein.

To demonstrate that the second cleavage occurs also in cells naturally infected by the Tupaia paramyxovirus, Tupaia baby fibroblasts (TBFs) were transfected with expression plasmids for TPMV F, F $\Delta 18$ and F $\Delta 33$. The lysate was harvested 48 hours after transfection and protein content determined by Bradford assay. 
Subsequently, the lysate was applied to SDS-polyacrylamide-gelelectrophoresis and Western Blot analysis was performed using $F_{\text {ecto }}$ antibody.

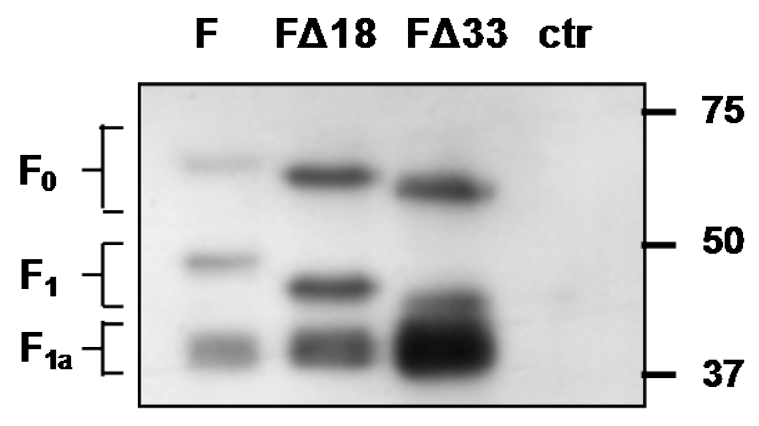

Figure 17: F0, F1a and F1a fragment detection of TPMV F variants expressed in TBF cells

TBF cells were transfected with TPMV F protein variants and the proteins separated by SDS- $10 \%$ PAGE followed by Western Blot analysis using $\mathrm{\alpha F}_{\text {ecto }}$. F0, $\mathrm{F} 1$ and $\mathrm{F} 1 \mathrm{a}$ were detected after expression in TBF cells. The positions of F0, F1 and F1a are indicated on the left. ut: untransfected cells

All three TPMV F protein fragments (F0, F1, F1a) were detected in cell lysate of Tupaia baby fibroblasts after transient transfection with different TPMV F expression plasmids (Fig. 17), confirming, that the second F1a/F1b cleavage occurs also in cells naturally infected by TPMV. Since TBFs are primary cells and difficult to transfect, all further experiments were performed in 293T cells if not indicated different.

\subsubsection{The TPMV $F$ protein ectodomain is shed into the supernatant of cells}

Next, it was tested whether the F1a fragment was shed into the supernatant of cells expressing TPMV $F$ and the modified variants $F \Delta 18$ and $F \Delta 33$. For this purpose, 293T cells were transiently transfected with plasmids expressing TPMV F, F $\Delta 18$ and $\mathrm{F} \Delta 33$. Fourty-eight hours after transfection, the supernatant of the cells and the lysate was harvested. Protein content of the lysate was determined by Bradford assay and applied to SDS-polyacrylamide-gelelectrophoresis. Of the supernatant, the highest possible volume was applied with subsequent Western Blot analysis using $F_{\text {ecto }}$ antibody.

Indeed, the F1a fragment was detected in all supernatants of 293T cells transfected with the different plasmids expressing TPMV F protein variants (Fig. 18) and almost no amounts of FO and F1, suggesting, that F1a was secreted and released in the supernatant of the cells. Since it was predicted that the membrane anchor is cleaved of, the presence of the F1a fragment was expected. 


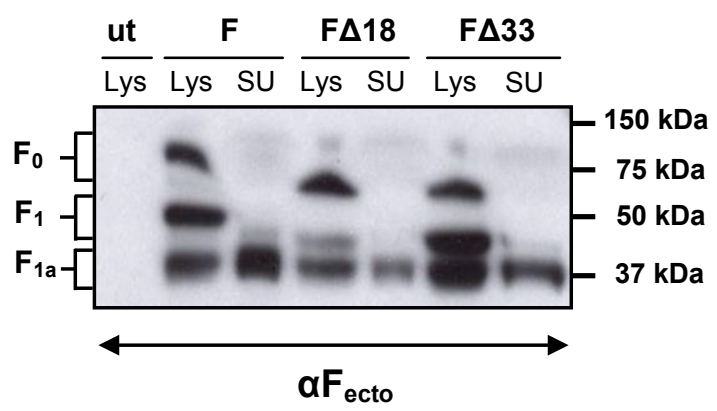

Figure 18: TPMV F1a fragment is shed into the supernatant of cells

Transfection of 293T cells with TPMV F protein variants. The lysate and supernatant were harvested and proteins were separated by SDS-10\% PAGE followed by Western Blot analysis using $\mathrm{aF}_{\text {ecto. }}$. The positions of F0, F1 and F1a are indicated on the left. ut: untransfected cells; Lys: lysate; SU: supernatant; inf. Infected TBF cells

\subsubsection{The TPMV F1a fragment is present in TPMV virions}

Next, it was investigated if the F1a fragment is also present in TPMV virions. Although the F1a fragment has lost its membrane anchor, it could possibly be present in $\mathrm{F}$ protein trimeric complexes as described previously for measles virus (von Messling et al., 2004). For this purpose, TBFs were infected with TPMV and after 5-6 days, the supernatant (SU) and lysate (Lys) of infected and non-infected cells were harvested and analyzed as described before. To exclude detection of F0, $\mathrm{F} 1$ and $\mathrm{F} 1 \mathrm{a}$ in the supernatant due to cell lysis after syncytia formation or shedding, the TPMV-virion-containing supernatant was concentrated and purified by ultra centrifugation (UC) over a $20 \%$ sucrose cushion. Only $2.5 \mu \mathrm{g}$ of total protein content of concentrated virions was analyzed compared to lysate, whereat a total protein concentration of $10 \mu \mathrm{g}$ was applied.

Indeed, the F1a fragment was clearly detectable in high amounts in purified TPMV virions (Fig. 19). Surprisingly, an additional fragment around $47 \mathrm{kDa}$ was detected in virions, which was not present in lysate of infected TBF cells, suggesting that $F$ protein processing is even more complex. As expected, no F0 fragment was detectable in TPMV virions. 


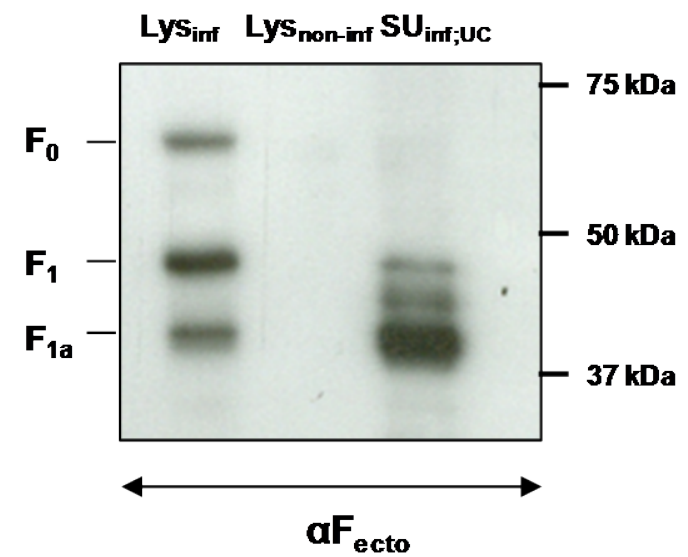

Figure 19: The F1a fragment is present in TPMV virions

Tupaia baby fibroblasts (TBF cells) were infected with TPMV and lysate and supernatant were harvested. TPMV-virion-containing supernatant was concentrated and purified by ultra centrifugation (UC) over a $20 \%$ sucrose cushion. Proteins were separated by SDS-10\% PAGE followed by Western Blot analysis using $\alpha F_{\text {ecto }}$. The positions of F0, F1 and F1a are indicated on the left. Lys inf: Iysate of

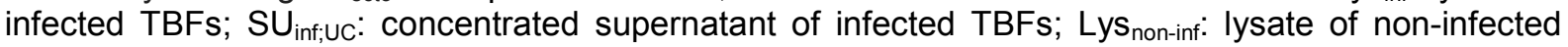
TBFs

\subsection{Characterization of the newly identified cleavage site}

Having demonstrated that the novel F1a fragment is not only secreted but cleaved at the carboxy-terminus, now the cleavage site between the F1a and F1b-fragment was characterized.

\subsubsection{Mapping of the F1a-F1b cleavage site}

To determine the cleavage site between the F1a and F1b-fragment, eleven cterminally truncated proteins were generated with a Flag-tag added at positions 483, 491, 492, 495, 498, 501, 505, 507, 510, 513 and 516 (Fig. 20, modified from (von Messling et al., 2004)) (Fig. 21, A) and detected with an aFlag-antibody and the

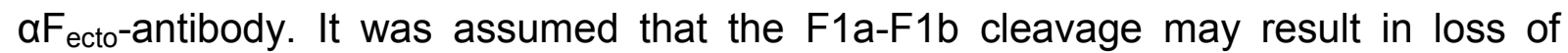
protein detection, as the aFlag-antibody does not recognize the cleaved protein anymore. 


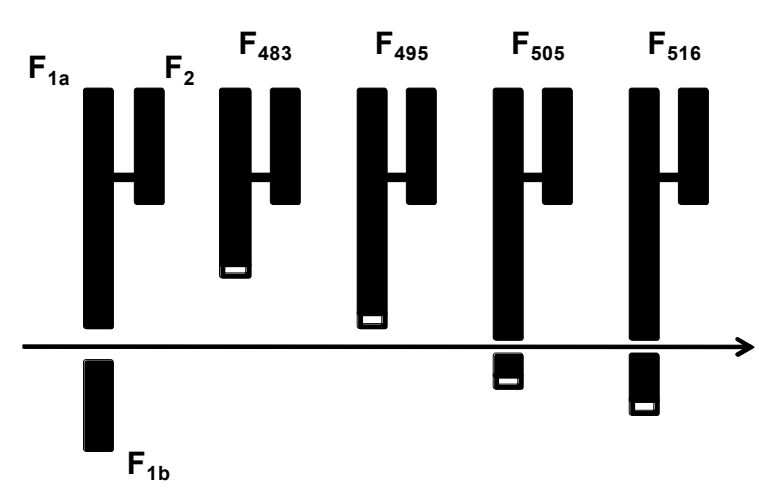

Figure 20: Schematic drawing of truncated Flag-tagged TPMV F variants

The TPMV F protein was truncated at indicated amino acid positions and a Flag-tag (white box) was fused at the carboxy-terminus, resulting in shorter F1a and F1b fragments compared to the native $F$ protein (left). The arrow indicates the expected position of F1a/F1b cleavage. The line connecting F1a and $\mathrm{F} 2$ fragments symbolizes the disulfide bond. Only four representative Flag variants are shown here, $\mathrm{F}_{438}, \mathrm{~F}_{495}, \mathrm{~F}_{505}$ and $\mathrm{F}_{516}$.

The different truncated TPMV F-Flag-variants were generated by PCR using primers that introduced a Flag-tag at defined amino acid positions, followed by a stop codon. Afterwards, 293T cells were transfected with the respective expression plasmids and 48 hours later, the lysate was harvested and protein content determined by Bradford assay. The lysate was then applied to SDS-polyacrylamide-gelelectrophoresis and analyzed by Western Blot using the antibodies $\alpha F$ lag and $\alpha F_{\text {ecto }}$.

All F0 fragments were detected using $\alpha F_{\text {ecto }}(F i g .21, B)$. However, when the same cell lysates were tested in Western Blots with aFlag antibody, TPMV F Flag variants longer than 498 amino acids were not detected anymore, suggesting that cleavage of F1a/F1B occurred between amino acid position 498 and 501. Surprisingly, there was no F1 fragment detectable with aFlag-antibody. Regarding Western Blot analysis with $\mathrm{aF}_{\text {ecto, }}$, there was the $\mathrm{F} 0$ fragment and a strong signal around $40 \mathrm{kDa}$, suggesting that this was shortened F1 together with $\mathrm{F} 1 \mathrm{a}$. In $\mathrm{F}_{\mathrm{Flag}}$ variants longer than 498 amino acids, the F1 together with F1a signal was strongly decreased. Currently, these observations cannot be explained and further investigations are necessary. Nevertheless, cleavage site of F1a/F1b was mapped between amino acids 498 and 501. To map the cleavage site in detail, two further protein variants were generated with a flag-tag after amino acid position 499 and 500 and analyzed by Western Blot as described before (Fig. 21, C). The variant TPMV $F_{500}$ was not detected anymore by aFlag-antibody, demonstrating that cleavage occurred exactly between amino acid 499 and 500. Surprisingly, this position was located in the predicted transmembrane domain. Again, no F1 fragments were visible using aFlag antibody. In contrast, F1 was detected when the Flag-tag was added after amino acid position 
533, which is located in the cytoplasmic tail, or at the C-terminal end of the protein (amino acid position 553). However, some unexpected results were obtained that cannot be explained and might be due to the heavy modifications of the protein.

A

NIELEMDKTQKALDRSNKILDSMITEVTPDKLLIAMIVVFGILLLWLFGVSYYAFK IWS . .

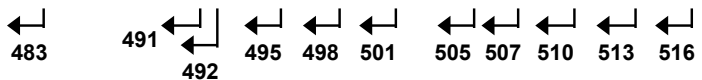

B
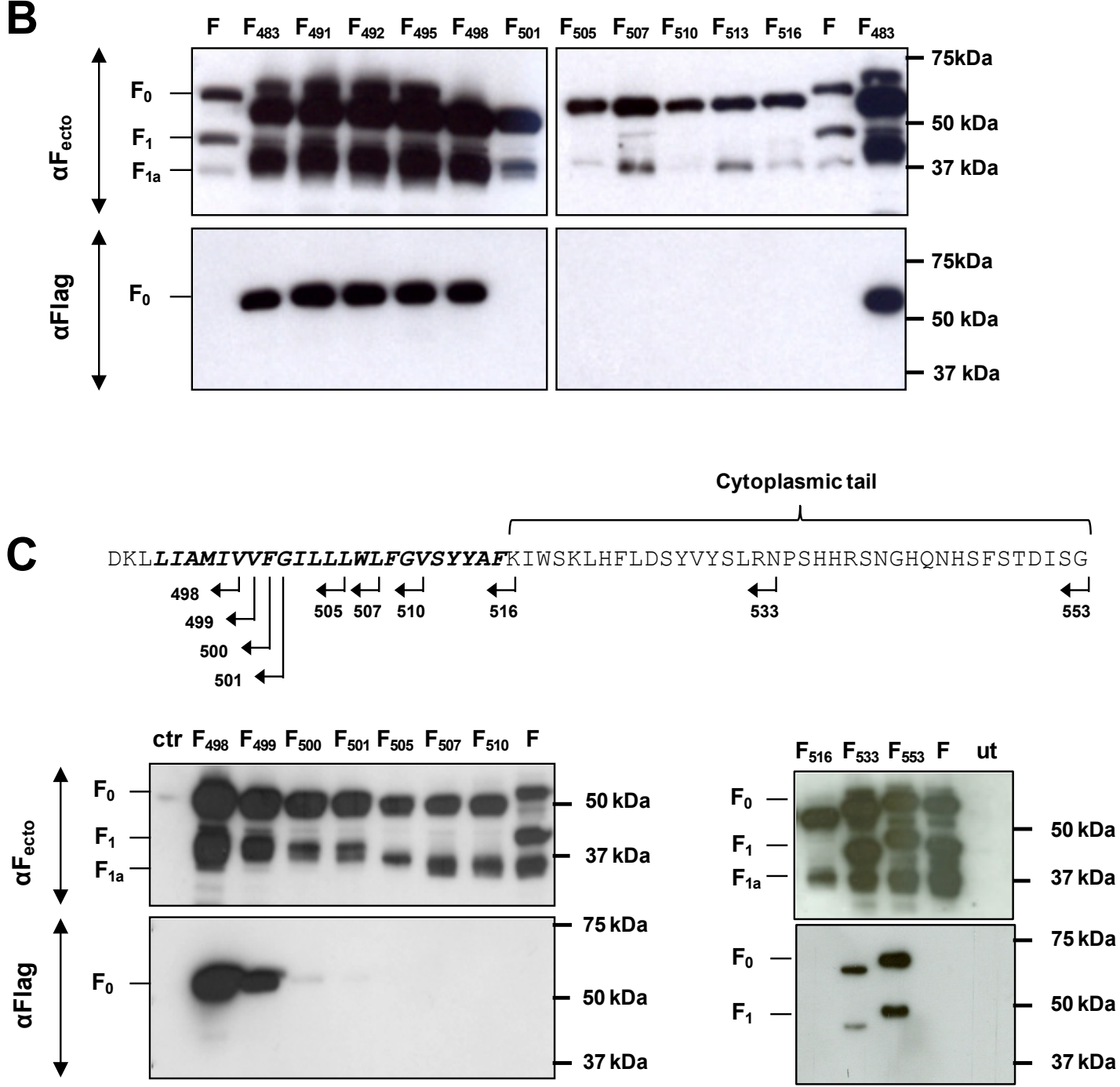

Figure 21: Mapping of F1a/F1b cleavage site by Western Blot analysis of TPMV F Flag-variants Scheme of TPMV $F$ transmembrane region $(A)$ and mapping of the $F 1 \mathrm{a} / F 1 \mathrm{~b}$ cleavage site $(B$ and $C)$. (A) The TPMV F protein was truncated at the indicated amino acid (aa) positions (number and arrow) and a Flag-tag was added at the truncation site. The binding peptide of the antibody aFecto is in bold, the predicted transmenbrane domain in bold and italics. (B) Western Blot analysis of truncated Flagtagged F protein variants. 293T cells were transfected with TPMV F Flag-tagged variants and the lysate was harvested. Proteins were separated by SDS-10\% PAGE followed by Western Blot analysis using the antibodies $\alpha \mathrm{F}_{\text {ecto }}$ and aFlag. (C) Detailed mapping of the cleavage site. A Flag-tag was added at single aa positions between aa 498, 501 and aa 533, 553 of TPMV F. Western Blot analysis was performed as described before. The positions of F0, F1 and F1a are indicated on the left. ut: untransfected cells 


\subsubsection{Mutation of the F1a-F1b cleavage site}

After identification of the exact amino acid positions of the $\mathrm{F} 1 \mathrm{a} / \mathrm{F} 1 \mathrm{~b}$ cleavage site, it was investigated whether cleavage was inhibited by mutation of the amino acids, respectively. For this purpose, two different cleavage mutants were generated by mutagenesis PCR replacing the three amino acids at position $498-500\left(\mathrm{~F}_{\mathrm{Ala} 1}\right)$ or at position 499-501 $\left(\mathrm{F}_{\mathrm{Ala} 2}\right)$ by alanin (Fig. 22, A). 293T cells were transfected with the respective expression plasmids and 48 hours later, the lysate was harvested and protein content determined by Bradford assay. The lysate was then applied to SDSpolyacrylamide-gelelectrophoresis and analyzed by Western Blot using $\mathrm{\alpha F}_{\text {ecto- }}$ antibody.

A

Cleavage site

. . NIELEMDKTQKALDRSNKILDSMITEVTPDKLLIAMTVVFGILLLGLFGVSYYAFKIWS..

FAla1 .. NIELEMDKTQKALDRSNKILLDSMI TEVTPDKLLIAMIAAAGGILLLWLFGVSYYAFKIWS . .

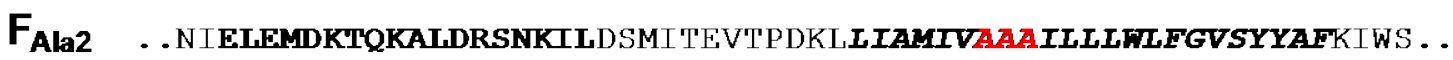

B

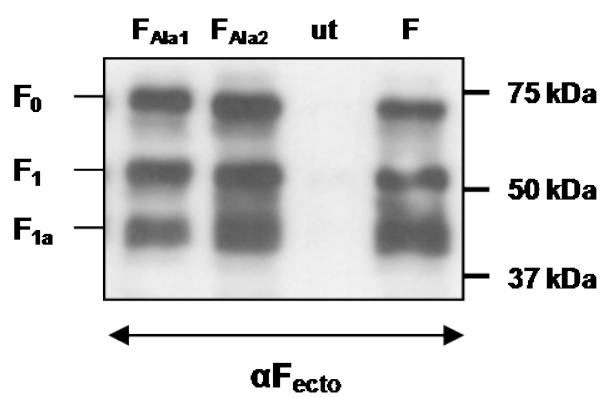

Figure 22: Mutation of the identified F1a-F1b cleavage site does not prevent F1a-F1b cleavage Amino acids surrounding the new identified $\mathrm{F} 1 \mathrm{a}-\mathrm{F} 1 \mathrm{~b}$ cleavage site were replaced by alanins resulting in the TPMV $F$ mutants $F_{\mathrm{Ala} 1}$ and $\mathrm{F}_{\mathrm{Ala} 2}(\mathrm{~A})$. The mutated amino acids are in red, the binding peptide of the antibody aFecto is in bold, the predicted transmenbrane domain in bold and italics. The identified cleavage site between valine and phenylalanine is indicated by an arrow. For Western Blot analysis of the F mutants (B), 293T cells were transfected with the respective expression plasmids and the lysate was harvested. Proteins were separated by SDS-10\% PAGE followed by Western Blot analysis using the antibody $\alpha \mathrm{F}_{\text {ecto. }}$. The positions of $\mathrm{F} 0, \mathrm{~F} 1$ and $\mathrm{F} 1 \mathrm{a}$ are indicated on the left. ut: untransfected cells

In both $F$ protein mutants, $F_{A l a 1}$ and $F_{A l a 2}$, the fragments $F 0, F 1$ and $F 1$ a were detected (Fig. 22, B). Compared to the unmodified TPMV protein, there was no difference in molecular size of the fragments. These data demonstrate that the F1/F2 and the F1a/F1b cleavage still occurred, assuming that the mutated amino acids are not necessary for $\mathrm{F}$ protein cleavage. 


\subsection{Characterization of the cleavage protease}

After mapping the $\mathrm{F} 1 \mathrm{a} / \mathrm{F} 1 \mathrm{~b}$ cleavage site, next step is to characterize the cleavage protease. As mentioned before, the $F$ protein is cleaved twice during activation. Although the first cleavage (F1/F2) is already described (Springfeld et al., 2005), the responsible protease is not yet identified. To get a first overview about the involved protease the commercially available protease inhibitor cocktail 'Complete' (Roche) was tested. One tablet of 'Complete' was dissolved in DMEM medium according to the manufactures instructions. 293T cells were transfected with pCG-TPMV-F expression plasmid and five hours after transfection, the medium was changed against medium supplemented with stepwise diluted concentrations of 'Complete'. After 48 hours, the lysate was harvested and protein content determined by Bradford assay. The lysate was then applied to SDS-polyacrylamide-gelelectrophoresis and analyzed by Western Blot using the $\mathrm{aF}_{\text {ecto-antibody. }}$

In the presence of high concentrated protease inhibitor, F0 was detected in very low amount, but no F1 and F1a fragment (Fig. 23). In lysates of transfected cells incubated with lower 'Complete' concentrations small amounts of F1 and F1a were presented in addition to the FO fragment.

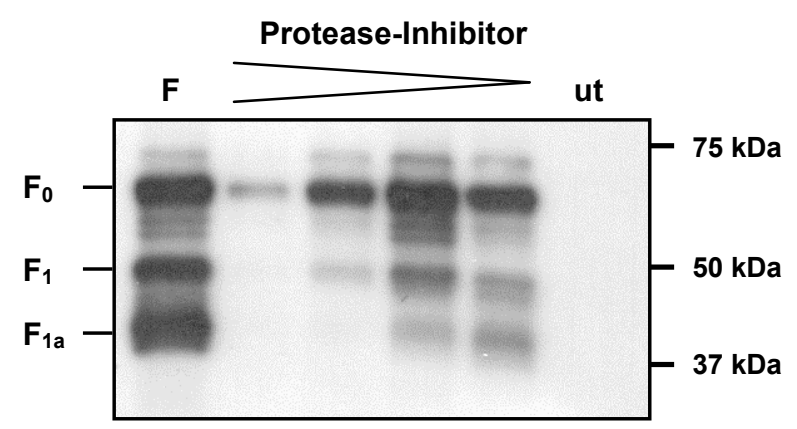

Figure 23: Protease inhibitor prevents F1-F2 and F1a-F1b cleavage

293T cells were transfected with TPMV F and five hours after transfection the medium was replaced by medium supplemented with 'Roche Complete' protease inhibitor cocktail in different concentrations. 48 hours later, the lysate was harvested and proteins were separated by SDS-10\% PAGE followed by

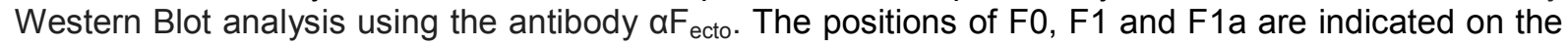
left. ut: untransfected cells

In conclusion, it has been shown that the amount of F1 and F1a increased with lower concentrations of 'Complete', demonstrating that both cleavages, F1/F2 and $\mathrm{F} 1 \mathrm{a} / \mathrm{F} 1 \mathrm{~b}$, can be inhibited by protease inhibitor added to the medium. Since the exact 
composition of this inhibitor is confidential, no conclusions about the protease can be drawn and further experiments have to be performed.

\subsubsection{Screening of different protease inhibitors}

Since it was shown that cleavage of the TPMV protein was prevented by incubation with the protease inhibitor cocktail 'Complete', the question raised if it is possible to identify the cleavage protease by analyzing different protease inhibitors separately. For this reason, 293T cells were transfected with TPMV F expression plasmid and treated with ten different protease inhibitors as described before. The inhibitors and their specificities are summarized in table 1 . They were added to the cells in concentrations suggested by the manufacturer (Roche, Mannheim).

\section{Table 1: Protease inhibitors and their specificities}

\begin{tabular}{|l|l|}
\hline Inhibitor & Specificity of Inhibitor \\
\hline $\begin{array}{l}\text { Antipain- } \\
\text { dihydrochloride }\end{array}$ & papain, trypsin is inhibited to a small extent \\
\hline Bestatin & $\begin{array}{l}\text { amino peptidase, including aminopeptidase B, leucine } \\
\text { aminopeptidase, tripeptide aminopeptidase }\end{array}$ \\
\hline Chymostatin & $\alpha-, \beta-, \gamma-$, -chymotrypsin \\
\hline E-64 & cysteine proteases \\
\hline Leupeptin & $\begin{array}{l}\text { serine and cysteine proteases such as plasmin, trypsin, } \\
\text { papain, cathepsin B }\end{array}$ \\
\hline Pepstatin & $\begin{array}{l}\text { aspartate proteases like pepsin, renin, cathepsin D, } \\
\text { chymosin }\end{array}$ \\
\hline Phosphoramidon & $\begin{array}{l}\text { metallo endopeptidases, specifically thermolysine, } \\
\text { collagenase, metallo endoproteinases }\end{array}$ \\
\hline Pefabloc SC & $\begin{array}{l}\text { serine proteases, e.g. trypsin, chymotrypsin, plasmin, } \\
\text { thrombin }\end{array}$ \\
\hline EDTA-Na ${ }_{2}$ & metalloproteases \\
\hline Aprotinin & serine proteases \\
\hline
\end{tabular}


After incubation of the cells for 48 hours with the inhibitors, the lysate was harvested and protein content determined by Bradford assay. Afterwards, the lysate was applied to SDS-polyacrylamide-gelelectrophoresis and analyzed by Western Blot using the $\alpha \mathrm{F}_{\text {ecto-antibody. }}$ -

No cleavage inhibition was achieved with the inhibitors phosphoramidon, EDTA-Na, bestatin, pepstatin and aprotinin, the $\mathrm{F} 1$ and the $\mathrm{F} 1 \mathrm{a}$ fragment were still detectable in high amounts and in addition to the F0 fragment (Fig. 24). In contrast, reduced amounts of $\mathrm{F} 1$ and F1a were observed when transfected cells were incubated with the inhibitors antipain, E-64 and leupeptin and possibly chymostatin. Incubation with Pefabloc SC resulted in cell death, thus, no analysis of the effect of this inhibitor on protease cleavage was possible.
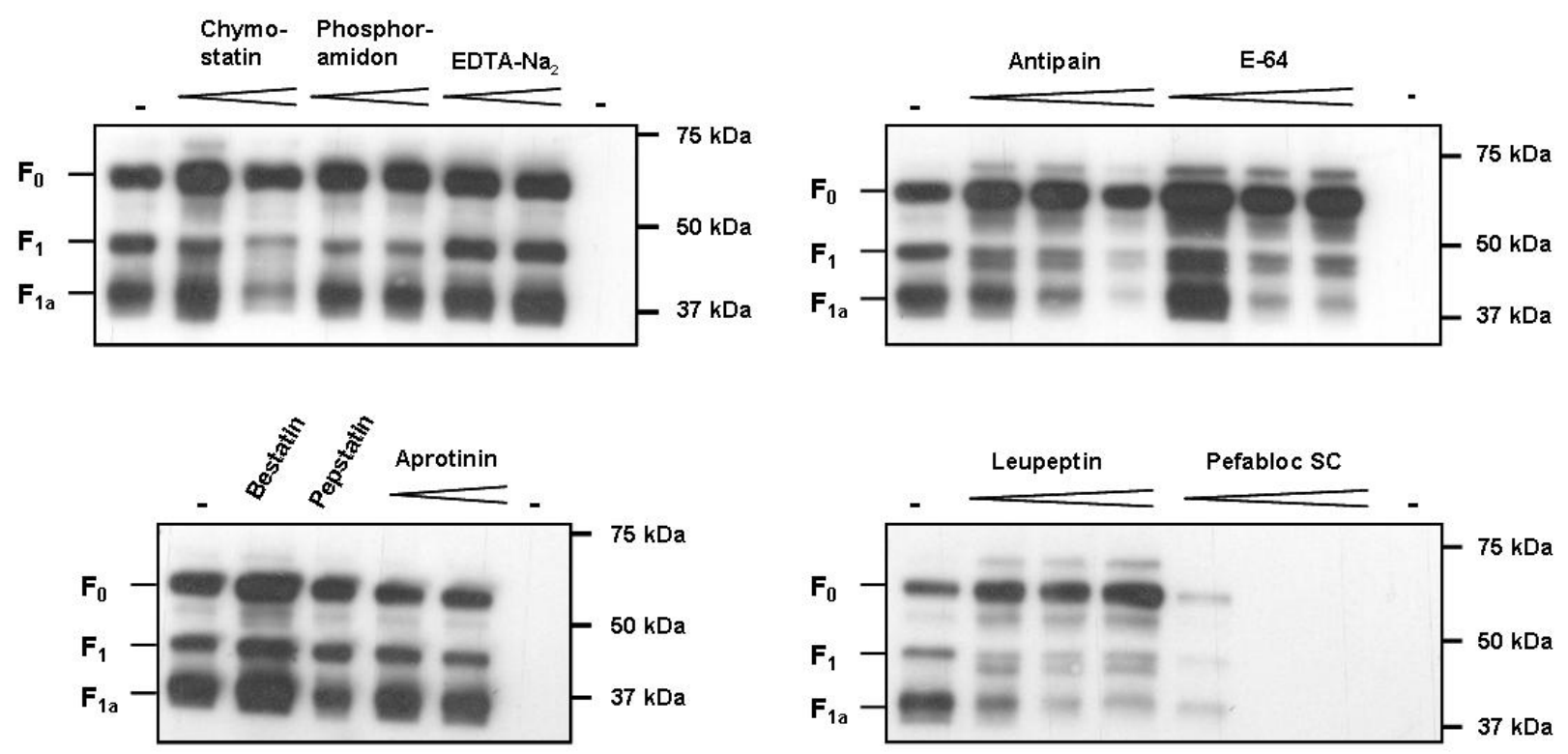

Figure 24: Screening of ten different protease inhibitors

293T cells were transfected with TPMV F and five hours after transfection the medium was replaced by medium supplemented with the respective protease inhibitors in different concentrations suggested by the manufacturer. 48 hours later, the lysate was harvested and proteins were separated by SDS$10 \%$ PAGE followed by Western Blot analysis using the antibody $\alpha \mathrm{F}_{\text {ecto. }}$. The positions of F0, F1 and F1a are indicated on the left.

Having demonstrated the effects of different protease inhibitors in this first screening, now the potentially effective inhibitors chymostatin, antipain, E-64 and leupeptin were selected and tested in several concentrations, starting with lowest concentration suggested by the manufacturer. Lysate samples were prepared as described before followed by Western Blot analysis. 
In the presence of leupeptin, no cleavage occurred, neither for F1/F2 nor F1a/F1b, even with the lowest concentration of $1 \mu \mathrm{g} / \mathrm{ml}$ only the F0 fragment was detectable (Fig. 25, A). Antipain inhibited protease cleavage with increasing concentrations (Fig. 25 , B). With higher amounts of inhibitor higher amounts of the F0 fragment were detected. The F1 fragment is less, but independently of the inhibitor concentration expressed, whereat there was almost no F1a expressed after incubation with 200 and $400 \mu \mathrm{g} / \mathrm{ml}$. A similar effect was observed for E-64 (Fig. 25, D). Incubation with chymostatin with concentrations of 25 and $50 \mu \mathrm{g} / \mathrm{ml}$ resulted in detection of F0, F1 and F1a fragments (Fig. 25, C) demonstrating that cleavage is not inhibited.

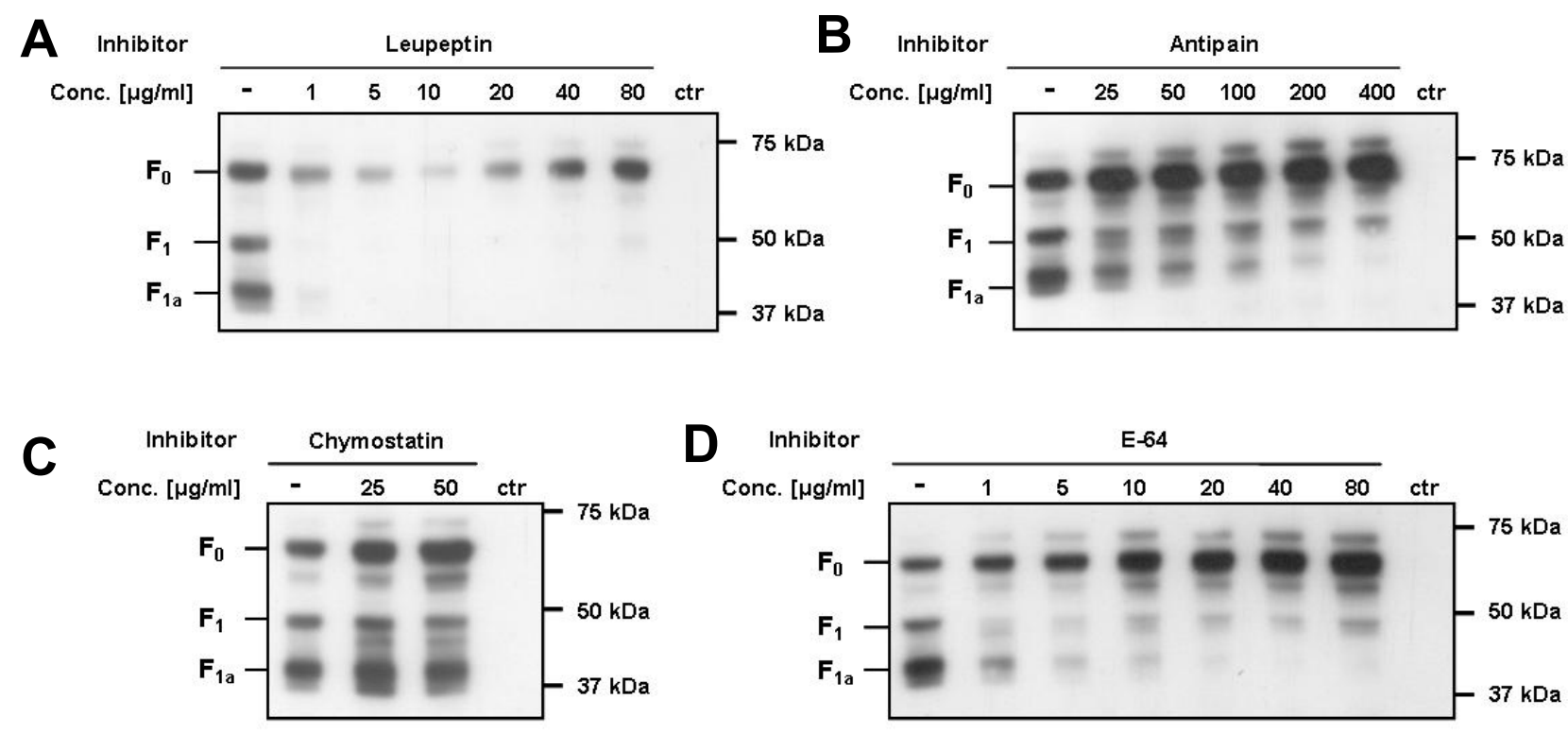

Figure 25: $F$ protein cleavage is inhibited by leupeptin, antipain and E-64

To further characterize the promising protease inhibitors leupeptin (A), antipain (B), chymostatin (C) and E-64 (D), 293T cells were transfected with TPMV $F$ and five hours after transfection the medium was replaced by medium supplemented with stepwise diluted protease inhibitor, respectively. 48 hours later, the lysate was harvested and proteins were separated by SDS-10\% PAGE followed by Western Blot analysis using the antibody $\mathrm{aF}_{\text {ecto. }}$. The positions of F0, F1 and F1a are indicated on the left.

The inhibitors and the respective protease families are summarized in Table 2. Taken together, all effective substances (Leupeptin, Antipain, E-64) inhibited the family of cysteine proteases. These data suggest that a cysteine protease might be involved in $\mathrm{F}$ protein cleavage and activation. 
Table 2: Protease families and their inhibitors

\begin{tabular}{|c|c|c|c|c|c|c|}
\hline & Aspartic & Cysteine & Metallo & Serine & Threonine & $\begin{array}{c}\text { Inhibition } \\
\text { of TPMV F } \\
\text { cleavage }\end{array}$ \\
\hline Antipain & & $\mathrm{x}$ & & $\mathrm{x}$ & & + \\
\hline Bestatin & & & $\mathrm{x}$ & & & - \\
\hline Chymostatin & & $\mathrm{x}$ & & $\mathrm{x}$ & & - \\
\hline E-64 & & $\mathrm{x}$ & & & & + \\
\hline Leupeptin & & $\mathrm{x}$ & & $\mathrm{x}$ & $\mathrm{x}$ & + \\
\hline Pepstatin & $\mathrm{x}$ & & & & & - \\
\hline Phosphoramidon & & & $\mathrm{x}$ & & & - \\
\hline Pefabloc SC & & & & $\mathrm{x}$ & & - \\
\hline EDTA-Na 2 & & & $\mathrm{x}$ & & & - \\
\hline Aprotinin & & & & $\mathrm{x}$ & & - \\
\hline
\end{tabular}

\subsection{2. $F$ protein cleavage is necessary for cell fusion}

To answer the question if $F 1 / F 2$ and $F 1 a / F 1 b$ cleavage is necessary for cell fusion the effect of the three identified protease inhibitors leupeptin, antipain and E-64 were examined in fusion assays. TBF cells were transiently transfected with TPMV-H- and -F-expression plasmids. Five hours after transfection the medium was replaced by medium supplemented with the protease inhibitors at different concentrations, respectively, and as control, medium without inhibitor was added. Fourty-eight hours later, the cells were observed under the microscope and the lysate was harvested. Afterwards, the protein content was determined by Bradford assay and the lysate applied to SDS-polyacrylamide-gelelectrophoresis and analyzed by Western Blot.

As expected, TBF cells transfected with pCG-TPMV-H and pCG-TPMV-F fused and form large syncytia after incubation with non-supplemented medium (Fig. 26). 

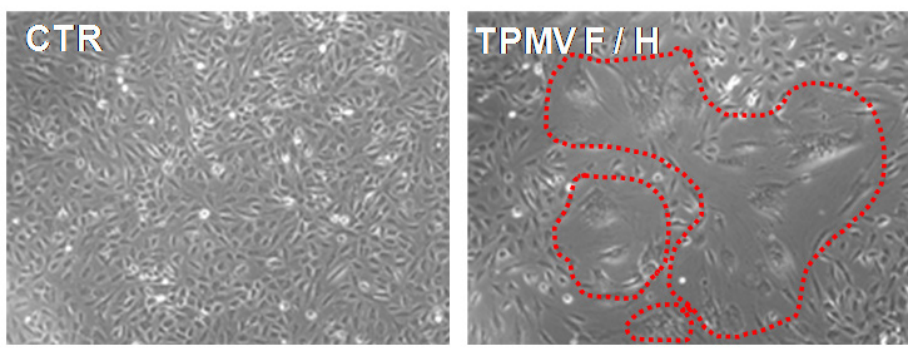

Figure 26: TBF cells form large syncytia after transfection with TPMV $H$ and $F$

TBF cells were transfected with expression plasmids for TPMV H and F. Fourty-eight hours after, cells were analyzed by microscopy using 10x magnification and representative pictures were taken. Left: ut, untransfected cells; right: cells transfected with TPMV F and $\mathrm{H}$.

In TPMV-H and -F-transfected TBFs incubated with antipain-supplemented medium $(100 \mu \mathrm{g} / \mathrm{ml}$ and $400 \mu \mathrm{g} / \mathrm{ml}$ ) fusion of cells was completely inhibited (Fig. 27). For E-64 and Leupeptin, a complete inhibition of cell fusion was only observed when cells were incubated with higher inhibitor concentrations $[80 \mu \mathrm{g} / \mathrm{ml}]$.

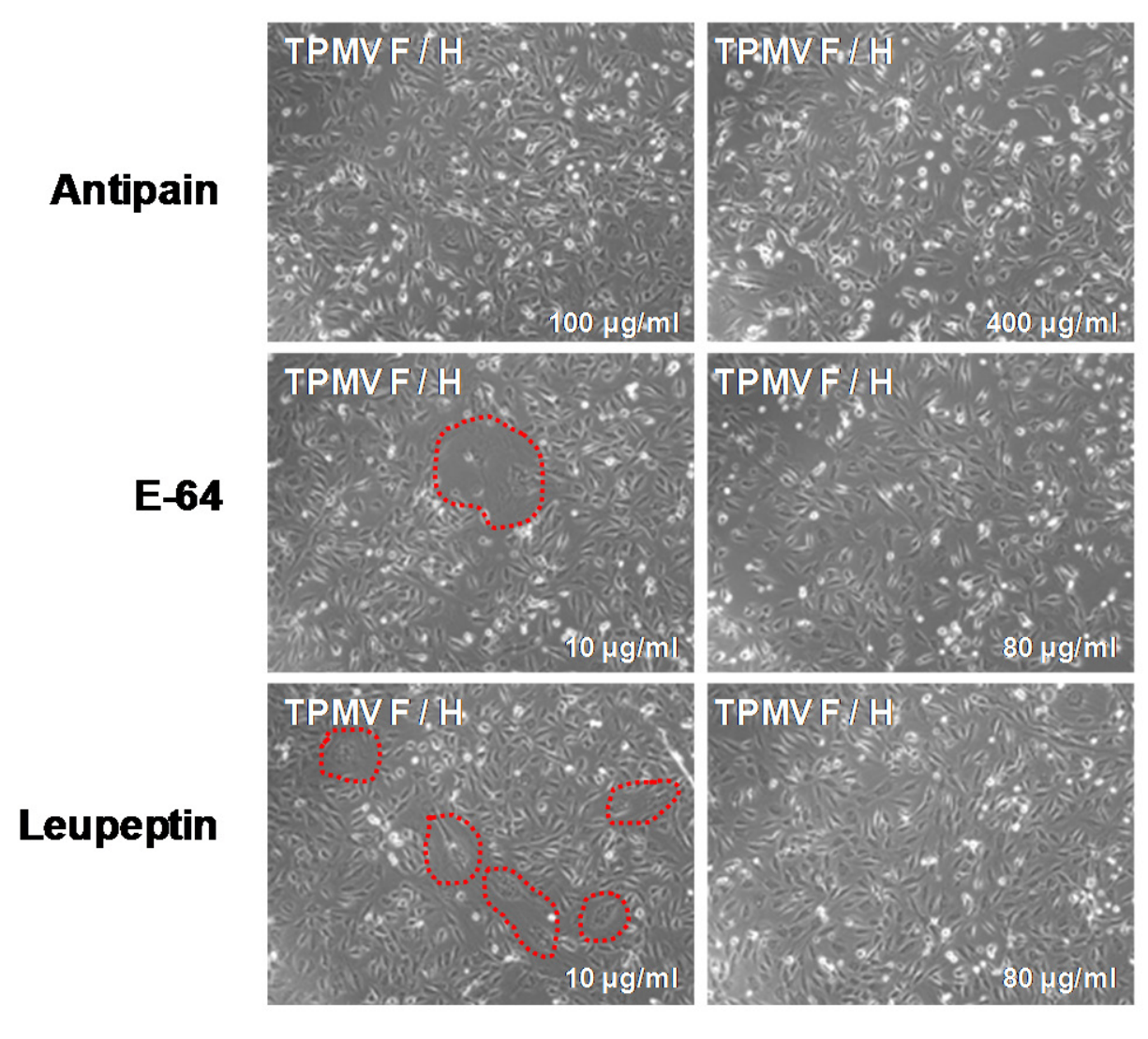

\section{Inhibitor concentration}

Figure 27: Protease inhibitors prevent syncytia formation of TPMV F/H-transfected TBF cells TBF cells were transfected with expression plasmids for TPMV H and F. Five hours after transfection, the medium was replaced by medium supplemented with the protease inhibitors antipain, E-64 or leupeptin in two different concentrations. 48 hours later, cells were analyzed by microscopy using $10 \mathrm{x}$ magnification and representative pictures were taken. 


\subsection{Pseudotyping of lentiviral vectors with the modified TPMV proteins}

Characterization of the modified TPMV glycoproteins showed that all variants were expressed after transient transfection. Additionally, modified TPMV $\mathrm{H}$ proteins that are expressed on the cell surface and have fusion helper function were identified, as well as $\mathrm{F}$ proteins with truncated cytoplasmic tails, that still support fusion of the cell membrane. The next step for generating retargeted lentiviral vectors was to pseudotype them with the modified TPMV glycoproteins.

\subsubsection{Generation of TPMV-pseudotyped vectors}

In a first screening, the untruncated TPMV HaCD20 protein was tested together with all $F$ protein variants for the ability to pseudotype lentiviral vectors. For the production, $293 \mathrm{~T}$ cells served as packging cells and were transiently transfected in a T75 flask with the packaging plasmid pCMV $\Delta R 8.9$, the green fluorescent protein (GFP) encoding transfer vector plasmid pSEW, the TPMV HaCD20 plasmid and one of the TPMV F variant-encoding plasmids, respectively. The TPMV-plasmids were used in equal amounts. As positive control, VSV-G-pseudotyped vectors were generated by transfection of the VSV-G encoding plasmid pMD.G2 along with the packaging and transfer plasmids. Additionally, vector particles that are pseudotyped with $M V_{N S e} H \Delta 18 \alpha C D 20$ and $F \Delta 30$ (MV vaccine strain Edmonston $B$ ) or $M V_{W T} H \Delta 18$ and $\mathrm{F} \Delta 30$ (MV wildtype strain) (Funke et al., 2008a) were produced. Fourty-eight hours after transfection, the supernatants of vector-producing 293T cells were harvested, concentrated by low-speed centrifugation and used for transduction of the CD20-positive cell line Raji (Human Burkitt's lymphoma cell line). After 72 hours, the transduced cells were analyzed by FACS (fluorescent activated cell sorting) and the titers were calculated.

Efficiency of transfection was analyzed by fluorescence microscopy. The packaging cells showed high GFP expression due to the pSEW plasmid (Fig. 28). For both MV and TPMV vector producing cells, syncytia formation was observed that was possibly due to the high transfection levels and aCD20-scAb interactions. In MV-vector producing cells, syncytia formation was increased, probably because of incomplete mutation of the MV H contact residues to the MV receptors. 

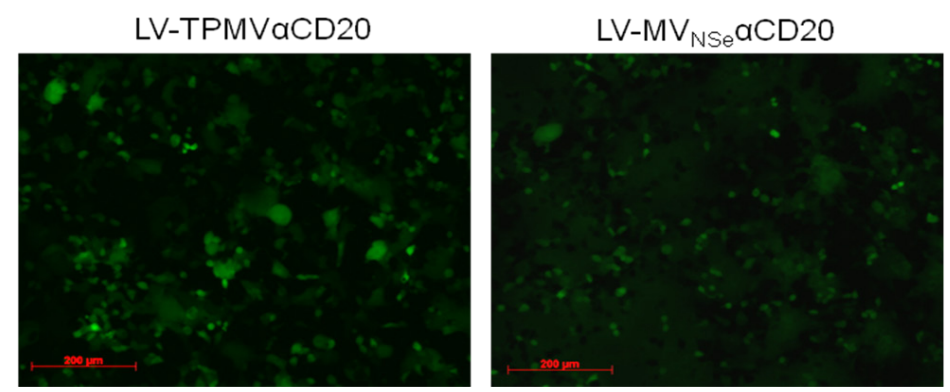

Figure 28: GFP expression of packaging cells after plasmid transfection for LV generation 293T packaging cells were co-transfected with lentiviral expression plasmids and plasmids encoding TPMV HaCD20 and $F(L V-T P M V a C D 20)$ or $M_{\text {NSe }} H \Delta 18 \alpha C D 20$ and $F \Delta 30$ (LV-MV NSe $_{\text {e }} \alpha C D 20$ ), respectively. Fourty-eight hours after transfection, GFP expression was analyzed by fluorescence microscopy (100x magnification). Representative pictures are shown. Scale bar: $200 \mu \mathrm{m}$

Seventy-two hours after transduction of Raji cells, highest titers of the TPMVpseudotypes were achieved with HaCD20 together with the unmodified F, FA18 and F $\Delta 32\left(9 \times 10^{5}-3 \times 10^{6}\right.$ t.u./ml) (Fig. 29). These values are almost in the same range as the $\mathrm{MV}_{\mathrm{NSe}}$-control $\left(3.5 \times 10^{6}\right.$ t.u. $\left./ \mathrm{ml}\right)$. Higher titers were only reached with $\mathrm{MV}_{\mathrm{WT}}$

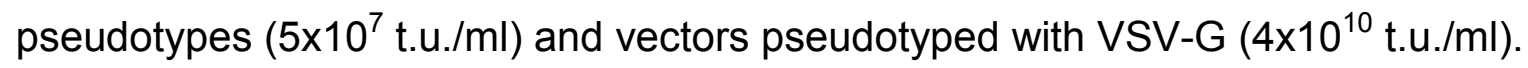

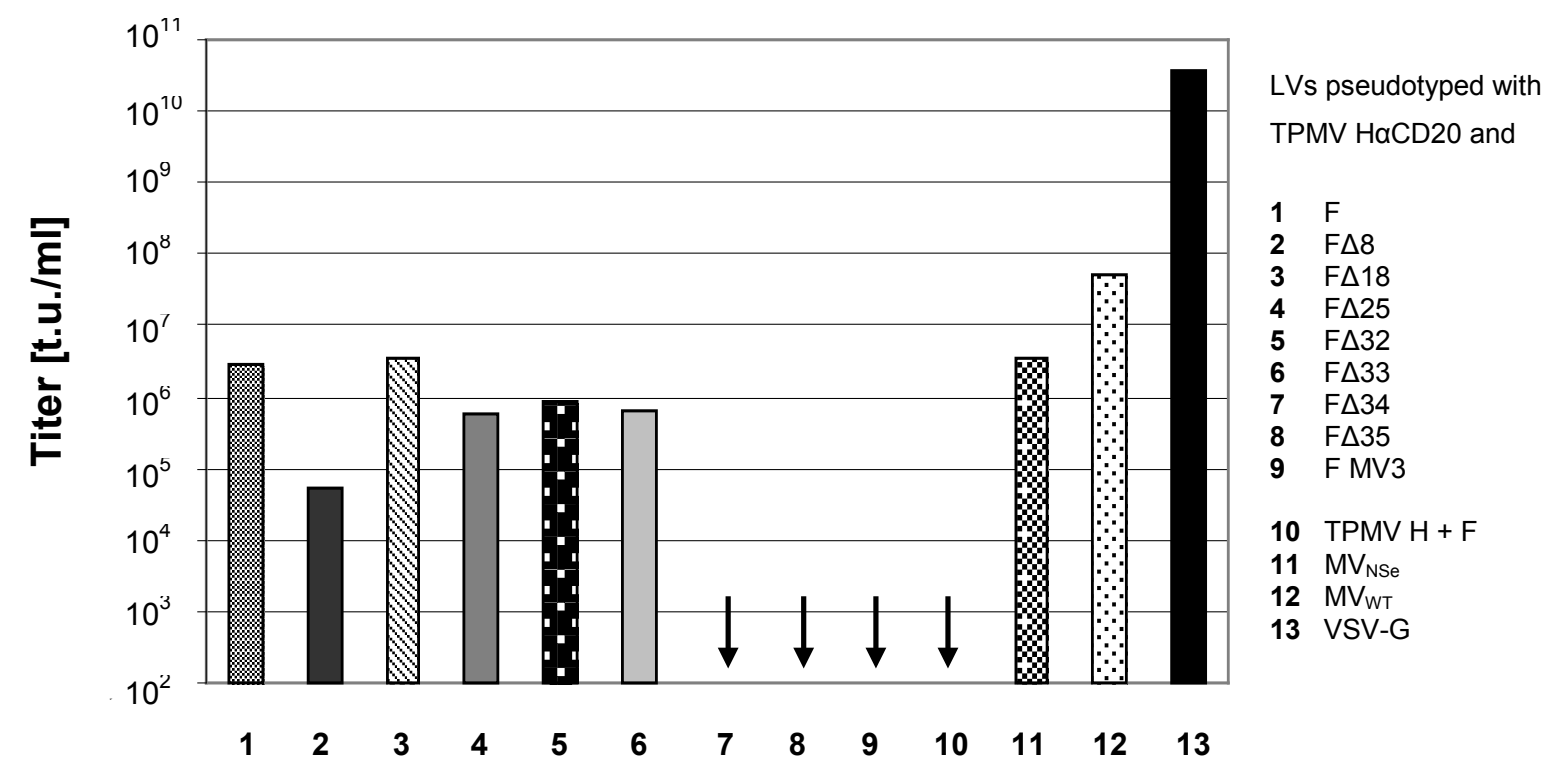

Figure 29: Initial screening titers of TPMV HaCD20 and F protein variants to pseudotype LVs $293 T$ cells were co-transfected with lentiviral expression plasmids and plasmids encoding TPMV $\mathrm{HaCD} 20$ and different truncated $\mathrm{F}$ variants, respectively. After 48 hours, vector-containing supernatant was harvested and concentrated by low-speed centrifugation for 24 hours at $4^{\circ} \mathrm{C}$. The pellet was then used for transduction of Raji cells. Seventy-hours later, GFP expression was analyzed by FACS and titers [t.u. $/ \mathrm{ml}$, titration units $/ \mathrm{ml}$ ] were determined. The arrows indicate titers below the detection limit. $M_{N S e}$ : glycoproteins of measles virus vaccine strain Edmonston $B, H \Delta 18 \alpha C D 20$ and $F \Delta 30 ; M_{W T}$ : glycoproteins of measles virus wild type strain, $\mathrm{H} \Delta 18$ and $\mathrm{F} \Delta 30$; VSV-G: glycoprotein of vesicular stomatitis virus; GFP: green fluorescent protein; LVs: lentiviral vectors 
Surprisingly, TPMV HaCD20 together with untruncated $F$ allowed efficient pseudotype formation $\left(3 \times 10^{6}\right.$ t.u.ml). This is in contrast to the MV-pseudotyped vectors, where only truncated MV glycoproteins could be incorporated (Funke et al., 2008a). In accordance with the results obtained in syncytia assays, F proteins with a cytoplasmic tail shorter than 5 amino acids are not able to mediate gene transfer, as well as the TPMV $\mathrm{H}$ protein without the aCD20-scAb.

In further experiments, $M V_{\text {NSe }}$ truncated and retargeted glycoproteins (H $\triangle 18 \mathrm{aCD} 20$ and $\mathrm{F} \Delta 30$ ) are used as a control and will be referred to as LV-MVaCD20.

\subsubsection{Screening of all TPMV $H$ and $F$ protein variants}

Next, all TPMV $\mathrm{H}$ and $\mathrm{F}$ protein variants were screened in all combinations for their ability to mediate gene transfer into CD20-positive cells. Since it is associated with high effort to test all combinations in T75 flasks, this screening was performed in a 6 well format.

Vectors were produced by transfection of 293T cells with lentiviral expression plasmids and plasmids encoding the TPMV glycoproteins in equal amounts as described above, but with corresponding less plasmid DNA and in a 6 well plate. Two days post transfection, the supernatant of the packaging cells was directly used for transduction of CD20-positive Raji cells in a 96 well plate. After 48-72 hours, transduced cells showed GFP expression and were analyzed by FACS. Based on the percentage of GFP-expressing cells the titer (transducing units (t.u.)/ml) was calculated.

Titers obtained by screening all $\mathrm{F}$ and $\mathrm{H}$ protein combinations are shown in Table 3 . 
Table 3: Screening titers of TPMV H and F combinations for LV-pseudotyping

\begin{tabular}{|c|c|c|c|c|c|c|c|c|c|}
\hline $\begin{array}{l}\text { TPMV }^{\mathrm{a}} \\
\text { [t.u./ml] }\end{array}$ & HaCD20 & $\mathrm{H} \Delta 20 \mathrm{\alpha CD} 20$ & $\mathrm{H} \Delta 40 \mathrm{aCD} 20$ & $\mathrm{H} \Delta 50 \mathrm{aCD} 20$ & $\mathrm{H} \Delta 60 \mathrm{aCD} 20$ & $\mathrm{H} \Delta 70 \mathrm{aCD} 20$ & $\mathrm{H} \Delta 80 \alpha \mathrm{CD} 20$ & $H \Delta 84 \alpha C D 20$ & TPMV-H \\
\hline $\mathbf{F}$ & $4.0 \mathrm{E}+04$ & $2.9 E+03$ & $4.9 E+03$ & $4.6 \mathrm{E}+03$ & $2.3 E+04$ & $6.3 \mathrm{E}+04$ & $5.2 \mathrm{E}+04$ & $1.4 \mathrm{E}+04$ & $<1.3 \mathrm{E}+03$ \\
\hline$F \Delta 8$ & $8.9 E+03$ & $<1.3 \mathrm{E}+03$ & $<1.3 \mathrm{E}+03$ & $1.8 \mathrm{E}+04$ & $1.9 \mathrm{E}+04$ & $4.6 \mathrm{E}+03$ & $5.4 \mathrm{E}+04$ & $1.8 \mathrm{E}+04$ & $<1.3 \mathrm{E}+03$ \\
\hline F $\Delta 18$ & $4.2 \mathrm{E}+04$ & $2.9 E+03$ & $1.3 E+04$ & $1.8 \mathrm{E}+04$ & $3.4 \mathrm{E}+04$ & $1.1 \mathrm{E}+04$ & $8.9 E+04$ & $4.9 \mathrm{E}+04$ & $<1.3 \mathrm{E}+03$ \\
\hline $\mathrm{F} \Delta 25$ & $6.1 \mathrm{E}+04$ & $7.4 \mathrm{E}+03$ & $6.0 \mathrm{E}+04$ & $1.9 \mathrm{E}+03$ & $2.1 \mathrm{E}+04$ & $2.6 \mathrm{E}+04$ & $8.4 \mathrm{E}+04$ & $5.9 E+04$ & $<1.3 \mathrm{E}+03$ \\
\hline $\mathrm{F} \Delta 32$ & $3.1 \mathrm{E}+04$ & $5.4 \mathrm{E}+03$ & $8.4 \mathrm{E}+04$ & $1.7 \mathrm{E}+03$ & $6.9 \mathrm{E}+04$ & $2.9 \mathrm{E}+04$ & 1.3E+05 & $9.9 \mathrm{E}+04$ & $<1.3 \mathrm{E}+03$ \\
\hline$F \Delta 33$ & $2.0 \mathrm{E}+04$ & $<1.3 \mathrm{E}+03$ & $1.5 \mathrm{E}+04$ & $3.4 \mathrm{E}+03$ & $1.3 \mathrm{E}+04$ & $3.5 \mathrm{E}+03$ & $2.3 E+04$ & $6.2 \mathrm{E}+03$ & $<1.3 \mathrm{E}+03$ \\
\hline $\mathrm{F} \Delta 34$ & $<1.3 \mathrm{E}+03$ & $<1.3 \mathrm{E}+03$ & $<1.3 \mathrm{E}+03$ & $<1.3 \mathrm{E}+03$ & $<1.3 \mathrm{E}+03$ & $<1.3 \mathrm{E}+03$ & $<1.3 \mathrm{E}+03$ & $<1.3 \mathrm{E}+03$ & $<1.3 \mathrm{E}+03$ \\
\hline F $\Delta 35$ & $<1.3 \mathrm{E}+03$ & $4.3 E+03$ & $<1.3 \mathrm{E}+03$ & $<1.3 \mathrm{E}+03$ & $<1.3 \mathrm{E}+03$ & $<1.3 \mathrm{E}+03$ & $<1.3 \mathrm{E}+03$ & $<1.3 \mathrm{E}+03$ & $<1.3 \mathrm{E}+03$ \\
\hline F MV3 & 2.6E+03 & $<1.3 \mathrm{E}+03$ & $3.4 \mathrm{E}+03$ & $8.1 \mathrm{E}+03$ & 1.7E+03 & $<1.3 \mathrm{E}+03$ & $<1.3 \mathrm{E}+03$ & $<1.3 \mathrm{E}+03$ & $<1.3 \mathrm{E}+03$ \\
\hline
\end{tabular}

\begin{tabular}{|l|}
\hline detection limit \\
\hline$<1.3 \mathrm{~b}+03$ \\
\hline $1.3 \mathrm{E}+03-1.00 \mathrm{E}+03$ \\
\hline $1.0 \mathrm{E}+04-1.0 \mathrm{E}+05$ \\
\hline $1.0 \mathrm{E}+05$ \\
\hline
\end{tabular}

${ }^{\text {a }}$ screening titers in transducing units $/ \mathrm{ml}$ [t.u./ml];

screening was performed in duplicate

${ }^{b}$ detection limit: detection of $>1 \%$ GFP expression in FACS analysis

Highest titers $\left(10^{5}\right.$ t.u. $\left./ \mathrm{ml}\right)$ were reached with the truncated $\mathrm{F}$ variant $\mathrm{F} \Delta 32$, where only six amino acids of the cytoplasmic tail were left, together with $H \triangle 80 a C D 20$ that has a cytoplasmic tail length of 14 aa (Table 3 ). High titers were also obtained with the combinations $\mathrm{H} \Delta 80 \mathrm{aCD} 20$ / $\mathrm{F} \Delta 18$ and $\mathrm{H} \Delta 84 \alpha \mathrm{aCD} 20$ / $\mathrm{F} \Delta 32\left(9.8 \times 10^{4}\right.$ and $9.9 \mathrm{x}$ $10^{4}$ t.u. $/ \mathrm{ml}$ ). Concentration of pseudotyped vectors produced in large scale (T75 or T175 flask) by low speed centrifugation increased titers up to about $10^{6} \mathrm{t}$.u. $/ \mathrm{ml}$. Altogether, there was a trend to higher titers when glycoproteins with shorter cytoplasmic tails were used. However, F proteins with cytoplasmic tails shorter than five amino acids did not lead to infectious vectors. Considering the loss of fusogenicity of these proteins in fusion assays, this result was expected and already observed in a first screening (Fig. 18). In this screening it was also shown that in contrast to MV, it was possible to pseudotype lentiviral vectors with unmodified TPMV $\mathrm{H}$ and $\mathrm{F}$ proteins although titers were low. Untargeted TPMV $\mathrm{H}$ protein was used as a negative control, truncated MV glycoproteins (Funke et al., 2008a) were used as a positive control and led on average to vector titers in the range of 2-3 $\times 10^{4}$ t.u./ml (data not shown). VSV-G-pseudotyped vectors reached titers much higher than $10^{6}$ t.u. $/ \mathrm{ml}$ that were above the detection limit in this experiment. The optimal TPMV $\mathrm{F}$ and $\mathrm{H}$ combination identified in this screening was used for all further experiments $(\mathrm{H} \triangle 80 \mathrm{aCD} 20$ together with $\mathrm{F} \Delta 32)$ and will be referred to as LVTPMVaCD20. 


\subsubsection{Determination of the optimal $H$ to $F$ ratio}

In natural occurring MV infections, it was shown, that more F mRNA than H mRNA is produced (Cattaneo et al., 1987a; Plumet, Duprex, and Gerlier, 2005). Following this observation, the $\mathrm{H}$ to $\mathrm{F}$ ratio of MV-pseudotyped vectors was optimized, which resulted in a more than ten-fold increase in titer. The optimal ratio of $\mathrm{H}$ to $\mathrm{F}$ identified for MV was $1: 7$ (Funke et al., 2008a). Since there is a gradient of mRNA in all paramyxoviruses, it was tried to enhance titers of TPMV-pseudotyped by optimization the TPMV H $\triangle 80$ aCD20 to $\mathrm{F} \Delta 32$ plasmid ratio.

For this purpose, vectors were produced by co-transfection of 293T cells in T75 flasks with the lentiviral expression plasmids and different ratios of pCG-TPMV$\mathrm{H} \triangle 80 \mathrm{aCD} 20$ and pCG-TPMV-F $\Delta 32$, respectively. After 48 hours, the cell supernatants were harvested and concentrated for 24 hours by low-speed centrifugation. The concentrated particles were then used for transduction of Raji cells. Seventy-two hours later, GFP expression was analyzed by FACS and titers were calculated. The titers were normalized to that obtained after transfection of equal amounts of pCG-TPMV-H $\triangle 80 \mathrm{aCD} 20$ and pCG-TPMV-F $\Delta 32$.

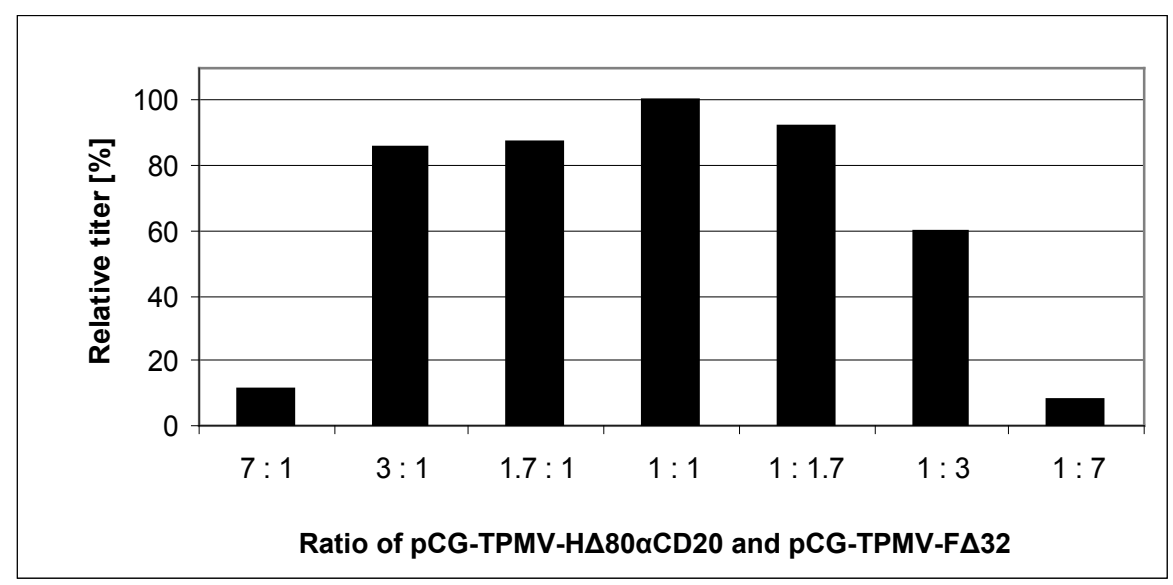

Figure 30: Identification of optimal H : F ratio for pseudotyping lentiviral vectors

During vector production, the plasmid ratio of $H \triangle 84 a C D 20$ to $F \Delta 32$ was altered as indicated. Vectors were titrated on Raji cells and titers determined by FACS analysis. Titers were normalized to that obtained after transfection with equal amounts of TPMV H $\triangle 84 a C D 20$ and F $\Delta 32$ expression plasmids $(100 \%)$. This figure shows the average of 3 independently experiments.

In contrast to MV-pseudotypes, there was no significant enhancement of titers by altering the ratio of $\mathrm{H} \triangle 80 \mathrm{aCD} 20$ and $\mathrm{F} \triangle 32$ plasmids during vector particle production (Fig. 30). The data show the average of 3 independent experiments. Titers obtained 
with equal amounts of $\mathrm{H}$ and $\mathrm{F}$ plasmids were slightly higher than that obtained with other $\mathrm{H}$ : $\mathrm{F}$ ratios. Using lesser amounts of $\mathrm{H}$ or $\mathrm{F}$ plasmids, respectively, resulted in a decrease of titers. Compared to MV-pseudotypes, where a ratio of $1: 7$ is optimal for production, in this case, in a ratio of $1: 7$ and vice versa the titers were decreased to less than $15 \%$. Further experiments were performed in a $\mathrm{H} \Delta 80 \mathrm{aCD} 20$ and $\mathrm{F} \Delta 32$ plasmid ratio of $1: 1$.

\subsubsection{Confirmation of incorporation of the modified TPMV proteins into LVs}

Having demonstrated that lentiviral vectors pseudotyped with truncated TPMV glycoproteins mediated efficient gene transfer, now the incorporation level of the gylcoproteins was investigated.

For this purpose, TPMVaCD20 vectors were generated as described before, concentrated and purified by low speed centrifugation over a $20 \%$ sucrose cushion for $24 \mathrm{~h}$ at $4^{\circ} \mathrm{C}$. The vectors were resuspended in PBS, inactivated for 5 minutes at $95^{\circ} \mathrm{C}$ and their protein content determined by Bradford assay. As a control, 293T cells were transfected with $\mathrm{H} \Delta 80 \mathrm{aCD} 20$ - or F $\Delta 32$-plasmids. After 48 hours, the cells were lysed and the protein content determined. The purified particles and the cell lysates were applied to SDS-polyacrylamide-gelelectrophoresis and analyzed by Western Blot.

Both glycoproteins were detectable in the particles and migrated according to their molecular size albeit $H \Delta 80 a C D 20$ was barely detected (Fig. 31, lane 1). A stronger signal was observed for $F \Delta 32$ and the fragments $F 0$ and $F 1$ were detectable whereat $\mathrm{F}$ 1a seemed not to be present. This observation was very different from the $\mathrm{F}$ protein fragments present in TPMV virions (section 6.2.4).

In particles containing only $H \Delta 80 \alpha C D 20$ but no $F \Delta 32$, stronger signals for the $H$ protein were observed (lane 2). The p24 protein, that is part of the HIV-1 capsid, could be detected in all four vector productions, demonstrating, that particles are also produced in the absence of any glycoproteins (lane 4). Analysis of p24 suggested an increased protein expression level compared to TPMV glycoproteins. 


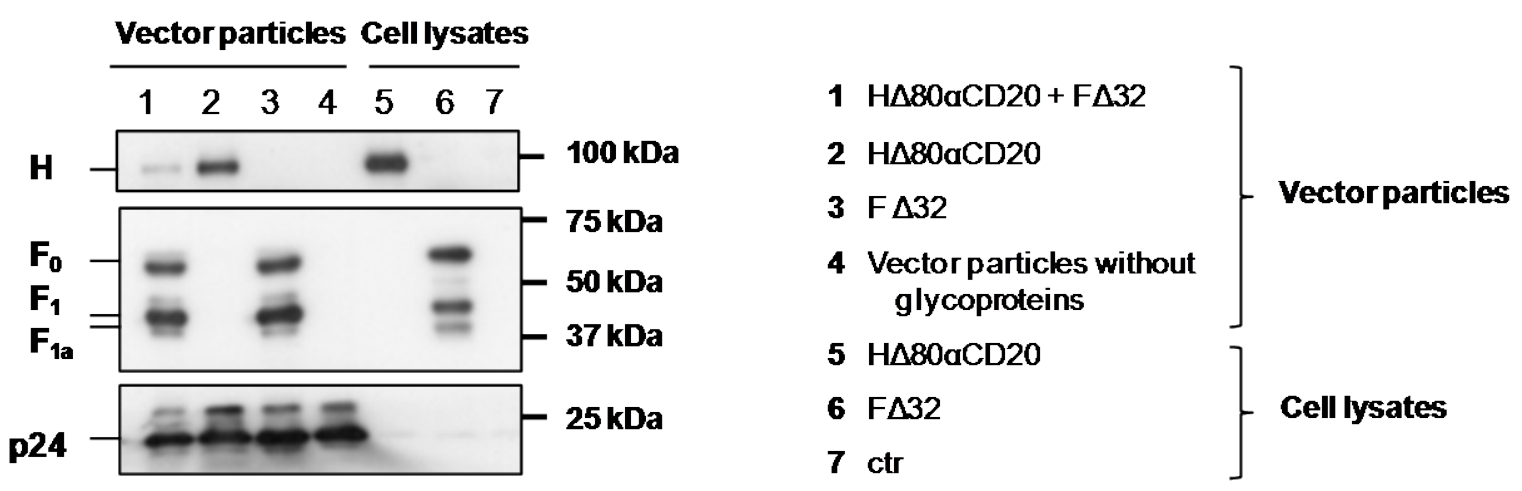

Figure 31: Biochemical confirmation of assembly of TPMV-pseudotyped lentiviral vectors Concentrated vector particles were analyzed by Western Blot using aHecto, aFecto and ap24antibody, respectively. As control, cell lysates of $\mathrm{H} \Delta 84 \alpha \mathrm{CD} 20, \mathrm{~F} \Delta 32$ or untransfected (ut) cells were used. The positions of TPMV H, F0, F1, F1a and p24 are indicated on the left.

\subsubsection{Targeting of different CD20-positive and negative cell lines}

After characterization of the TPMV-pseudotyped vectors, their targeting potential was investigated. For this purpose, the cell lines 293T, HT1080, HT1080 that stable express CD20 (HT1080-CD20) and Tupaia baby fibroblasts (TBFs) were transduced immediately after production of the respective vector types with LV-TPMVaCD20, a TPMV-pseudotyped vector (TPMV $\mathrm{H} \Delta 80, \mathrm{~F} \Delta 32$ ) that did not carry a scAb on the $\mathrm{H}$ protein (TPMV (w/o scAb)) and as controls, with LV-MVaCD20 and LV-VSV-G. After 72 hours, GFP expression was analyzed by fluorescence microscopy and representative pictures were taken.

Remarkably, LV-TPMVaCD20 showed no background transduction of the receptor negative cell lines 293T and HT1080 but as expected, TBF cells were transduced (Fig. 32). HT1080 cells that stable express CD20 (HT1080-CD20) were also transduced, demonstrating that cell entry occurs via the aCD20-scAb. The TPMVpseudotyped vector without a scAb only transduced the TPMV-native cell line TBF, indicating the lack of a receptor for TPMV on human cells. GFP expression in TBF cells is lower compared to GFP expression of HT1080-CD20 cells transduced by LVTPMVaCD20, for example.

Some background transduction could be observed for LV-MVaCD20, suggesting that mutation of contact residues was incomplete or that vectors entered cells via the recently identified new MV receptor nectin-4 (Noyce et al., 2011) for which the H protein is not blinded yet. Apart from that, this vector type also entered cells primary 
via aCD20-scAb, demonstrated by transduction of HT1080-CD20 cells. As expected, VSV-G-pseudotyped vectors entered all cell lines tested with high transduction efficiencies.

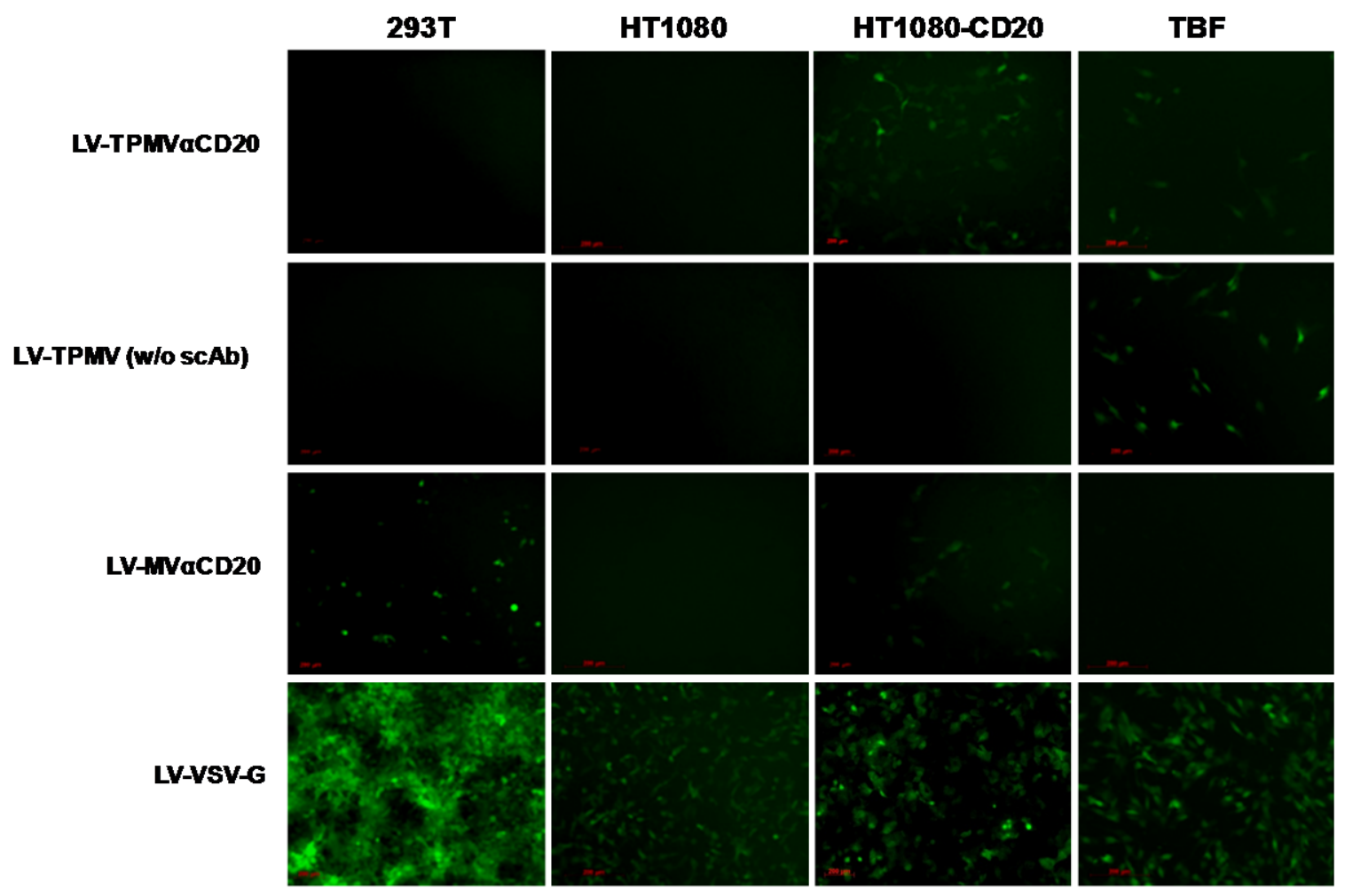

Figure 32: GFP expression of different cell lines transduced with the indicated vector types 293T cells, HT1080 cells, HT1080 cells stable expressing CD20 (HT1080-CD20) and Tupaia baby fibroblasts were transduced with LV-TPMVaCD20, LV-TPMV(w/o scAb) (TPMV F $\Delta 32$ and $H \Delta 80$ without $\mathrm{scAb}$ ) and LV-VSV-G. After 72 hours, GFP expression was analyzed by fluorescence microscopy (100x magnification). Representative pictures are shown. Scale bar: $200 \mu \mathrm{m}$

\subsection{Stability assays of TPMV-pseudotyped lentiviral vectors}

Up to now, the TPMV-pseudotyped vectors were concentrated by low-speed centrifugation and used for experiments immediately after production. But for some experiments it might be necessary to produce high amounts of vectors and to store them until needed. To investigate the stability of the new vectors and how to maintain their functionality, different production and storage conditions were tested. 


\subsubsection{Concentration of vector particles}

In a first step, different concentration methods of vector particles were investigated and the recovery calculated. The recovery indicates how many functional vector particles get lost during concentration. Since low speed centrifugation for 24 hours is very time-consuming, two more concentrating techniques were tested. The vectors were produced as described before and $15 \mathrm{ml}$ cell supernatant, respectively, concentrated by low speed centrifugation (LS) for $24 \mathrm{~h}$ at $4000 \mathrm{~g}$, ultra centrifugation (UC) for 3 hours at $28000 \mathrm{~g}$ over a $20 \%$ sucrose cushion or by ultra filtration (UF) for 2 hours at $4000 \mathrm{~g}$. Pellets containing viral vectors were resuspended in $120 \mu \mathrm{l} \mathrm{RPMl}$ for concentrating by LS and UC, by ultra filtration the volume achieved was $240 \mu \mathrm{l}$.

For LV-TPMVaCD20 and LV-MVaCD20, highest recovery was achieved by low speed centrifugation ( $48.5 \%$ and $82.0 \%$, Table 4 ) although there was a loss of more than $50 \%$ of infectious vector particles for TPMV-pseudotypes. Concentration by ultra centrifugation resulted in a recovery of only $6.6 \%$ for LV-TPMVaCD20 and 39.2 $\%$ for LV-MVaCD20. Regarding the concentrating technique of ultra filtration, there was a very low recovery observed for LV-TPMVaCD20 (5.4\%) but for LV-MVaCD20, the recovery was $73.5 \%$ which was almost as high as for low speed centrifugation, respectively.

Table 4: Titer and recovery of vectors after different concentration methods

\begin{tabular}{|c|c|c|c|c|c|c|}
\hline LV- & $\begin{array}{c}\text { Concentrated } \\
\text { by }\end{array}$ & $\begin{array}{c}\text { Titer } \\
\text { Supernatant } \\
{\text { [t.u. } / \mathrm{ml}]^{\mathrm{a}}}^{\mathrm{a}}\end{array}$ & $\begin{array}{c}\text { Volume before } \\
\text { concentration } \\
{[\mathrm{ml}]}\end{array}$ & $\begin{array}{c}\text { Volume after } \\
\text { concentration } \\
{[\mathrm{ml}]}\end{array}$ & $\begin{array}{c}\text { Titer after } \\
\text { concentration } \\
{[\text { t.u./ml] }}\end{array}$ & $\begin{array}{c}\text { Recovery } \\
{[\%]}\end{array}$ \\
\hline \multirow{3}{*}{$\begin{array}{l}\text { TPMV- } \\
\text { aCD20 }\end{array}$} & $\begin{array}{c}\text { Low speed } \\
\text { centrifugation }\end{array}$ & $6.9 \times 10^{3}$ & 15 & 0.12 & $4.2 \times 10^{5}$ & 48.5 \\
\hline & $\begin{array}{c}\text { Ultra } \\
\text { centrifugation }\end{array}$ & $4.0 \times 10^{3}$ & 15 & 0.12 & $3.3 \times 10^{4}$ & 6.6 \\
\hline & Ultra filtration & $1.1 \times 10^{4}$ & 15 & 0.24 & $3.7 \times 10^{4}$ & 5.4 \\
\hline \multirow{3}{*}{$\begin{array}{l}\text { MV- } \\
\text { aCD20 }\end{array}$} & $\begin{array}{l}\text { Low speed } \\
\text { centrifugation }\end{array}$ & $4.2 \times 10^{3}$ & 15 & 0.12 & $4.3 \times 10^{5}$ & 82.0 \\
\hline & $\begin{array}{c}\text { Ultra } \\
\text { centrifugation }\end{array}$ & $5.1 \times 10^{3}$ & 15 & 0.12 & $2.5 \times 10^{5}$ & 39.2 \\
\hline & Ultra filtration & $2.4 \times 10^{4}$ & 15 & 0.24 & $1.1 \times 10^{6}$ & 73.5 \\
\hline
\end{tabular}

${ }^{\mathrm{a}}$ Titer in titration units/ml [t.u. $\left./ \mathrm{ml}\right]$

Taken together, of the three tested concentration methods low speed centrifugation seemed to be best suited for the TPMV-pseudotyped vectors, although the recovery was only about $50 \%$. Ultra centrifugation resulted probably in damage of the 
particles since there was only low transduction efficiency after titration of the concentrated vectors. Thus, concentration by ultra filtration was unsuitable for LVTPMVaCD20. Since there was no transduction efficiency observed for the flow through (data not shown), the particles seemed to attach at the membrane of the filter or were damaged. For LV-MVaCD20, highest recovery was achieved by low speed centrifugation, too, but even for UC, there was a much higher recovery than for LV-TPMVaCD20. Concentrating by ultra filtration resulted in high transduction efficiencies for the MV-pseudotypes, indicating that the vectors are still functional and retained by the membrane filter. As described for LV-TPMVaCD20, there was also no transduction observed for MV-pseudotyped vectors by titration of the flow through (data not shown).

\subsubsection{Storage of vector particles}

As mentioned before, for some experiments it is necessary to produce high amounts of vector particles and to store them until needed. It is also important to know the titers of the used vector stocks for some transduction experiments. In general, it takes around 72 hours to observe gene expression and to determine titers after titration of concentrated vectors.

In this experiment it was investigated, if the particles could be stored in the fridge at $4^{\circ} \mathrm{C}$ for 72 hours until the titer of the appropriate vector stock is determined, so that they could be used at the same day for further experiments. Since there is the assumption that the functionality of the vectors decreases when they are frozen and thawed, storage at $4^{\circ} \mathrm{C}$ for 72 hours might be an alternative.

To investigate if there is a loss of function when vectors are frozen and thawed or stored at $4^{\circ} \mathrm{C}$ for 72 hours, TPMV- and MV-pseudotyped particles were produced as described before and concentrated by low speed centrifugation. Afterwards, the pellet was resuspended in $90 \mu \mathrm{l}$ RPMI (+ Glutamine) of which $30 \mu \mathrm{l}$ were used immediately for transduction, $30 \mu \mathrm{l}$ were frozen at $-80^{\circ} \mathrm{C}$ for at least two hours and 30 $\mu l$ were stored in a fridge for 72 hours $\left(4^{\circ} \mathrm{C}\right)$ and subsequent transduction.

Freezing and thawing of LV-TPMVaCD20 resulted in a decrease of titers of $50 \%$ (Fig. 33). After storage of viral particles for 72 hours at $4^{\circ} \mathrm{C}$, almost no transduction efficiency was observed ( $<5 \%$ ), suggesting that the TPMV-pseudotypes lost their functionality. For LV-MVaCD20, there was a decrease of relative titer of only $2 \%$ after freezing and thawing of the vector stock and after storage at $4^{\circ} \mathrm{C}$ for 72 hours, 
still $25 \%$ transduction efficiency could be achieved. Compared to TPMVpseudotypes, LV-MVaCD20 also lost some functionality, but was not sensitive towards freezing and still retained functional after a three-day-storage at $4^{\circ} \mathrm{C}$.

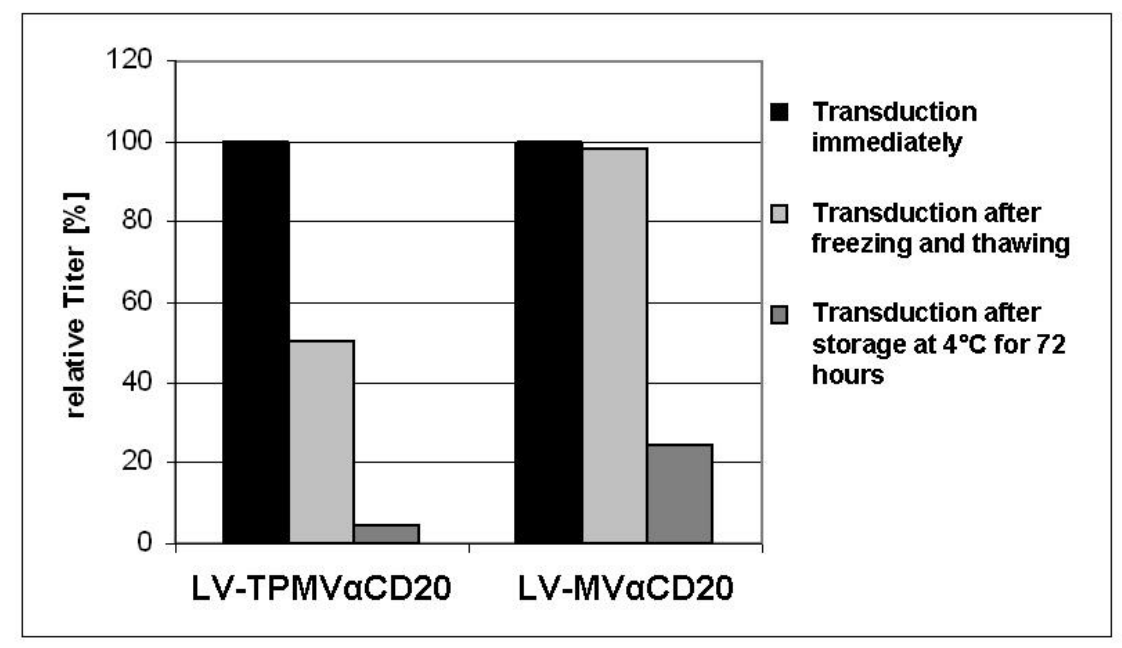

Figure 33: Loss of function after freezing and storage at $4^{\circ} \mathrm{C}$ of TPMV-pseudotyped particles Concentrated vector particles were frozen at $-80^{\circ} \mathrm{C}$ or stored at $4^{\circ} \mathrm{C}$ for 72 hours and afterwards used for transduction of Raji cells. Titers were normalized to that obtained after transduction immediately after concentration by low-speed cetrifugation (100\%).

Up to now all concentrated vector types were resuspended in $\mathrm{RPMI}^{+}$Glutamine . Now that a titer decrease was observed for LV-TPMVaCD20 after freezing and thawing and after storage at $4^{\circ} \mathrm{C}$ for $72 \mathrm{~h}$, the cell culture medium OptiMEM was used as an alternative to $\mathrm{RPMI}^{+}$Glutamine to resuspend the concentrated vector particles. This medium is buffered with HEPES and sodium bicarbonate and supplemented with hypoxanthine, thymidine, sodium pyruvate, L-glutamine, trace elements and growth factors (Invitrogen, complete formulation is confidential). For this experiment the vectors were generated as described before, but after concentration by low speed centrifugation they were resuspended either in RPMI ${ }^{+}$Glutamine , OptiMEM ${ }^{\text {without additives }}$ or OptiMEM supplemented with $1 \%$ bovine serum albumin (BSA), which is often used as a cryoprotective agent.

As already shown before, again there was a decrease of titers for LV-TPMVaCD20 (60\%) when the vectors were frozen and thawed and resuspended in RPMI after concentration (Fig. 34). Using OptiMEM without any additives resulted in a decrease of titers of $40 \%$ for TPMV-pseudotypes, which was also observed for OptiMEM ${ }^{+1 \% B S A}$, suggesting that OptiMEM retained functionality of vector particles but BSA had no additional cryoprotective effect. For LV-MVaCD20, there was an 
increase of titers after freezing and thawing of the vectors (18 - $35 \%$ ), independently, which medium was used to resuspend the vectors after concentration, assuming, that a freeze cycle has an enhancing effect of transduction.

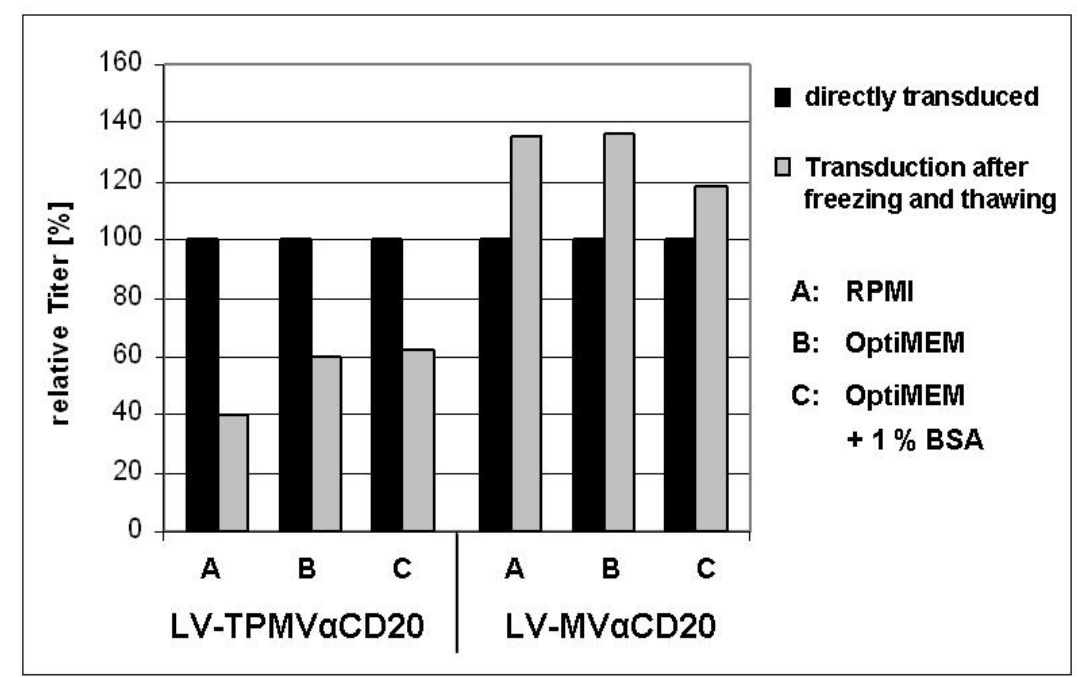

Figure 34: Loss of function of LV-TPMVaCD20 can partially be rescued after freezing in OptiMEM

Concentrated vector particles were resuspended either in RPMI, OptiMEM or OptiMEM supplemented with $1 \%$ BSA (bovine serum albumin) and frozen for at least one hour at $-80^{\circ} \mathrm{C}$. Afterwards, vectors were thawed on ice and used for transduction of Raji cells. Titers were normalized to that obtained after transduction immediately after concentration by low-speed centrifugation (100\%).

In conclusion, it was demonstrated that the highest recovery for TPMVaCD20- and MVaCD20-vector particles could be achieved by low speed centrifugation. However, the recovery was much less for LV-TPMVaCD20 compared to LV-MVaCD20. Freezing and thawing resulted in a third and storage at $4^{\circ} \mathrm{C}$ for three days in a nearly complete loss of functionality for TPMV-pseudotypes, which was not observed for LV-MVaCD20. Resuspending the vector pellet in OptiMEM after concentration had a cryoprotective effect, although there was still a loss of titers of $40 \%$. These data demonstrate that TPMV-pseudotypes are not as stable as MV-pseudotyped vectors based on these performed concentration techniques and storage conditions. For further experiments TPMV- and MV-pseudotyped vectors were concentrated by low speed centrifugation for 24 hours at $4^{\circ} \mathrm{C}$ and the vector pellet was resuspended in OptiMEM without additives. 


\subsection{TPMV-pseudotyped vectors selectively transduce CD20- positive cells}

Having shown that retargeted TPMV-pseudotyped lentiviral vectors could be generated to high titers and identified optimal production conditions, the next step was to test the targeting capability of the vectors. Main objective of gene therapy is the precise transgene expression in selected target cells, so that other cells or tissues remain unaffected. One of the aims of this thesis was to show selective transduction of CD20-positive cells.

\subsubsection{Selective transduction of CD20-positive cells}

For investigation of the selectivity of the retargeted TPMV-pseudotyped vectors, the CD20-positive cell line Raji was mixed in a ratio of 1:1 with CD20-negative myeloid cells (K-562) and incubated with different pseudotyped vectors at a multiplicity of infection (MOI) of 0.3. Vectors were generated as described above. Seventy-two hours post transduction, CD20 positive cells were stained with a PECy5-coupled antibody against CD20 and the amount of GFP-expressing cells in this population was analyzed by FACS.

At a $\mathrm{MOI}$ of 0.3 , transduction of the mixed cell population with LV-TPMVaCD20 resulted in $15.7 \%$ GFP/CD20 double-positive cells, while CD20 negative cells remained almost unaffected (Fig. 35, left column, middle panel). There was virtually no transduction with untargeted TPMV vectors, confirming the assumption that there is no TPMV receptor on human cells (Fig. 35, right column, middle panel). 


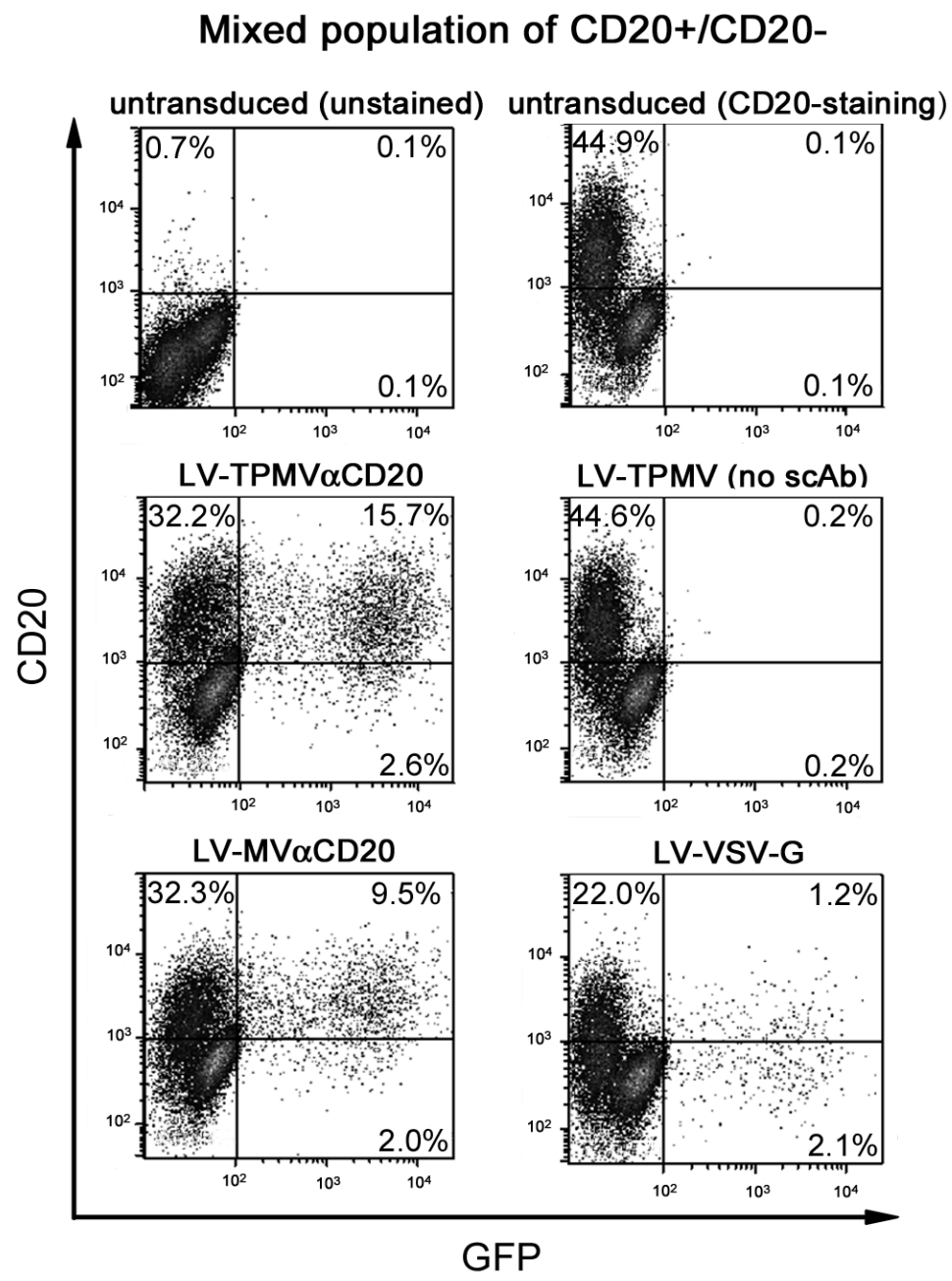

Figure 35: Selective transduction of $\mathrm{CD}^{+} \mathrm{O}^{+}$cells in a mixed cell population

CD20-positive (Raji) and negative (K-562) cells were mixed (1:1) and transduced with different vector types (multiplicity of infection 0.3 ). 72 hours after transduction, the mixed population was stained against CD20 and GFP expression was analyzed by FACS. Untargeted TPMV-pseudotyped vectors were used as a negative control (LV-TPMV). GFP: green fluorescent protein; VSV-G: vesicular stomatitis virus $\mathrm{G}$ protein

Transduction efficiency of the LV-TPMVaCD20 was higher than that of MVpseudotyped vectors that were used as a positive control (9.5\% GFP/CD20 doublepositive cells) (Fig. 35, left column, lowest panel). VSV-G-pseudotyped particles transduced both cell types, although with very low transduction efficiency (Fig. 35, right column, lowest panel), assuming a mistake in calculating the $\mathrm{MOI}$. 


\subsection{Transduction of primary human B cells}

The previous experiments demonstrated that TPMVaCD20-pseudotyped vectors efficiently mediate gene transfer to different CD20-positive cells and transduce only target cells in a mixed cell population. The next step was to investigate whether the vectors are able to enter primary human B cells. These cells naturally express CD20, so the TPMVaCD20-pseudotyped vectors are a promising tool for gene therapy approaches for diseases related to this cell type, like inherited immunodeficiencies, cancer and autoimmune diseases (Frecha et al., 2010).

\subsubsection{Transduction of activated primary human B cells}

Up to date, gene transfer to primary human B cells by viral vectors was not very successful. Lentiviral vectors pseudotyped with the vesicular stomatitis virus $G$ protein (VSV-G) do enter the cells but only with low efficiency (Serafini, Naldini, and Introna, 2004). Recently, it was shown that lentiviral vectors pseudotyped with the MV glycoproteins and targeted to CD20 are able to efficiently transduce primary B cells.

To test whether the novel TPMV vectors can also transduce this cell type, primary human $B$ cells were isolated by negative depletion from peripheral blood mononuclear cells (PBMCs) and activated with a cytokine cocktail for 48 hours. The cocktail consisted of $50 \mathrm{ng} / \mathrm{ml} \mathrm{IL-2,} 10 \mathrm{ng} / \mathrm{ml} \mathrm{IL-4,} 10 \mathrm{ng} / \mathrm{ml} \mathrm{IL-10}$ and $300 \mathrm{ng} / \mathrm{ml}$ CD40-ligand. Incubation of primary B cells with these cytokines should result in expression of the activation marker CD69. To investigate whether CD69 could be detected on the cell surface, the $B$ cells were stained with a fluorescence-coupled antibody and analyzed by FACS.

Stimulation of primary human B cells with the cytokine cocktail resulted in $58.3 \%$ CD69-positive cells that is equivalent to $58.3 \%$ activated cells (Fig. 36). This number is less than expected, but since there is a clear shift of the population compared to the isotype control it was assumed that almost all of the cells were activated and that the low percentage number is due to the high backdround of the isotype control. Control experiments with a different antibody showed an activation rate of about 90 $\%$ (data not shown). 

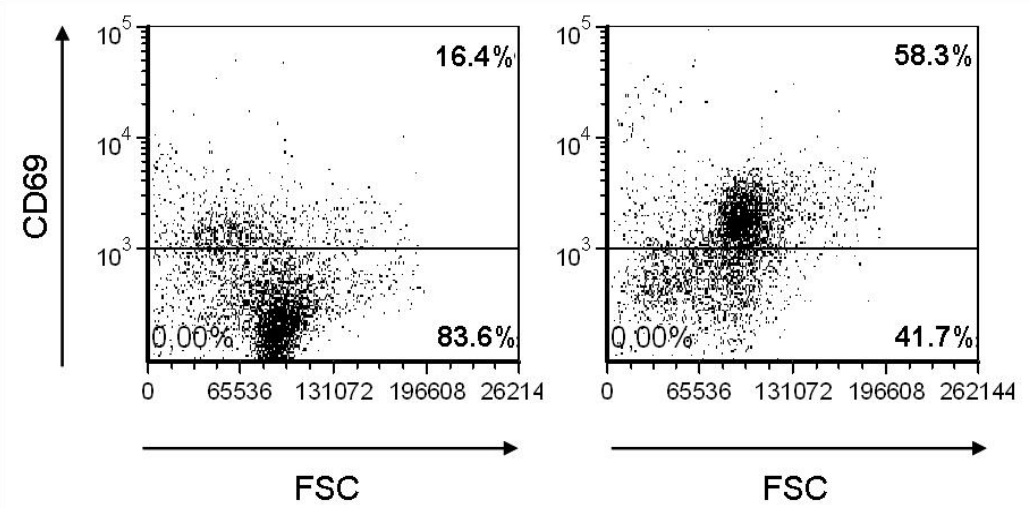

Figure 36: Activated primary human B cells express CD69

Primary human B cells were isolated and immediately activated for 48 hours with a cytokine cocktail containing $50 \mathrm{ng} / \mathrm{ml} \mathrm{IL-2,} 10 \mathrm{ng} / \mathrm{ml} \mathrm{IL-4,} 10 \mathrm{ng} / \mathrm{ml} \mathrm{IL-10}$ and $300 \mathrm{ng} / \mathrm{ml} \mathrm{CD40-ligand.} \mathrm{Expression} \mathrm{of} \mathrm{the}$ activation marker CD69 was then analyzed by FACS. left: B cells incubated with the FITC conjugated $\lg G_{1, \mathrm{~K}}$ isotype control; right: B cells incubated with FITC-conjugated aCD69-antibody

Next, the activated B cells were incubated at an MOl of 1 with concentrated LVTPMVaCD20 and LV- MVaCD20. As mentioned above LV-VSV-G transduce primary human B cells only with low efficiency. In this experiment, concentrated LV-VSV-G was used for comparison at an $\mathrm{MOI}$ of 1 and 100 . Vectors were produced as described before. After 72 hours, cells were stained for the B cell markers CD19 and CD20, and the percentage of GFP/CD19/CD20-triple positive cells was determined by FACS.

The analysis showed that most of the isolated and activated $B$ cells were CD20/CD19-double positive (Fig. 37). Transduction with LV-TPMVaCD20 resulted in 65\% GFP expression of CD19+/CD20+ cells (Fig. 37, A). With LV-MVaCD20, GFP expression was slightly lower (55.4\%, Fig. $37 \mathrm{C})$. As expected, there was only low transduction efficiency with the VSV-G-pseudotyped vectors $(10.0 \%$, Fig. 37, B) even with a $\mathrm{MOI}$ of 100 the transduction efficiency increased only up to $13.2 \%$ (Fig. $37, \mathrm{D})$.

In conclusion, the results showed that it is possible to transduce activated primary human B cells with TPMV-pseudotyped vectors even with better efficiency than with MV-pseudotypes, compared to LV-VSV-G, where transduction efficiency is considerable lower. This result was confirmed with cells from a second donor (data not shown). 
A LV-TPMVaCD20, MOI 1
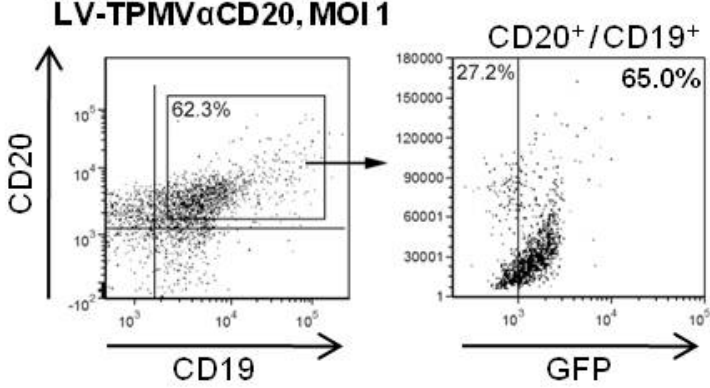

C
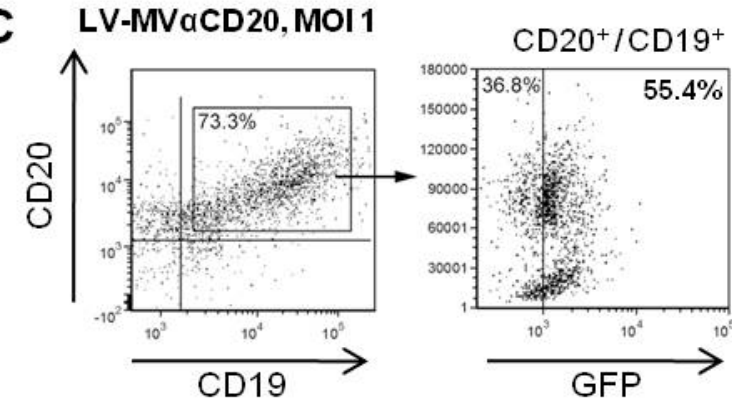

B LV-VSV-G, MOI 1
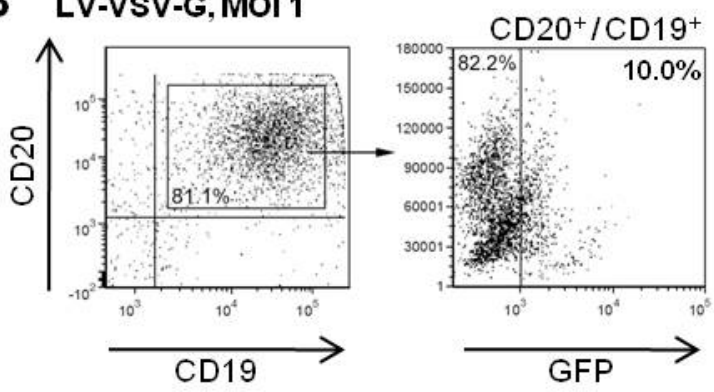

D LV-VsV-G, MOI 100
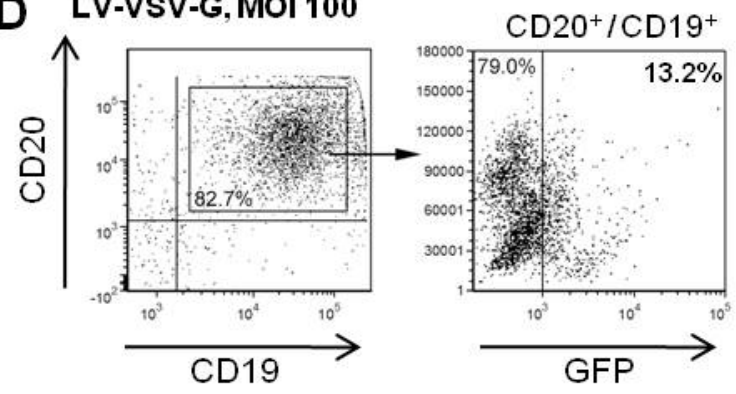

Figure 37: Transduction of activated primary human $B$ cells

Primary human $\mathrm{B}$ cells $\left(\mathrm{CD} 20^{+} / \mathrm{CD} 19^{+}\right)$were isolated from human peripheral blood mononuclear cells and transduced after activation with a cytokine cocktail with LV-TPMVaCD20 (A), LV-VSV-G (B) or LV-MVaCD20 (C) a an MOI of 1. LV-VSV-G was used additionally with a MOI of 100 (D). 48 hours after transduction, cells were stained for the B cell markers CD20 and CD19 and GFP-expression of CD20/CD19 double-positive cells was analyzed by FACS. GFP: green fluorescent protein; VSV-G: vesicular stomatitis virus glycoprotein; MOI: multiplicity of infection

\subsubsection{Transduction of quiescent primary human $B$ cells}

Surprisingly, it was demonstrated, that LV-MVaCD20 was able to efficiently transduce even quiescent primary human B cells (Funke et al., 2008a), a cell type, that was known to be resistant against transduction with lentiviral vectors (Serafini, Naldini, and Introna, 2004). As a next step, it was investigated if LV-TPMVaCD20 is also able to transduce quiescent primary human $B$ cells or if this is due to the MV glycoproteins.

To answer this question, primary human B cells were isolated from human PBMCs by negative depletion and immediately transduced by LV-TPMVaCD20, LVMVaCD20 (MOI 1) and LV-VSV-G (MOI 1 and MOI 100). Before transduction, the cells were stained against the activation marker CD69 to ensure that they were not activated. After 48 hours, the cells were incubated with antibodies against the $B$ cell markers CD19 and CD20, following analysis of GFP expression in this population by FACS.

Antibody-staining of the isolated B cells shows no expression of CD69, confirming that they are not activated (Fig. 38). 

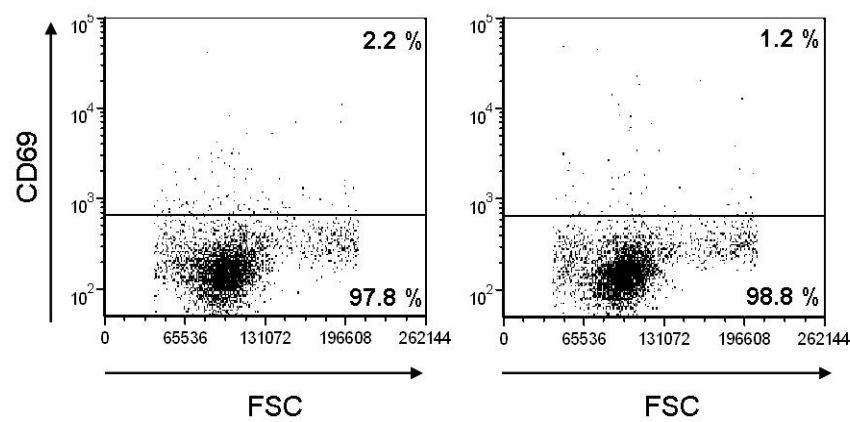

Figure 38: Quiescent primary human B cells do not express the activation marker CD69

Primary human B cells were isolated, immediately stained with an aCD69-antibody and analyzed by FACS. left: B cells incubated with the FITC conjugated $\lg _{1, k}$ isotype control; right: B cells incubated with FITC-conjugated aCD69-antibody

Remarkably, $45.2 \%$ of quiescent B cells were transduced by the TPMV-pseudotyped vector (Fig. 39, A) whereas transduction efficiency for LV-MVaCD20 was slightly less (Fig. 39, C, $35.5 \%$ ). This confirmed the observation already made for activated primary human $B$ cells, namely that LV-TPMVaCD20 transduces this cell type more efficient than MV-pseudotyped vector. As expected, there was virtually no transduction with the VSV-vectors, even not with a MOI of $100(<1 \%$, Fig. 39, B and D).

A LV-TPMVaCD20,MOI 1

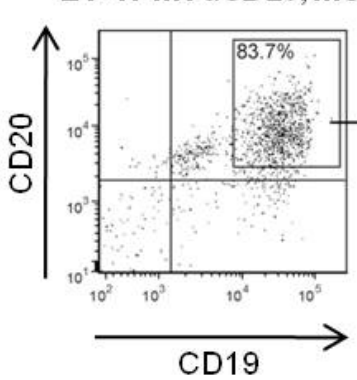

C

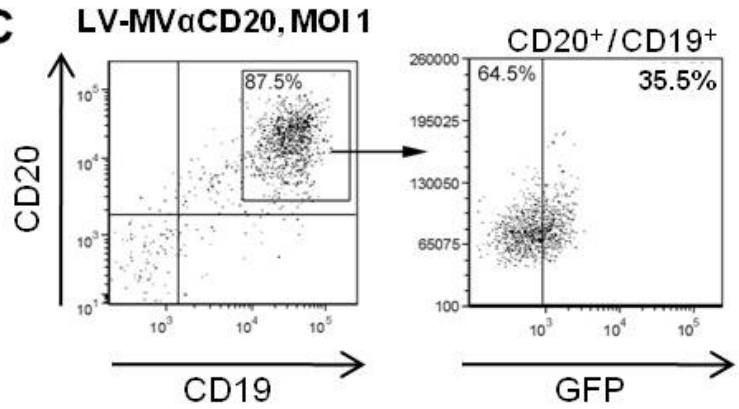

\section{B LV-VsV-G, MOI 1}

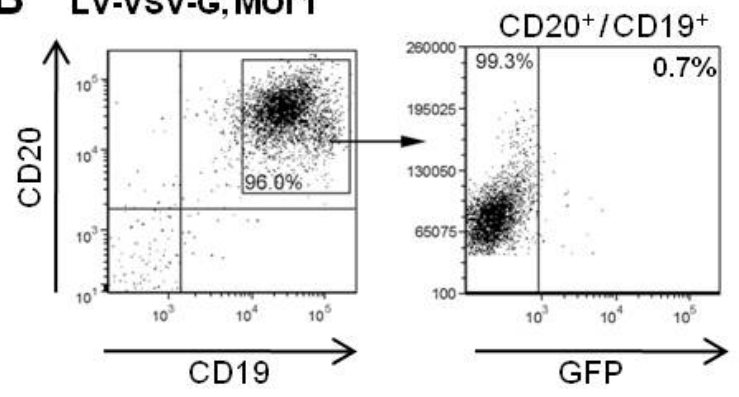

D LV-VSV-G, MOI 100

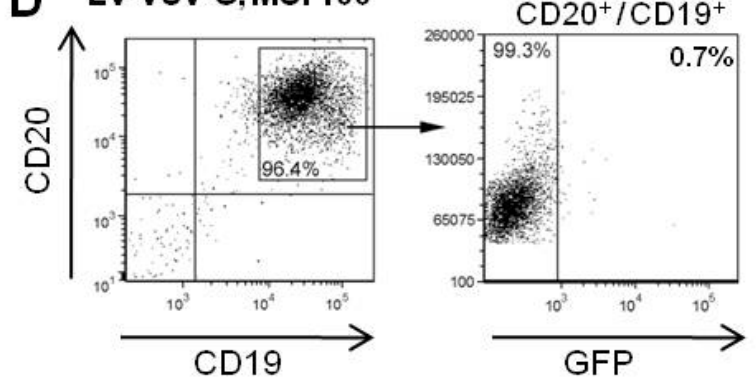

Figure 39: Transduction of quiescent primary human $B$ cells

Primary human $\mathrm{B}$ cells $\left(\mathrm{CD} 20^{+} / \mathrm{CD} 19^{+}\right)$were isolated from human peripheral blood mononuclear cells and transduced immediately with LV-TPMVaCD20 (A), LV-VSV-G (B) or LV-MVaCD20 (C) a an MOI of 1. LV-VSV-G was used additionally with a MOI of 100 (D). 48 hours after transduction, cells were stained for the B cell markers CD20 and CD19 and GFP-expression of CD20/CD19 double-positive cells was analyzed by FACS. GFP: green fluorescent protein; VSV-G: vesicular stomatitis virus glycoprotein; MOI: multiplicity of infection 


\subsubsection{Neutralization assays with retargeted LV-pseudotypes}

Viral vectors have to evade the immune system of the host to reach their target cells. This is problematic for MV-derived vectors for gene therapy because almost everybody in the general human population has measles virus antibodies, either due to $M V$ vaccination or $M V$ infection. At the beginning of this thesis it was hypothesized that TPMV-pseudotyped vectors would not be neutralized by human sera since no antibodies against TPMV are expected in the human population.

To confirm this hypothesis and to test whether TPMV is neutralized or whether there is any cross-reactivity with MV-antibodies, neutralization assays were performed with serum containing MV antibodies, either due to vaccination (serum 1,2) or MV infection (serum 3). As control, MV antibody-negative serum was used (MV-negative serum). The sera were incubated for 30 minutes at $56^{\circ} \mathrm{C}$ to inactivate the complement system. LV-TPMVaCD20, LV-MVaCD20 or vectors pseudotyped with MV glycoproteins that do not contain a scAb (LV-MV(w/o scAb)) were pre-incubated with four different sera in several dilutions and added to CD20-positive Raji cells. After 72 hours, GFP expression of transduced cell was analyzed by FACS. The transduction efficiency of vectors that were incubated with medium only (RPMI) was set as $100 \%$ and vectors incubated with different serum dilutions were normalized to this value.

Transduction efficiency of vectors pseudotyped with MV glycoproteins without ScAb (LV-MV(w/o scAb)) dramatically decreased when vectors incubated with MVantibody-containing sera (sera 1, 2, 3), demonstrating that this vector type was neutralized even with high serum dilutions (Fig. 40). Regarding LV-MVaCD20, this vector type was completely neutralized only by serum 3 , although transduction efficiency also decreased by incubation with serum 1 . Serum 2 had almost no neutralization effect since transduction efficiency was around $80 \%$, even at the lowest serum dilution. These data indicate that the aCD20-scAb had a shielding effect on neutralization of this vector and that higher MV-antibody titers were necessary for neutralization. Transduction efficiency of TPMV-pseudotyped vectors (LV-TPMVaCD20) was decreased after incubation with serum 1, 2 and 3 but did not drop under $35 \%$. No complete neutralization with any of the sera was observed. Remarkably, transduction efficiency of all three vector types (LV-TPMVaCD20, LV$M V a C D 20, L V-M V(w / o ~ s c A b)$ ) was decreased in the presence of serum that does not contain MV antibodies (MV-negative serum). For LV-TPMVaCD20, transduction 
efficiency after incubation with MV-negative serum was similar to that obtained after incubation with serum 3 . This observation might be due to residual activity of the complement system.

In summary, these data show that MV-pseudotypes without scAb were completely neutralized by serum containing MV antibodies. In contrast, neutralization of LVMVaCD20 was less efficient, suggesting a shielding effect of the aCD20-single chain antibody. For this vector type, neutralization seemed to depend on MV-antibody titer of the serum since only with serum 3 a complete neutralization was observed. This serum had probably the highest MV-antibody titer because it was derived from a person that was infected with $\mathrm{MV}$ while serum 1 and 2 were from vaccinated individuals. For TPMV-pseudotypes, no complete neutralization was observed. Although the transduction efficiency decreased, it is not as significant as for the other vector types and since this effect was also observed for MV-negative serum, crossreactivity with MV antibodies could be excluded.
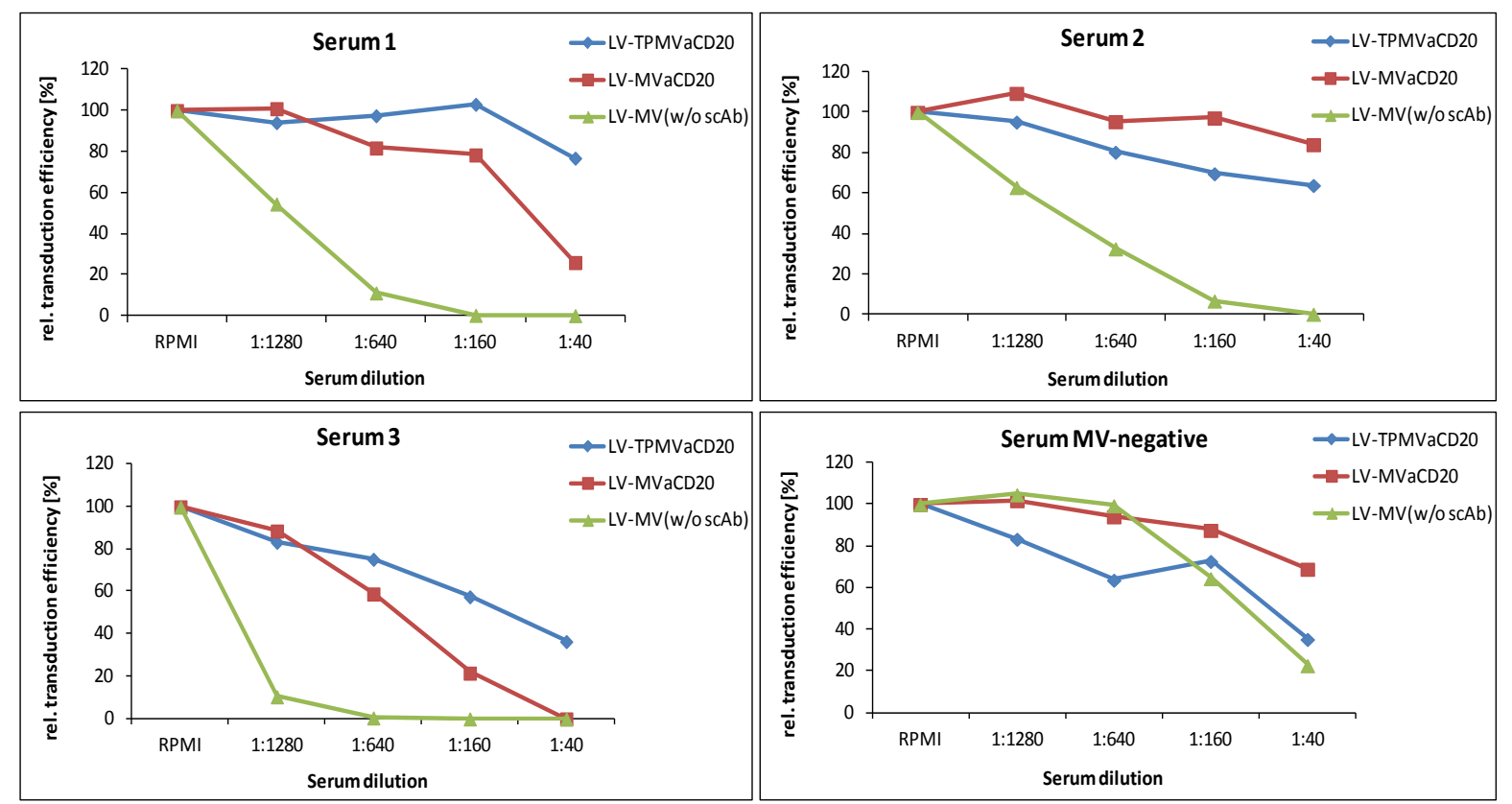

Figure 40: Neutralization assays of pseudotyped LVs with different sera containing MV antibodies

To analyze if MV-antibodies have neutralizing effect on TPMV or show any cross-reactivity, lentiviral vectors pseudotyped with TPMV glycoproteins were incubated with different sera in different dilutions and transduction efficiency was investigated. Vectors pseudotyped with retargeted MV glycoproteins (LV-MVaCD20) or MV glycoproteins without a scAb (LV-MV(w/o scAb)) were used as controls. Serum 1 and 2 were derived from a MV-vaccinated person, serum 3 from a person with MV infection. Serum MV-negative does not contain any MV antibodies. The transduction efficiency of vectors that were incubated with medium only (RPMI) was set as $100 \%$ and vectors incubated with different serum dilutions were normalized to this value. The relative transduction efficiency shown here in percent [\%] is the average of two independent experiments. LV: lentiviral vector; MV: measles virus; TPMV: Tupaia paramyxovirus; scAb: single chain antibody 


\section{DISCUSSION}

Gene therapy is defined as the treatment of a disease or correction of a genetic disorder by introducing therapeutic genes into target cells, respectively (Escors and Breckpot, 2010). To treat a disease or correct a genetic dysfunction, the delivery of therapeutic genes has to be specific to a certain cell type and gene expression has to be efficient. Lentiviral vectors provide a promising tool for gene therapy, since they allow transduction of dividing and non-dividing cells and mediate long-term gene expression by integration of the transgene into the target cell's genome (Matrai, Chuah, and VandenDriessche, 2010). To increase specificity of gene delivery, different approaches have been developed, for example the use of tissue specific promoters so that the gene delivered by the vector is only expressed in target cells (transcriptional targeting) (Waehler, Russell, and Curiel, 2007). However, this method also affects non-target tissues since vectors can enter cells in a non-specific way. Therefore, an important point is to restrict cell entry of vectors to the desired cell type, a process called transductional targeting. This is the most desirable approach since it leaves other tissues or cells completely unaffected (Waehler, Russell, and Curiel, 2007). Although there has been great success in the development of lentiviral vector technology, safety and efficiency of gene transfer have to be further improved (Bouard, Alazard-Dany, and Cosset, 2009; Waehler, Russell, and Curiel, 2007).

Significant progress has been made in the development of MV-based vectors, but they are obviously unsuitable for long-term replacement gene therapy since infected cells are killed and there is no integration of the transgene into the genomes of the infected cells. MV can be targeted to many different cell types by displaying a variety of specificity domains on the $\mathrm{H}$ protein (Cattaneo, 2010; Schneider et al., 2000). Recently, it has been shown that the MV glyoproteins can be used to pseudotype lentiviral vectors (Anliker et al., 2010; Frecha et al., 2009; Frecha et al., 2008; Funke et al., 2008a; Funke et al., 2009). These vectors combine the versatile MV targeting system with the advantages of lentiviral vectors (reviewed in (Buchholz, Mühlebach, and Cichutek, 2009)). But since MV antibodies are widespread in the general population, either due to vaccination or infection with MV, neutralization of these vectors might be an important problem to overcome.

Therefore, in this thesis vectors pseudotyped with glycoproteins of an alternative paramyxovirus were developed, namely the glycoproteins of the Tupaia 
paramyxovirus (TPMV). They can be retargeted in a similar way to MV glycoproteins and since TPMV is an animal paramyxovirus that does not infect humans, no preexisting neutralizing antibodies are expected (Springfeld et al., 2005; Tidona et al., 1999). Furthermore, while the MV glycoproteins have to be modified to ablate receptor binding of the $\mathrm{H}$ protein to natural MV receptors (Nakamura et al., 2005; Vongpunsawad et al., 2004), this is not necessary for the TPMV H protein, resulting in increased safety of these vectors. Hence, in this study, retargeted lentiviral vectors were generated that combine the advantages of MV-pseudotyped vectors with the advantages of TPMV glycoproteins.

\subsection{Characterization of TPMV glycoproteins}

The first step in the development of TPMV-pseudotyped lentiviral vectors was the generation and characterization of modified TPMV $\mathrm{F}$ and $\mathrm{H}$ glycoproteins. Following the observation that unmodified MV glycoproteins are not incorporated into LVs, eight TPMV $\mathrm{H}$ variants and seven TPMV $\mathrm{F}$ variants with different truncations of their cytoplasmic tails were generated. Additionally, a single chain antibody (scAb) against $\mathrm{CD} 20$ was displayed on all truncated $\mathrm{H}$ variants. Western Blot analysis showed that all modified proteins are expressed at levels similar to wild type.

The TPMV glycoproteins are related to the MV glycoproteins but have some uncommon properties. The cytoplasmic tail of the $\mathrm{H}$ protein is considerably longer than those of all other paramyxoviruses. Previous results showed that targeting of the TPMV glycoproteins with a CEA scAb in analogy to MV is possible (Springfeld et al., 2005). In this thesis, a scAb against CD20 was displayed at the ectodomain of TPMV $\mathrm{H}$. Analysis of surface expression of the $\mathrm{H}$ protein variants revealed that the scAb does not inhibit protein processing within the cell. To test the proteins in regard to their function, fusion assays were performed. All truncated TPMV HaCD20 variants were functional. Remarkably, a truncation of 80 or 84 amino acids (aa), leaving only 14 or 10 aa, respectively, is well tolerated in terms of function. For truncated MV H protein, at least 14 aa are necessary to mediate fusion. Variants with shorter CTs lost their functionality (Moll, Klenk, and Maisner, 2002). Demonstrating that the aCD20scAb on TPMV H can also be used for targeting, strengthens the evidence that the TPMV targeting system is as versatile as the MV system. 
In contrast to TPMV $\mathrm{H}$, truncation of the $\mathrm{CT}$ of the $\mathrm{F}$ protein by 34 or more amino acids is not tolerated. As demonstrated in fusion assays, $F$ variants with a cytoplasmic tail of 3 or 4 amino acids lost their function since they are not able to mediate the formation of syncytia. Surprisingly, truncation of 33 amino acids did not affect syncytia formation at all, so a difference of only one amino acid determined functionality. This is in contrast to the truncated MV F protein where only three amino acids of the CT are sufficient to mediate fusion (Moll, Klenk, and Maisner, 2002).

Regarding the details of the TPMV $F$ protein, the F1/F2 cleavage site is different compared to other paramyxovirus fusion proteins and activated by a yet unidentified protease (Springfeld et al., 2005). In general, the F1/F2 cleavage site of paramyxoviruses is preceded either by one or several arginines and lysines, respectively, it is mono- or oligobasic (Klenk and Garten, 1994). Oligobasic cleavage sites are recognized by furin-like proteases in the medial- and trans-Golgi, monobasic cleavage sites by trypsin-like proteases on the cell surface. Exceptions are Nipah (NiV) and Hendra (HeV) viruses, which have amonobasic cleavage site but are neither cleaved by trypsin nor furin. The FO protein of $\mathrm{NiV}$ and $\mathrm{HeV}$ is transported to the cell surface and subsequently endocytosed. Afterwards, it is cleaved in the endosome by cathepsin $\mathrm{L}$ into activated $\mathrm{F} 1$ and $\mathrm{F} 2$ and transported back to the cell surface (Lee and Ataman, 2011). TPMV has neither a mono- nor an oligobasic cleavage site.

For characterization of the TPMV F protein, a new antibody $\left(\mathrm{aF}_{\text {ecto }}\right)$ was generated against a peptide located in the proteins ectodomain. Since the antibody that was previously used to characterize TPMV $\mathrm{F}$ was directed against the cytoplasmic tail $\left(\mathrm{aF}_{\mathrm{cyt}}\right)$, this antibody could not be used to analyze the cytoplasmic tail-deleted proteins. With the new antibody $\alpha F_{\text {ecto, }}$, a novel fragment was detected, suggesting that a significant fraction of the F1 protein is further cleaved into an F1a and F1b fragment, similar to the MV and CDV F protein (von Messling et al., 2004). F proteins of Morbiliviruses are contranslationally inserted into the membrane of the endoplasmatic reticulum (ER), glycosylated and folded with subsequent trimer formation (Hernandez et al., 1996). These $F$ protein trimers are arranged into a ringlike structure to form the fusion pore. For MV and CDV $F$ proteins it was hypothesized that partial $\mathrm{F} 1 \mathrm{a} / \mathrm{F} 1 \mathrm{~b}$ cleavage induces a conformational change of the trimers that leads to fusion pore formation by breaking symmetry. 
To further characterize the newly observed TPMV F1a/F1b cleavage a number of experiments were performed. The data revealed that the novel F1a fragment is transported along the secretory pathway and secreted into the supernatant of transfected cells. Furthermore, significant amounts were also detected in purified TPMV virions. The cleavage site was mapped and surprisingly, it is located within the outer half of the transmembrane domain of TPMV F. These results indicate that F1a is no longer anchored in the cell membrane and can therefore be released into the supernatant. The presence of $\mathrm{F} 1 \mathrm{a}$ in virions can probably be explained by the formation of mixed trimer complexes that contain membrane-anchored F1 as well as F1a.

The cleavage site was mapped by insertion of a Flag-tag at different amino acid positions in the ectodomain expecting that $\mathrm{F} 1 \mathrm{a} / \mathrm{F} 1 \mathrm{~b}$ cleavage results in loss of protein detection (section 6.3.1). With an aFlag-antibody, the $F 0$ fragment of $F_{499}$ could be detected but not of $F_{500}$, indicating that the cleavage site is located between amino acid position 499 and 500 . Surprisingly, no $F 1$ fragment was found for $F_{483}-F_{499}$ using the aFlag-antibody, but when the Flag-tag was added after aa position 533 or at the $C$-terminal end of the $F$ protein $\left(F_{553}\right)$, the $F 1$ fragment was visible.

In the case of the MV and CDV F protein, it was not possible to completely ablate $F$ protein function by mutating the $\mathrm{F} 1 \mathrm{a} / \mathrm{F} 1 \mathrm{~b}$ cleavage site. Mutation or substitution of the respective amino acid sequence only reduced cleavage efficiency. These data demonstrate that factors other than the primary amino acid sequence, for example an exposed structure, influence protease cleavage (von Messling et al., 2004). Similar results were obtained by mutating the $\mathrm{F} 1 \mathrm{a} / \mathrm{F} 1 \mathrm{~b}$ cleavage site of TPMV $\mathrm{F}$ (section 6.3.2). The respective amino acids were replaced by alanine residues. Replacement of the amino acids of the cleavage site by alanins did not result in cleavage inhibition. Characterization of the cleavage protease using the inhibitors Antipain, E-64 and Leupeptin (section 6.4.1) revealed that both $F$ protein cleavages (F1/F2 and $\mathrm{F} 1 \mathrm{a} / \mathrm{F} 1 \mathrm{~b}$ ) did not occur, resulting in an inactive $\mathrm{F}$ protein as demonstrated in fusion assays. These three chemicals have in common that they are cysteine protease inhibitors, suggesting that a cysteine protease is involved in cleavage and subsequent activation of TPMV F. Antipain and leupeptin are peptidyl aldehydes and their inhibition is reversible. E-64 is an irreversible inhibitor and belongs to the group of epoxysuccinyl-based inhibitors which only inhibit cysteine proteases. All three substances contain a peptide segment for recognition by the enzyme which is 
coupled to a nucleophilic group. This group can attack or substitute the cysteine residue of the active site of the enzyme (Otto and Schirmeister, 1997).

Interestingly, for $\mathrm{NiV}$ and $\mathrm{HeV}$, the endosomal/lysosomal cysteine protease cathepsin $L$ has been identified as an activating protease (Craft and Dutch, 2005; Meulendyke et al., 2005; Pager et al., 2006; Pager and Dutch, 2005). As mentioned before, NiV F0 is cleaved in the endosome by $L$ cathepsin forming F1 and F2 after clathrinmediated endocytosis. This is due to a tyrosine-based internalization signal in its cytoplasmic tail, the YSRL sequence (aa 525-528) (Diederich et al., 2005). Similar results were obtained for $\mathrm{HeV}$ (Pager et al., 2006; Pager and Dutch, 2005). The YSRL sequence, which is located in the CT of NiV and HeV F protein, fits in the YXXФ-type endocytosis signal consensus motif, whereat $Y$ is a tyrosine, $X$ can be any amino acid (aa) and $\Phi$ is a large hydrophobic aa. Tyrosine-dependent motifs are often involved in protein sorting and targeting signals of transmembrane proteins (Bonifacino and Traub, 2003). Regarding the CT of TPMV F, two tyrosine residues can be found at aa positions 527 and 529. At aa position 529-532 of TPMV $F$ the sequence YSLR is present which is similar to the endocytosis signal of $\mathrm{NiV}$ and $\mathrm{HeV}$. But since it contains an arginine instead of a hydrophobic aa at position four, it does not match the YXXФ-type endocytosis signal. Another tyr-containing motif can be found at aa position 527-530 (YVYS), but this also lacks the hydrophobic aa at position four of the YXXФ- motif. However, there are some exceptions. The Influenza HA protein is known to not be endocytosed, but a cytoplasmic tail mutant carrying a tyrosine at aa position 453 and a serine at aa position 546 (HA-Y543/S546), resulting in the motif YXXS, was endocytosed efficiently (Naim and Roth, 1994). Regarding the CT of TPMV F, the sequence YVYS (aa 527-530) is equivalent to the YXXS motif of the Influenza HA-mutant. Consequentially, it might act as endocytosis signal for TPMV F. Furthermore, it indicates that the consensus motif is possibly variable.

Supporting this hypothesis is the detection of high amounts of F0 fragment in addition to F1 in Western Blot analysis of TPMV-pseudotyped vectors (section 6.5.4). This indicates that only a fraction of $F$ protein was cleaved and activated on the cell surface which might have consequences for the generation of TPMV-pseudotyped LVs. Since glycoprotein expression on the cell surface is a critical step in vector formation, titers might be reduced due to the high amount of uncleaved $F$ protein. In contrast to this observation, there was no F0 detected in TPMV virions (section 6.2.4). Possibly, the virus has a different mechanism of $F$ protein processing that only 
allows incorporation of cleaved protein, for example, assembly in specialized domains of the cell membrane. An alternative explanation is that the truncated variant $F \Delta 32$ is incorporated in $L V s$ in contrast to the full-length $F$ protein in virions assuming that an endocytosis signal sequence in the CT was removed by truncation. Thus, endocytosis and subsequent cleavage and activation of F $\Delta 32$ might not have been complete.

The experiments performed in this section revealed only preliminary results, for a better understanding of the mechanism behind TPMV $F$ cleavage and activation and to verify the above described hypothesis, further investigation is necessary. To find out whether $\mathrm{F}$ cleavage occurs during transport along the secretory pathway or at the plasma membrane, protein expression of cells treated with inhibitors of the secretory pathway has to be analyzed. For analysis of the endocytosis hypothesis, the respective tyrosine residues of the TPMV F cytoplasmic tail could be mutated and endocytosis of the native and mutant protein could be investigated in presence or absence of endocytosis inhibitors. Characterization of the protease revealed that a cysteine protease is likely to be involved in cleavage. The most important cysteine proteases in mammals are calpains which can be found in the cytoplasm and cathepsins that are mainly located in the lysosomes (Otto and Schirmeister, 1997), but it has been shown that cathepsins can also be secreted when cells are stimulated with proinflammatory cytokines (Reddy, Zhang, and Weiss, 1995; Sukhova et al., 1998; Turk, Turk, and Turk, 2000). Other important members of the cysteine protease family are caspases which are involved in apoptosis (Amarante-Mendes and Green, 1999). Since it was shown that the $F 1 a / 1 b$ cleavage site is located in the transmembrane domain, the responsible protease could also be an extracellular protease. To further restrict the cysteine protease that is responsible for TPMV $F$ cleavage and activation, investigation of protein expression in the presence of calpain inhibitors (calpeptin, PD150606), caspase inhibitors (caspase inhibitor III) or cathepsin inhibitors (Cath I, Cath LIII) has to be examined.

Identification of the cleavage protease might be important since it has been reported that it can act as target for viral therapy. For NiV and $\mathrm{HeV}$ it has been shown that treatment of virus-infected cells with chloroquine prevents virus spread in vitro (Freiberg et al., 2010). Unfortunately, this effect could not be observed in a golden hamster model in vivo. It has been proposed that chloroquine prevents the proteolytic cleavage of Henipavirus F protein by cathepsin L (Porotto et al., 2009). Although 
TPMV does not infect humans and therefore no treatment is necessary, the virus might be used as a model to further investigate the characteristics of the emerging and highly pathogenic Henipaviruses.

The data raise the question of the biological function of the partial F1a/F1b cleavage. As mentioned before, one hypothesis is that proteolytic processing enhances fusion pore formation (von Messling et al., 2004). Another function of this cleavage could be that a fragment of TPMV $F$ acts as modulator of the host's antiviral immune response. Importantly, there is an example from a paramyxovirus $F$ protein, the bovine respiratory syncytial virus (BRSV). Cleavage of the fusion protein results in a small fragment that is converted into a biologically active molecule by additional posttranslational modifications (Zimmer, Rohn et al. 2003). This molecule is called virokinin and can interact with tachikinin receptors of the host that are involved in local inflammatory and immune processes.

Although there are still open questions about the mechanism of $F$ protein activation, the TPMV glycoproteins share many features with MV glycoproteins that make them so suitable for pseudotyping of lentiviral vectors.

\subsection{Pseudotyping of lentiviral vectors with TPMV glycoproteins}

After characterization of TPMV glycoprotein variants they were screened in all combinations for their ability to pseudotype lentiviral vectors. Screening revealed that the $\mathrm{F}$ protein variant with a 6-amino acid tail $(\mathrm{F} \Delta 32)$ together with the $\mathrm{H}$ variant with a tail length of 14 aa $(\mathrm{H} \Delta 80 \alpha \mathrm{CD} 20)$ resulted in the highest vector titers (section 6.5.2). As already mentioned for the fusion assays, truncations of the long cytoplasmic tail of TPMV $\mathrm{H}$ were surprisingly well tolerated. Although the fusogenicity of the protein was reduced when the cytoplasmic tail was truncated, there was no complete loss of function when a certain tail length was reached. In contrast to TPMV $\mathrm{H}$, no functional vectors could be generated with TPMV F proteins with truncations of more than 33 amino acids. Interestingly, transduction was achieved with $\mathrm{F} \Delta 33$ but not with $\mathrm{F} \Delta 34$ or F $\Delta 35$. Apparently, a difference of only one amino acid prevents functional LVpseudotyping. This could be due to a loss of function of these proteins or to low or even no incorporation levels into LVs. But as fusion assays have demonstrated that $F$ proteins with short CT do not form syncytia, there is probably a loss of function of these $F$ variants. Replacing the CT of TPMV F with that of truncated MV F protein 
also did not lead to a functional protein,. An interesting observation was that even TPMV glycoproteins without any CT truncations were able to pseudotype LVs, although titers were low. This is in contrast to MV-pseudotypes. No vectors could be generated with full-length glycoproteins. Different results were also obtained regarding the length of the CT. For the MV F protein, a truncation of the cytoplasmic tail to three amino acids was found to result in the highest vector titers (Frecha et al., 2008; Funke et al., 2008b). Similar results had been obtained previously with the F protein of Sendai virus that had a cytoplasmic tail truncation to four amino acids (Kobayashi et al., 2003). For the MV H protein, conflicting results have been published: while one group found a truncation to 16 amino acids most effective (Funke et al., 2008b), another group reported a truncation to 10 amino acids to be optimal (Frecha et al., 2008). An explanation for the differences between MV and TPMV could be that the cytoplasmic tails have no sequence similarities. In conclusion, it was not possible to directly transfer the results obtained for $\mathrm{MV}$ to TPMV but CT optimal truncations of both glycoproteins are approximately in the same range. For the $\mathrm{F}$ proteins, keeping the CT as short as possible was most effective to maintain function. Another difference to the MV system is that the attempts to increase vector titers by using different ratios of the TPMV $\mathrm{F}$ and $\mathrm{H}$ plasmids during vector production were not successful. For MV it was reported that a $\mathrm{H}: \mathrm{F}$ plasmid ratio of $1: 7$ increased titers by up to ten-fold compared to the same amounts of $\mathrm{H}$ and $\mathrm{F}$ plasmids. In cells infected naturally with $\mathrm{MV}$, there is a gradient of mRNA due to the MV gene order. Accordingly, more $\mathrm{F}$ than $\mathrm{H}$ mRNA is produced in MV infected cells (Cattaneo et al., 1987b; Plumet, Duprex, and Gerlier, 2005). Thus, the situation in natural MV infected cells is simulated by the use of higher amounts of $\mathrm{F}$ plasmid. However, an increase of the amount of TPMV F plasmid had no effect on vector titer although gene order in the TPMV genome is the same as for MV.

Regarding stability assays performed in this thesis it is obvious that the TPMVpseudotypes are not as stable as MV-pseudotypes. But finally, vector titers similar to those in the MV system were achieved. Compared to lentiviral vectors pseudotyped with VSV, these titers are still low and for using them in gene therapy trials, vector production has to be further improved. To further increase vector titers, a more detailed understanding of the requirements for glycoprotein incorporation into lentiviral particles and of the molecular biology of TPMV will probably be necessary. 
To investigate the specificity of the TPMV-pseudotyped vectors, different cell lines were transduced with different vector types (section 6.5.5). There was virtually no background transduction with TPMV-pseudotypes, demonstrating the absence of a receptor for TPMV and the CD20-mediated specific entry of the vectors. This is in contrast to MV-pseudotypes, where some background transduction could be observed. This might be due to incomplete mutation of the CD46/SLAM contact residues or other receptors, e.g. nectin-4 (Noyce et al., 2011). The MV glycoproteins used as controls in this thesis are so far blinded against this receptor. Specificity of TPMVaCD20-LVs was furthermore demonstrated by transduction of a mixed cell population of CD20-positive and -negative cells (section 6.7). TPMV-pseudotyped vectors selectively entered CD20-positive cells while VSV-G pseudotyped vectors do not discriminate between the two cell lines.

An important point of this thesis was the transduction of primary human $B$ lymphocytes. Previous reports described that lymphocytes require stimulation with cytokines or other factors for transduction with lentiviral vectors (Serafini, Naldini, and Introna, 2004; Unutmaz et al., 1999). Remarkably, transduction of stimulated human B cells with LV-TPMVaCD20 resulted in 65.0 \% GFP-positive cells (section 6.8.1). Regarding LVs pseudotyped with VSV-G, they are able to transduce most target cells, but transduction levels of human B cells even under optimized conditions are low (Bovia et al., 2003; Janssens et al., 2003). In this study when using an MOI of 100 only $13.2 \%$ of the stimulated B cells were GFP-positive after transduction. Hence, the retargeted TPMVaCD20 glycoproteins are significantly more efficient in mediating gene transfer to primary human B cells than the VSV glycoprotein.

Gene transfer into quiescent lymphocytes might be of even more importance for gene therapy approaches than in activated lymphocytes, since they are involved in many diseases, like inherited immunodeficiencies, cancer and autoimmune diseases (Frecha et al., 2010). Quiescent lymphocytes remain in the G0 phase of the cell cycle until they are stimulated. During this time, the cell size is reduced and transcription and translation rates as well as metabolism are down regulated (Buchholz, Mühlebach, and Cichutek, 2009). Viral infection of cells in the G0 phase does not lead to the respective post-entry steps like reverse transcription of the virus genome and transport to the nucleus. Consequently, the transgene is not integrated in the host genome (Korin and Zack, 1998; Yoder et al., 2008). After stimulation, for 
example by cytokines or receptor binding, the cell enters the G1 phase and down regulation is reversed.

Recently, efficient transduction of quiescent primary human lymphocytes with measles virus pseudotyped LVs was reported independently by two different groups (Frecha et al., 2011; Zhou et al., 2011). Unexpectedly, binding to both natural receptors (CD46 and SLAM) of the MV vaccine strain seems to be necessary. However, lentiviruses pseudotyped with a mutated $\mathrm{MV} \mathrm{H}$ protein were unable to mediate entry via CD46 or SLAM, but when targeted with a aCD20-scAb could also be used to transduce quiescent B cells (Funke et al., 2008b). In this thesis it was shown that LVs pseudotyped with TPMV glycoproteins carrying the same aCD20scAb also mediate efficient gene transfer into quiescent primary human B cells (45.2 $\%$ GFP-positive cells) Efficiency was even higher than MVaCD20-LVs that were used with the same $\mathrm{MOI}$ as control (35.5 \% GFP-positive cells) (section 6.8.2). The quiescent lymphocytes were not transduced by VSV-G-pseudotyped LVs, even when a high multiplicity of infection (MOI 100) was used.

For the targeted MV-pseudotyped vectors two mechanisms were proposed (Buchholz, Mühlebach, and Cichutek, 2009) that mediate transduction of quiescent lymphocytes. Regarding the first mechanism it has been speculated that residual binding of the $\mathrm{H}$ protein to CD46 and SLAM might induce changes in the actin rearrangement and microtubule formation of the target cells that make them susceptible for lentiviral transduction (Buchholz, Mühlebach, and Cichutek, 2009). Another hypothesis is that binding of the retargeted MV H protein to CD20 acts as a proliferative stimulus and as a consequence, resting B cells are activated and become susceptible for transduction. The results obtained in this thesis using retargeted TPMV-pseudotyped vectors exclude the first hypothesis as TPMV does not enter cells via the receptors CD46 and SLAM. But the retargeted TPMV H protein carries the same $a C D 20-s c A b$ as the retargeted MV $\mathrm{H}$ protein, confirming that binding of the scAb to CD20 is sufficient to mediate transduction of quiescent primary human B cells. This observation supports the hypothesis that binding to CD20 induces an activation signal, leading to susceptibility towards vector entry.

The natural role of the CD20 molecule is still not fully understood. Although an antiCD20, B-cell-specific monoclonal antibody (Rituximab) has been approved for several years for treatment of immune diseases like Non-Hodgkin's lymphoma and rheumatoid arthritis, the underlying molecular mechanism has not been completely 
resolved. However, further investigation of the CD20 molecule and post-entry steps of pseudotyped lentiviral vectors are necessary to reveal the molecular mechanism of the CD20 molecule.

At the beginning of this thesis it was postulated that the TPMV glycoproteins are similar enough to MV glycoproteins to provide all properties for efficient pseudotyping but different enough to escape neutralization by $M V$ antibodies. To verify this hypothesis neutralization assays with TPMV-pseudotyped vectors were performed (section 6.8.3). Serum containing MV antibodies, either due to vaccination or infection, was used in these experiments. Since the TPMV glycoproteins only have low similarity to MV glycoproteins in their amino acid sequences (TPMV F: $33.4 \%$; TPMV H: < $20 \%$ ), no cross-reactivity was expected. Indeed, compared to the control vector pseudotyped with non-targeted MV glycoproteins, the TPMV-pseudotyped vectors escape neutralization. There is a slight decrease of the relative transduction efficiency for LV-TPMVaCD20 but since this effect was also observed for MVnegative serum with increasing serum concentrations, this might be due to other factors than cross-reactive antibodies, for example the complement system. Interestingly, the retargeted MVaCD20 vectors were not neutralized as much as the non-targeted vectors, suggesting that the scAb displayed on the MV $\mathrm{H}$ protein provides a shielding effect. But with higher antibody concentrations the shielding effect is ablated. This experiment demonstrates that glycoprotein exchange of pseudotyped vectors with glycoproteins of an animal virus provides an escape option from neutralizing antibodies. A similar effect was recently described for a mutant MV where the MV glycoproteins were replaced by the glycoproteins of the animal paramyxovirus Canine distemper virus (CDV). In an animal model it was shown that the therapeutic effect of the envelope-exchanged virus was not affected by MV antibodies (Miest et al., 2011). Now it has to be investigated if TPMV-pseudotyped LVs are also able to escape MV neutralizing antibodies in vivo. 


\subsection{Outlook}

The results obtained in this thesis demonstrate that it is possible to generate lentiviral vectors with paramyxovirus envelope proteins of a non-human virus that will not be neutralized by preexisting antibodies in patients. This is an important advantage towards MV glycoproteins used for pseudotyping. The TPMV glycoproteins possess all properties of MV glycoproteins making them interesting for pseudotyped LVs for gene therapy. They can be retargeted, they are incorporated into LVs when their CTs are truncated and the titers obtained are similar to those obtained with MV glycoproteins. Additionally, TPMV proteins escape neutralization by MV antibodies. This is also the first time, that a LV pseudotyped with other proteins than MV proteins could achieve transduction of quiescent primary human B cells.

One potential disadvantage of these newly developed vectors is their lower stability compared to MV-based LVs. This might be critical when it is necessary for gene therapy to produce large amounts of vectors that can be stored for a longer period. To overcome this problem, possibly further protein modifications are necessary to increase titers and stability. Since TPMV is an animal paramyxovirus, the TPMV glycoproteins probably have to be adapted to the human transcription and translation machinery. This can be done for example by modification of the codon usage. For the generation of Human Papilloma virus (HPV) virus-like particles it was shown that codon optimization increased protein-levels by about 100-fold (Mossadegh et al., 2004). Converting the codons of TPMV glycoproteins to those more common in human genes might increase protein expression and thereby incorporation levels into the viral envelope. Another attempt to increase stability and titers is to exchange the cytoplasmic tails of the TPMV glycoproteins. It has recently been reported that LVs pseudotyped with rabies virus glycoproteins show increased incorporation levels when the rabies CT is replaced by the CT of VSV (Carpentier et al., 2011). Since VSV-G-pseuodtyped LVs are very stable, it might be possible to increase stability of TPMV-pseudotypes by exchanging the cytoplasmic tail of the $F$ protein as it was done with chimeric rabies/VSV-G-pseudotyped vectors.

For the TPMV $\mathrm{H}$ protein it was shown that retargeting with $a C E A s c A b$ and $a C D 20 s c A b$ is possible, suggesting that the TPMV system is as versatile as the MV system. Since most cell types targeted for gene therapy express unique surface antigens against which scAbs can be generated, the results obtained in this thesis 
open the exciting perspective of targeted lentiviral transduction of any target tissue after systemic administration of gene therapy vectors.

TPMV vectors will certainly induce neutralizing antibodies when administered systemically to patients. Since in many cases repeated administration of gene therapy vectors will probably be necessary for efficient transduction of the target tissue, it might be reasonable to further pursue the identification of other animal paramyxovirus envelope proteins that, like TPMV, can be targeted and used to pseudotype lentiviral vectors. 


\section{REFERENCES}

Akkina, R. K., Walton, R. M., Chen, M. L., Li, Q. X., Planelles, V., and Chen, I. S. (1996). High-efficiency gene transfer into CD34+ cells with a human immunodeficiency virus type 1-based retroviral vector pseudotyped with vesicular stomatitis virus envelope glycoprotein G. J Virol 70(4), 2581-5.

Amarante-Mendes, G. P., and Green, D. R. (1999). The regulation of apoptotic cell death. Braz J Med Biol Res 32(9), 1053-61.

Anderson, W. F., Blaese, R. M., and Culver, K. (1990). The ADA human gene therapy clinical protocol: Points to Consider response with clinical protocol, July 6, 1990. Hum Gene Ther 1(3), 331-62.

Anliker, B., Abel, T., Kneissl, S., Hlavaty, J., Caputi, A., Brynza, J., Schneider, I. C., Munch, R. C., Petznek, H., Kontermann, R. E., Koehl, U., Johnston, I. C., Keinanen, K., Muller, U. C., Hohenadl, C., Monyer, H., Cichutek, K., and Buchholz, C. J. (2010). Specific gene transfer to neurons, endothelial cells and hematopoietic progenitors with lentiviral vectors. Nat Methods 7(11), 929-35.

Blaese, R. M., Culver, K. W., Chang, L., Anderson, W. F., Mullen, C., Nienhuis, A., Carter, C., Dunbar, C., Leitman, S., Berger, M., and et al. (1993). Treatment of severe combined immunodeficiency disease (SCID) due to adenosine deaminase deficiency with CD34+ selected autologous peripheral blood cells transduced with a human ADA gene. Amendment to clinical research project, Project 90-C-195, January 10, 1992. Hum Gene Ther 4(4), 521-7.

Bobkova, M., Stitz, J., Engelstadter, M., Cichutek, K., and Buchholz, C. J. (2002). Identification of R-peptides in envelope proteins of C-type retroviruses. J Gen Virol 83(Pt 9), 2241-6.

Bonifacino, J. S., and Traub, L. M. (2003). Signals for sorting of transmembrane proteins to endosomes and lysosomes. Annu Rev Biochem 72, 395-447.

Bouard, D., Alazard-Dany, D., and Cosset, F. L. (2009). Viral vectors: from virology to transgene expression. Br J Pharmacol 157(2), 153-65.

Bovia, F., Salmon, P., Matthes, T., Kvell, K., Nguyen, T. H., Werner-Favre, C., Barnet, M., Nagy, M., Leuba, F., Arrighi, J. F., Piguet, V., Trono, D., and Zubler, R. H. (2003). Efficient transduction of primary human B lymphocytes and nondividing myeloma B cells with HIV-1-derived lentiviral vectors. Blood 101(5), 1727-33.

Bucheit, A. D., Kumar, S., Grote, D. M., Lin, Y., von Messling, V., Cattaneo, R. B., and Fielding, A. K. (2003). An oncolytic measles virus engineered to enter cells through the CD20 antigen. Mol Ther 7(1), 62-72.

Buchholz, C. J., Mühlebach, M. D., and Cichutek, K. (2009). Lentiviral vectors with measles virus glycoproteins - dream team for gene transfer? Trends Biotechnol 27(5), 259-65. 
Bukrinsky, M. I., Haggerty, S., Dempsey, M. P., Sharova, N., Adzhubel, A., Spitz, L., Lewis, P., Goldfarb, D., Emerman, M., and Stevenson, M. (1993). A nuclear localization signal within HIV-1 matrix protein that governs infection of nondividing cells. Nature 365(6447), 666-9.

Carpentier, D. C., Vevis, K., Trabalza, A., Georgiadis, C., Ellison, S. M., Asfahani, R. I., and Mazarakis, N. D. (2011). Enhanced pseudotyping efficiency of HIV-1 lentiviral vectors by a rabies/vesicular stomatitis virus chimeric envelope glycoprotein. Gene Ther.

Cartier, N., Hacein-Bey-Abina, S., Bartholomae, C. C., Veres, G., Schmidt, M., Kutschera, I., Vidaud, M., Abel, U., Dal-Cortivo, L., Caccavelli, L., Mahlaoui, N., Kiermer, V., Mittelstaedt, D., Bellesme, C., Lahlou, N., Lefrere, F., Blanche, S., Audit, M., Payen, E., Leboulch, P., I'Homme, B., Bougneres, P., Von Kalle, C., Fischer, A., Cavazzana-Calvo, M., and Aubourg, P. (2009). Hematopoietic stem cell gene therapy with a lentiviral vector in X-linked adrenoleukodystrophy. Science 326(5954), 818-23.

Cattaneo, R. (2010). Paramyxovirus entry and targeted vectors for cancer therapy. PLoS Pathog 6(6), e1000973.

Cattaneo, R., Miest, T., Shashkova, E. V., and Barry, M. A. (2008). Reprogrammed viruses as cancer therapeutics: targeted, armed and shielded. Nat Rev Microbio/ 6(7), 529-40.

Cattaneo, R., Rebmann, G., Baczko, K., ter Meulen, V., and Billeter, M. A. (1987a). Altered ratios of measles virus transcripts in diseased human brains. Virology 160(2), 523-6.

Cattaneo, R., Rebmann, G., Schmid, A., Baczko, K., ter Meulen, V., and Billeter, M. A. (1987b). Altered transcription of a defective measles virus genome derived from a diseased human brain. EMBO J 6(3), 681-8.

Cavazzana-Calvo, M., Hacein-Bey, S., de Saint Basile, G., Gross, F., Yvon, E., Nusbaum, P., Selz, F., Hue, C., Certain, S., Casanova, J. L., Bousso, P., Deist, F. L., and Fischer, A. (2000). Gene therapy of human severe combined immunodeficiency (SCID)-X1 disease. Science 288(5466), 669-72.

Chan, S. Y., Speck, R. F., Ma, M. C., and Goldsmith, M. A. (2000). Distinct mechanisms of entry by envelope glycoproteins of Marburg and Ebola (Zaire) viruses. J Virol 74(10), 4933-7.

Chesebro, B., Wehrly, K., and Maury, W. (1990). Differential expression in human and mouse cells of human immunodeficiency virus pseudotyped by murine retroviruses. J Virol 64(9), 4553-7.

Coffin, J. M. (1996). Retrovirus restriction revealed. Nature 382(6594), 762-3.

Counihan, M. E., Shay, D. K., Holman, R. C., Lowther, S. A., and Anderson, L. J. (2001). Human parainfluenza virus-associated hospitalizations among children less than five years of age in the United States. Pediatr Infect Dis J 20(7), 64653. 
Craft, W. W., Jr., and Dutch, R. E. (2005). Sequence motif upstream of the Hendra virus fusion protein cleavage site is not sufficient to promote efficient proteolytic processing. Virology 341(1), 130-40.

Cronin, J., Zhang, X. Y., and Reiser, J. (2005). Altering the tropism of lentiviral vectors through pseudotyping. Curr Gene Ther 5(4), 387-98.

Darai, G., Schwaier, A., Komitowski, D., and Munk, K. (1978). Experimental infection of Tupaia belangeri (tree shrews) with herpes simplex virus types 1 and $2 . J$ Infect Dis 137(3), 221-6.

De Palma, M., Venneri, M. A., and Naldini, L. (2003). In vivo targeting of tumor endothelial cells by systemic delivery of lentiviral vectors. Hum Gene Ther 14(12), 1193-206.

Desmaris, N., Bosch, A., Salaun, C., Petit, C., Prevost, M. C., Tordo, N., Perrin, P., Schwartz, O., de Rocquigny, H., and Heard, J. M. (2001). Production and neurotropism of lentivirus vectors pseudotyped with lyssavirus envelope glycoproteins. Mol Ther 4(2), 149-56.

Diederich, S., Moll, M., Klenk, H. D., and Maisner, A. (2005). The nipah virus fusion protein is cleaved within the endosomal compartment. J Biol Chem 280(33), 29899-903.

Dull, T., Zufferey, R., Kelly, M., Mandel, R. J., Nguyen, M., Trono, D., and Naldini, L. (1998). A third-generation lentivirus vector with a conditional packaging system. J Virol 72(11), 8463-71.

Eaton, B. T., Broder, C. C., Middleton, D., and Wang, L. F. (2006). Hendra and Nipah viruses: different and dangerous. Nat Rev Microbiol 4(1), 23-35.

Escors, D., and Breckpot, K. (2010). Lentiviral vectors in gene therapy: their current status and future potential. Arch Immunol Ther Exp (Warsz) 58(2), 107-19.

Frecha, C., Costa, C., Levy, C., Negre, D., Russell, S. J., Maisner, A., Salles, G., Peng, K. W., Cosset, F. L., and Verhoeyen, E. (2009). Efficient and stable transduction of resting $B$ lymphocytes and primary chronic lymphocyte leukemia cells using measles virus gp displaying lentiviral vectors. Blood 114(15), 3173-80.

Frecha, C., Costa, C., Negre, D., Gauthier, E., Russell, S. J., Cosset, F. L., and Verhoeyen, E. (2008). Stable transduction of quiescent T cells without induction of cycle progression by a novel lentiviral vector pseudotyped with measles virus glycoproteins. Blood 112(13), 4843-52.

Frecha, C., Levy, C., Cosset, F. L., and Verhoeyen, E. (2010). Advances in the field of lentivector-based transduction of $\mathrm{T}$ and $\mathrm{B}$ lymphocytes for gene therapy. Mol Ther 18(10), 1748-57.

Frecha, C., Levy, C., Costa, C., Negre, D., Amirache, F., Buckland, R., Russell, S. J., Cosset, F. L., and Verhoeyen, E. (2011). Measles virus glycoproteinpseudotyped lentiviral vector-mediated gene transfer into quiescent 
lymphocytes requires binding to both SLAM and CD46 entry receptors. $J$ Virol 85(12), 5975-85.

Freed, E. O. a. M., M.A. (2007). HIVs and Their Replication. 5 ed. In "Fields' Virology" (e. D.M. Knipe and P.M. Howley, Ed.), Vol. 5th edition, pp. pp. 21072186. Lippincott Williams \& Wilkins, Philadelphia.

Freiberg, A. N., Worthy, M. N., Lee, B., and Holbrook, M. R. (2010). Combined chloroquine and ribavirin treatment does not prevent death in a hamster model of Nipah and Hendra virus infection. J Gen Viro/ 91(Pt 3), 765-72.

Funke, S., Maisner, A., Muhlebach, M. D., Koehl, U., Grez, M., Cattaneo, R., Cichutek, K., and Buchholz, C. J. (2008a). Targeted cell entry of lentiviral vectors. Mol Ther 16(8), 1427-36.

Funke, S., Maisner, A., Mühlebach, M. D., Koehl, U., Grez, M., Cattaneo, R., Cichutek, K., and Buchholz, C. J. (2008b). Targeted cell entry of lentiviral vectors. Mol Ther 16(8), 1427-36.

Funke, S., Schneider, I. C., Glaser, S., Muhlebach, M. D., Moritz, T., Cattaneo, R., Cichutek, K., and Buchholz, C. J. (2009). Pseudotyping lentiviral vectors with the wild-type measles virus glycoproteins improves titer and selectivity. Gene Ther 16(5), 700-5.

Goldsmith, C. S., Whistler, T., Rollin, P. E., Ksiazek, T. G., Rota, P. A., Bellini, W. J., Daszak, P., Wong, K. T., Shieh, W. J., and Zaki, S. R. (2003). Elucidation of Nipah virus morphogenesis and replication using ultrastructural and molecular approaches. Virus Res 92(1), 89-98.

Goodbourn, S., and Randall, R. E. (2009). The regulation of type I interferon production by paramyxoviruses. J Interferon Cytokine Res 29(9), 539-47.

Green, N., Shinnick, T. M., Witte, O., Ponticelli, A., Sutcliffe, J. G., and Lerner, R. A. (1981). Sequence-specific antibodies show that maturation of Moloney leukemia virus envelope polyprotein involves removal of a $\mathrm{COOH}$-terminal peptide. Proc Natl Acad Sci U S A 78(10), 6023-7.

Harrison, M. S., Sakaguchi, T., and Schmitt, A. P. (2010). Paramyxovirus assembly and budding: building particles that transmit infections. Int J Biochem Cell Biol 42(9), 1416-29.

Hematti, P., Hong, B. K., Ferguson, C., Adler, R., Hanawa, H., Sellers, S., Holt, I. E., Eckfeldt, C. E., Sharma, Y., Schmidt, M., von Kalle, C., Persons, D. A., Billings, E. M., Verfaillie, C. M., Nienhuis, A. W., Wolfsberg, T. G., Dunbar, C. E., and Calmels, B. (2004). Distinct genomic integration of MLV and SIV vectors in primate hematopoietic stem and progenitor cells. PLoS Biol 2(12), e423.

Henderson, L. E., Sowder, R., Copeland, T. D., Smythers, G., and Oroszlan, S. (1984). Quantitative separation of murine leukemia virus proteins by reversedphase high-pressure liquid chromatography reveals newly described gag and env cleavage products. J Virol 52(2), 492-500. 
Hernandez, L. D., Hoffman, L. R., Wolfsberg, T. G., and White, J. M. (1996). Viruscell and cell-cell fusion. Annu Rev Cell Dev Biol 12, 627-61.

Hoggard, P. G., and Owen, A. (2003). The mechanisms that control intracellular penetration of the HIV protease inhibitors. J Antimicrob Chemother 51(3), 4936.

Hu, W. S., and Pathak, V. K. (2000). Design of retroviral vectors and helper cells for gene therapy. Pharmacol Rev 52(4), 493-511.

Janssens, W., Chuah, M. K., Naldini, L., Follenzi, A., Collen, D., Saint-Remy, J. M., and VandenDriessche, T. (2003). Efficiency of onco-retroviral and lentiviral gene transfer into primary mouse and human B-lymphocytes is pseudotype dependent. Hum Gene Ther 14(3), 263-76.

Kaiser, J. (2003). Gene therapy. Seeking the cause of induced leukemias in X-SCID trial. Science 299(5606), 495.

Katz, R. A., and Skalka, A. M. (1994). The retroviral enzymes. Annu Rev Biochem 63, 133-73.

Klenk, H. D., and Garten, W. (1994). Host cell proteases controlling virus pathogenicity. Trends Microbiol 2(2), 39-43.

Kobayashi, M., lida, A., Ueda, Y., and Hasegawa, M. (2003). Pseudotyped lentivirus vectors derived from simian immunodeficiency virus SIVagm with envelope glycoproteins from paramyxovirus. J Virol 77(4), 2607-14.

Kobinger, G. P., Weiner, D. J., Yu, Q. C., and Wilson, J. M. (2001). Filoviruspseudotyped lentiviral vector can efficiently and stably transduce airway epithelia in vivo. Nat Biotechnol 19(3), 225-30.

Korin, Y. D., and Zack, J. A. (1998). Progression to the G1b phase of the cell cycle is required for completion of human immunodeficiency virus type 1 reverse transcription in T cells. J Virol 72(4), 3161-8.

Laemmli, U. K. (1970). Cleavage of structural proteins during the assembly of the head of bacteriophage T4. Nature 227(5259), 680-5.

Lamb, H. K., Parks, G.D. (2007). Paramyxoviridae: The Viruses and Their Replication. In "Fields' Virology" (e. D.M. Knipe and P.M. Howley, Ed.), Vol. 5th edition, pp. pp. 1449-1496. Lippincott Williams \& Wilkins, Philadelphia.

Lamb, R. A., and Jardetzky, T. S. (2007). Structural basis of viral invasion: lessons from paramyxovirus F. Curr Opin Struct Biol 17(4), 427-36.

Lee, B., and Ataman, Z. A. (2011). Modes of paramyxovirus fusion: a Henipavirus perspective. Trends Microbiol 19(8), 389-99.

Levine, F., and Friedmann, T. (1991). Gene therapy techniques. Curr Opin Biotechnol 2(6), 840-4. 
Mann, R., Mulligan, R. C., and Baltimore, D. (1983). Construction of a retrovirus packaging mutant and its use to produce helper-free defective retrovirus. Cell 33(1), 153-9.

Matrai, J., Chuah, M. K., and VandenDriessche, T. (2010). Recent advances in lentiviral vector development and applications. Mol Ther 18(3), 477-90.

Meulendyke, K. A., Wurth, M. A., McCann, R. O., and Dutch, R. E. (2005). Endocytosis plays a critical role in proteolytic processing of the Hendra virus fusion protein. J Virol 79(20), 12643-9.

Miest, T. S., Yaiw, K. C., Frenzke, M., Lampe, J., Hudacek, A. W., Springfeld, C., von Messling, V., Ungerechts, G., and Cattaneo, R. (2011). Envelope-chimeric Entry-targeted Measles Virus Escapes Neutralization and Achieves Oncolysis. Mol Ther.

Miyoshi, H., Takahashi, M., Gage, F. H., and Verma, I. M. (1997). Stable and efficient gene transfer into the retina using an HIV-based lentiviral vector. Proc Natl Acad Sci U S A 94(19), 10319-23.

Modlich, U., Navarro, S., Zychlinski, D., Maetzig, T., Knoess, S., Brugman, M. H., Schambach, A., Charrier, S., Galy, A., Thrasher, A. J., Bueren, J., and Baum, C. (2009). Insertional transformation of hematopoietic cells by self-inactivating lentiviral and gammaretroviral vectors. Mol Ther 17(11), 1919-28.

Moll, M., Klenk, H. D., and Maisner, A. (2002). Importance of the cytoplasmic tails of the measles virus glycoproteins for fusogenic activity and the generation of recombinant measles viruses. J Virol 76(14), 7174-86.

Morizono, K., Xie, Y., Ringpis, G. E., Johnson, M., Nassanian, H., Lee, B., Wu, L., and Chen, I. S. (2005). Lentiviral vector retargeting to P-glycoprotein on metastatic melanoma through intravenous injection. Nat Med 11(3), 346-52.

Mossadegh, N., Gissmann, L., Muller, M., Zentgraf, H., Alonso, A., and Tomakidi, P. (2004). Codon optimization of the human papillomavirus 11 (HPV 11) L1 gene leads to increased gene expression and formation of virus-like particles in mammalian epithelial cells. Virology 326(1), 57-66.

Munch, R. C., Muhlebach, M. D., Schaser, T., Kneissl, S., Jost, C., Pluckthun, A., Cichutek, K., and Buchholz, C. J. (2011). DARPins: an efficient targeting domain for lentiviral vectors. Mol Ther 19(4), 686-93.

Murakami, T., and Freed, E. O. (2000a). Genetic evidence for an interaction between human immunodeficiency virus type 1 matrix and alpha-helix 2 of the gp41 cytoplasmic tail. J Virol 74(8), 3548-54.

Murakami, T., and Freed, E. O. (2000b). The long cytoplasmic tail of gp41 is required in a cell type-dependent manner for HIV-1 envelope glycoprotein incorporation into virions. Proc Natl Acad Sci U S A 97(1), 343-8. 
Naim, H. Y., and Roth, M. G. (1994). Characteristics of the internalization signal in the Y543 influenza virus hemagglutinin suggest a model for recognition of internalization signals containing tyrosine. J Biol Chem 269(6), 3928-33.

Nakamura, T., Peng, K. W., Harvey, M., Greiner, S., Lorimer, I. A., James, C. D., and Russell, S. J. (2005). Rescue and propagation of fully retargeted oncolytic measles viruses. Nat Biotechnol 23(2), 209-14.

Naldini, L., Blomer, U., Gallay, P., Ory, D., Mulligan, R., Gage, F. H., Verma, I. M., and Trono, D. (1996). In vivo gene delivery and stable transduction of nondividing cells by a lentiviral vector. Science 272(5259), 263-7.

Navaratnarajah, C. K., Leonard, V. H., and Cattaneo, R. (2009). Measles virus glycoprotein complex assembly, receptor attachment, and cell entry. Curr Top Microbiol Immunol 329, 59-76.

Noyce, R. S., Bondre, D. G., Ha, M. N., Lin, L. T., Sisson, G., Tsao, M. S., and Richardson, C. D. (2011). Tumor Cell Marker PVRL4 (Nectin 4) Is an Epithelial Cell Receptor for Measles Virus. PLoS Pathog 7(8), e1002240.

Oertel, M., Rosencrantz, R., Chen, Y. Q., Thota, P. N., Sandhu, J. S., Dabeva, M. D., Pacchia, A. L., Adelson, M. E., Dougherty, J. P., and Shafritz, D. A. (2003). Repopulation of rat liver by fetal hepatoblasts and adult hepatocytes transduced ex vivo with lentiviral vectors. Hepatology 37(5), 994-1005.

Otto, H. H., and Schirmeister, T. (1997). Cysteine Proteases and Their Inhibitors. Chem Rev 97(1), 133-172.

Pager, C. T., Craft, W. W., Jr., Patch, J., and Dutch, R. E. (2006). A mature and fusogenic form of the Nipah virus fusion protein requires proteolytic processing by cathepsin L. Virology 346(2), 251-7.

Pager, C. T., and Dutch, R. E. (2005). Cathepsin L is involved in proteolytic processing of the Hendra virus fusion protein. J Virol 79(20), 12714-20.

Pickl, W. F., Pimentel-Muinos, F. X., and Seed, B. (2001). Lipid rafts and pseudotyping. J Virol 75(15), 7175-83.

Plumet, S., Duprex, W. P., and Gerlier, D. (2005). Dynamics of viral RNA synthesis during measles virus infection. J Virol 79(11), 6900-8.

Pluta, K., and Kacprzak, M. M. (2009). Use of HIV as a gene transfer vector. Acta Biochim Pol 56(4), 531-95.

Porotto, M., Orefice, G., Yokoyama, C. C., Mungall, B. A., Realubit, R., Sganga, M. L., Aljofan, M., Whitt, M., Glickman, F., and Moscona, A. (2009). Simulating henipavirus multicycle replication in a screening assay leads to identification of a promising candidate for therapy. J Virol 83(10), 5148-55.

Ramachandran, A., and Horvath, C. M. (2009). Paramyxovirus disruption of interferon signal transduction: STATus report. J Interferon Cytokine Res 29(9), 531-7. 
Reddy, V. Y., Zhang, Q. Y., and Weiss, S. J. (1995). Pericellular mobilization of the tissue-destructive cysteine proteinases, cathepsins $B$, L, and $S$, by human monocyte-derived macrophages. Proc Natl Acad Sci U S A 92(9), 3849-53.

Reiser, J., Harmison, G., Kluepfel-Stahl, S., Brady, R. O., Karlsson, S., and Schubert, M. (1996). Transduction of nondividing cells using pseudotyped defective high-titer HIV type 1 particles. Proc Natl Acad Sci U S A 93(26), 15266-71.

Sakalian, M., and Hunter, E. (1998). Molecular events in the assembly of retrovirus particles. Adv Exp Med Biol 440, 329-39.

Sandrin, V., Muriaux, D., Darlix, J. L., and Cosset, F. L. (2004). Intracellular trafficking of $\mathrm{Gag}$ and Env proteins and their interactions modulate pseudotyping of retroviruses. J Virol 78(13), 7153-64.

Schneider, I. C., Eckhardt, M., Brynza, J., Collins, M. K., Cichutek, K., and Buchholz, C. J. (2011). Escape from R-peptide deletion in a gamma-retrovirus. Virology 418(2), 85-92.

Schneider, U., Bullough, F., Vongpunsawad, S., Russell, S. J., and Cattaneo, R. (2000). Recombinant measles viruses efficiently entering cells through targeted receptors. J Virol 74(21), 9928-36.

Schnierle, B. S., Stitz, J., Bosch, V., Nocken, F., Merget-Millitzer, H., Engelstadter, M., Kurth, R., Groner, B., and Cichutek, K. (1997). Pseudotyping of murine leukemia virus with the envelope glycoproteins of HIV generates a retroviral vector with specificity of infection for CD4-expressing cells. Proc Natl Acad Sci U S A 94(16), 8640-5.

Serafini, M., Naldini, L., and Introna, M. (2004). Molecular evidence of inefficient transduction of proliferating human B lymphocytes by VSV-pseudotyped HIV1-derived lentivectors. Virology 325(2), 413-24.

Spector, D. H., Wade, E., Wright, D. A., Koval, V., Clark, C., Jaquish, D., and Spector, S. A. (1990). Human immunodeficiency virus pseudotypes with expanded cellular and species tropism. J Virol 64(5), 2298-308.

Springfeld, C., von Messling, V., Tidona, C. A., Darai, G., and Cattaneo, R. (2005). Envelope targeting: hemagglutinin attachment specificity rather than fusion protein cleavage-activation restricts Tupaia paramyxovirus tropism. J Virol 79(16), 10155-63.

Sukhova, G. K., Shi, G. P., Simon, D. I., Chapman, H. A., and Libby, P. (1998). Expression of the elastolytic cathepsins $\mathrm{S}$ and $\mathrm{K}$ in human atheroma and regulation of their production in smooth muscle cells. J Clin Invest 102(3), 57683.

Swanstrom, R., and Wills, J. W. (1997). Synthesis, Assembly, and Processing of Viral Proteins.

Szecsi, J., Drury, R., Josserand, V., Grange, M. P., Boson, B., Hartl, I., Schneider, R., Buchholz, C. J., Coll, J. L., Russell, S. J., Cosset, F. L., and Verhoeyen, E. 
(2006). Targeted retroviral vectors displaying a cleavage site-engineered hemagglutinin (HA) through HA-protease interactions. Mol Ther 14(5), 735-44.

Tidona, C. A. (1999). Development and application of methods for efficient characterization of new RNA viruses in example of Tupaia paramyxovirus. PhD Thesis, Heidelberg University, Ruperto Carola.

Tidona, C. A., Kurz, H. W., Gelderblom, H. R., and Darai, G. (1999). Isolation and molecular characterization of a novel cytopathogenic paramyxovirus from tree shrews. Virology 258(2), 425-34.

Turk, B., Turk, D., and Turk, V. (2000). Lysosomal cysteine proteases: more than scavengers. Biochim Biophys Acta 1477(1-2), 98-111.

Unutmaz, D., KewalRamani, V. N., Marmon, S., and Littman, D. R. (1999). Cytokine signals are sufficient for HIV-1 infection of resting human T lymphocytes. $J$ Exp Med 189(11), 1735-46.

VandenDriessche, T., Thorrez, L., Naldini, L., Follenzi, A., Moons, L., Berneman, Z., Collen, D., and Chuah, M. K. (2002). Lentiviral vectors containing the human immunodeficiency virus type-1 central polypurine tract can efficiently transduce nondividing hepatocytes and antigen-presenting cells in vivo. Blood 100(3), 813-22.

Verma, I. M., and Weitzman, M. D. (2005). Gene therapy: twenty-first century medicine. Annu Rev Biochem 74, 711-38.

Vogt, V. M. (1997). Retroviral Virions and Genomes.

Vogt, V. M., and Simon, M. N. (1999). Mass determination of rous sarcoma virus virions by scanning transmission electron microscopy. J Virol 73(8), 7050-5.

von Messling, V., Milosevic, D., Devaux, P., and Cattaneo, R. (2004). Canine distemper virus and measles virus fusion glycoprotein trimers: partial membrane-proximal ectodomain cleavage enhances function. J Virol 78(15), 7894-903.

Vongpunsawad, S., Oezgun, N., Braun, W., and Cattaneo, R. (2004). Selectively receptor-blind measles viruses: Identification of residues necessary for SLAMor CD46-induced fusion and their localization on a new hemagglutinin structural model. J Virol 78(1), 302-13.

Waehler, R., Russell, S. J., and Curiel, D. T. (2007). Engineering targeted viral vectors for gene therapy. Nat Rev Genet 8(8), 573-87.

Watanabe, S., and Temin, H. M. (1982). Encapsidation sequences for spleen necrosis virus, an avian retrovirus, are between the $5^{\prime}$ long terminal repeat and the start of the gag gene. Proc Natl Acad Sci U S A 79(19), 5986-90.

Watson, D. J., Kobinger, G. P., Passini, M. A., Wilson, J. M., and Wolfe, J. H. (2002). Targeted transduction patterns in the mouse brain by lentivirus vectors pseudotyped with VSV, Ebola, Mokola, LCMV, or MuLV envelope proteins. Mol Ther 5(5 Pt 1), 528-37. 
Whelan, S. P., Barr, J. N., and Wertz, G. W. (2004). Transcription and replication of nonsegmented negative-strand RNA viruses. Curr Top Microbiol Immunol 283, $61-119$.

Xie, Z. C., Riezu-Boj, J. I., Lasarte, J. J., Guillen, J., Su, J. H., Civeira, M. P., and Prieto, J. (1998). Transmission of hepatitis $C$ virus infection to tree shrews. Virology 244(2), 513-20.

Yoder, A., Yu, D., Dong, L., Iyer, S. R., Xu, X., Kelly, J., Liu, J., Wang, W., Vorster, P. J., Agulto, L., Stephany, D. A., Cooper, J. N., Marsh, J. W., and Wu, Y. (2008). HIV envelope-CXCR4 signaling activates cofilin to overcome cortical actin restriction in resting CD4 T cells. Cell 134(5), 782-92.

Zhou, Q., Schneider, I. C., Gallet, M., Kneissl, S., and Buchholz, C. J. (2011). Resting lymphocyte transduction with measles virus glycoprotein pseudotyped lentiviral vectors relies on CD46 and SLAM. Virology 413(2), 149-52.

Zhu, Z. H., Chen, S. S., and Huang, A. S. (1990). Phenotypic mixing between human immunodeficiency virus and vesicular stomatitis virus or herpes simplex virus. J Acquir Immune Defic Syndr 3(3), 215-9.

Zufferey, R., Nagy, D., Mandel, R. J., Naldini, L., and Trono, D. (1997). Multiply attenuated lentiviral vector achieves efficient gene delivery in vivo. Nat Biotechnol 15(9), 871-5. 